\title{
Lefschetz fibrations and symplectic homology
}

\author{
MARK MCLEAN
}

\begin{abstract}
We show that for each $k>3$ there are infinitely many finite type Stein manifolds diffeomorphic to Euclidean space $\mathbb{R}^{2 k}$ which are pairwise distinct as symplectic manifolds.
\end{abstract}

$53 \mathrm{D} 35 ; 53 \mathrm{D} 40$

\section{Introduction}

This paper is about the symplectic topology of Stein manifolds. If we have a symplectic manifold $(V, \omega)$, then we say it carries a Stein structure if there exists a complex structure $J$ and an exhausting (ie proper and bounded from below) plurisubharmonic function $\phi: V \rightarrow \mathbb{R}$ such that $\omega=-d d^{c} \phi$, where $d^{c}$ is defined by $d^{c}(a)(X):=$ $d a(J X)$. The triple $(V, J, \phi)$ is called a Stein manifold. We say that a Stein manifold is of finite type if $\phi$ only has finitely many critical points, each of which is nondegenerate. We define an equivalence relation $\sim$ on Stein manifolds by: $A \sim B$ if there exists a sequence of Stein manifolds $F_{0}, F_{1}, \ldots, F_{n}$ such that:

(1) $F_{0}=A$ and $F_{n}=B$.

(2) $F_{i}$ is either symplectomorphic or Stein deformation equivalent to $F_{i+1}$. (Stein deformation is defined later in Definition 2.7.)

The aim of this paper is the following theorem:

Theorem 1.1 Let $k \geq 4$. There exists a family of finite type Stein manifolds $X_{i}$ diffeomorphic to $\mathbb{R}^{2 k}$ indexed by $i \in \mathbb{N}$ such that

$$
i \neq j \Rightarrow X_{i} \nsim X_{j}
$$

We also have the following corollary:

Corollary 1.2 Let $M$ be a compact manifold of dimension 4 or higher. There exists a family of finite type Stein manifolds $X_{i}^{M}$ diffeomorphic to $T^{*} M$ indexed by $i \in \mathbb{N}$ such that

$$
i \neq j \Rightarrow X_{i}^{M} \nsim X_{j}^{M} .
$$


We will prove this at the end of this introduction. The results of Paul Seidel and Ivan Smith [27] show that there exists a finite type Stein manifold diffeomorphic to $\mathbb{R}^{4 k}$ but not symplectomorphic to $\mathbb{R}^{4 k}, k \geq 2$. They show this by constructing an affine variety which has a Lagrangian torus which cannot be moved off itself by a Hamiltonian isotopy. In fact they show that none of these Stein manifolds can be embedded in a subcritical Stein manifold. Also, they have an argument (explained by Seidel [25]) that shows that the symplectic homology groups of these varieties are nontrivial. In this paper we strengthen this result in two ways:

(1) We give examples in all even dimensions $\geq 8$ (not just in dimension $4 k$ where $k \geq 2$ ).

(2) We show there are countably many pairwise distinct examples in each of these dimensions.

(1) is straightforward but (2) is much harder and involves various new ideas. We show in Corollary 10.4 that these manifolds cannot be embedded in a subcritical Stein manifold.

There is no analogue of our result in dimension 4 because any finite type Stein manifold diffeomorphic to $\mathbb{R}^{4}$ is symplectomorphic to $\mathbb{R}^{4}$ (see the introduction to [27]). Having said that, Gompf [16] constructs uncountably many nonfinite type Stein manifolds which are homeomorphic to $\mathbb{R}^{4}$, but are pairwise not diffeomorphic to each other. We hope to address the question of whether Theorem 1.1 holds in dimension 6 , and whether we can distinguish the contact boundaries of these manifolds up to contactomorphism, in future work.

We will now construct an example of a family of Stein manifolds $\left(X_{n}\right)_{n \in \mathbb{N}}$ as in Theorem 1.1 in dimension 8 . Let $V:=\left\{x^{7}+y^{2}+z^{2}+w^{2}=0\right\} \subset \mathbb{C}^{4}$ and consider a smooth point, say $p:=(0,0,1, i) \in V$. Let $H$ be the blowup of $\mathbb{C}^{4}$ at $p$. Then $X:=H \backslash \widetilde{V}$ is a Stein manifold where $\tilde{V}$ is the proper transform of $V$. The variety $X$ is called the Kaliman modification of $\left(\mathbb{C}^{4}, V, p\right)$ (see Kaliman [18]). We will think of this modification in two stages:

(1) Cut out the hypersurface $V$ in $\mathbb{C}^{4}$ to get $Z:=\mathbb{C}^{4} \backslash V$.

(2) Blow up $Z$ at infinity to get $X$.

Operation (2) attaches a 2-handle along a knot which is transverse to the contact structure. All our Stein manifolds in this family will be constructed from $X$. If we have two Stein manifolds $A$ and $B$, then it is possible to construct their end connected sum $A \#_{e} B$ (see Theorem 2.10). Roughly what we do here is join $A$ and $B$ with a 1 -handle, and then extend the Stein structure over this handle. Finally, $X_{n}:=\#_{i=1}^{n} X$ is our family of Stein manifolds. 


\subsection{Sketch of the proof of the main theorem}

We will now give an outline of the proof. We will only consider the examples $X_{n}$ in dimension 8 constructed above, as the higher dimensional examples are similar. For each Stein manifold $Y$ with a trivialisation of the canonical bundle, we have an integer graded commutative $\mathbb{Z} / 2 \mathbb{Z}$ algebra $\mathrm{SH}_{n+*}(Y)$ where $\mathrm{SH}_{*}(Y)$ is called symplectic homology ${ }^{1}$. The reason why we write $\mathrm{SH}_{n+*}(Y)$ instead of $\mathrm{SH}_{*}(Y)$ is because we want the unit to be in degree 0 and not in degree $n$. If $Y_{1}$ and $Y_{2}$ are Stein manifolds with $H_{1}\left(Y_{1}\right)=H_{1}\left(Y_{2}\right)=0$ and $Y_{1} \sim Y_{2}$, then $\operatorname{SH}_{*}\left(Y_{1}\right)=\operatorname{SH}_{*}\left(Y_{2}\right)$ (see Seidel [25, Section 7]). The reason why we need $H_{1}\left(Y_{1}\right)=H_{1}\left(Y_{2}\right)=0$ is because symplectic homology is only known to be invariant up to exact symplectomorphism. For each Stein manifold $Y$, we can define another invariant $i(Y)$ which is the number of idempotents of $\mathrm{SH}_{*}(Y)$ (this invariant might be infinite). Hence all we need to do is show that for $i \neq j, i\left(X_{i}\right) \neq i\left(X_{j}\right)$. The next fact we need is that for any two Stein manifolds $Y_{1}$ and $Y_{2}, \mathrm{SH}_{*}\left(Y_{1} \#_{e} Y_{2}\right)=\mathrm{SH}_{*}\left(Y_{1}\right) \times \mathrm{SH}_{*}\left(Y_{2}\right)$. This means that $\mathrm{SH}_{*}\left(X_{n}\right)=\prod_{i=1}^{n} \mathrm{SH}_{*}(X)$, and hence $i\left(X_{n}\right)=i(X)^{n}$. So all we need to do is show that $1<i(X)<\infty$. If $\mathrm{SH}_{*}(X) \neq 0, i(X)>1$ since we have 0 and 1 ; but since $\mathrm{SH}_{*}(X)$ can a priori be infinite dimensional in each degree, finiteness of $i(X)$ is much harder. Most of the work in this paper involves proving $i(X)<\infty$.

For any Stein manifold $Y, \mathrm{SH}_{*}(Y)$ is $\mathbb{Z}$-graded by the Conley-Zehnder index taken with negative sign. The group $\mathrm{SH}_{*}(Y)$ has a ring structure making $\mathrm{SH}_{n+*}(Y)$ into a $\mathbb{Z} / 2 \mathbb{Z}$-graded algebra. This ring is also a $H_{1}(Y)$-graded algebra. This means that as a vector space, it is of the form $\bigoplus_{i \in H_{1}(Y)} R_{i}$ and if $x \in R_{a}$ and $y \in R_{b}$ then their product $x y$ is in $R_{a+b}$. Hence idempotents in $\mathrm{SH}_{n+*}(Y)$ are contained in $\mathrm{SH}_{n}(Y)$ and are a linear combination of elements with grading in the torsion part of $H_{1}(Y)$ (see Lemma 7.6). The problem is that $H_{1}(X)=0$. In order to find out which elements of $\mathrm{SH}_{n+*}(X)$ are idempotents, we will show that $\mathrm{SH}_{*}(X)$ is isomorphic as a ring to $\mathrm{SH}_{*}(Z)$ where $Z=\mathbb{C}^{4} \backslash V$ was defined above. Because $Z$ is so much simpler than $X$ and $H_{1}(Z) \neq 0$, it is possible by a direct calculation to show that $\mathrm{SH}_{n+*}(Z)$ has finitely many idempotents.

Proving that $\mathrm{SH}_{*}(X) \cong \mathrm{SH}_{*}(Z)$ relies on the following theorem. This theorem is the heart of the proof. We let $E^{\prime} \rightarrow \mathbb{C}, E^{\prime \prime} \rightarrow \mathbb{C}$ be Lefschetz fibrations, and $F^{\prime}$ (resp. $F^{\prime \prime}$ ) be smooth fibres of $E^{\prime}$ (resp. $E^{\prime \prime}$ ). Let $F^{\prime}$ and $F^{\prime \prime}$ be Stein domains with $F^{\prime \prime}$ a holomorphic and symplectic submanifold of $F^{\prime}$.

Theorem 1.3 Suppose $E^{\prime}$ and $E^{\prime \prime}$ satisfy the following properties:

(1) $E^{\prime \prime}$ is a subfibration of $E^{\prime}$.

\footnotetext{
${ }^{1}$ With our convention, the pair-of-pants product makes $\mathrm{SH}_{*}$ (and not $\mathrm{SH}^{*}$ ) a unital ring.
} 
(2) The support of all the monodromy maps of $E^{\prime}$ are contained in the interior of $E^{\prime \prime}$.

(3) Any holomorphic curve in $F^{\prime}$ with boundary inside $F^{\prime \prime}$ must be contained in $F^{\prime \prime}$.

Then $\mathrm{SH}_{*}\left(E^{\prime}\right) \cong \mathrm{SH}_{*}\left(E^{\prime \prime}\right)$.

Remark 1 There exist Lefschetz fibrations $E^{\prime}, E^{\prime \prime}$ with the above properties such that as convex symplectic manifolds, $E^{\prime}$ (resp. $E^{\prime \prime}$ ) is convex deformation equivalent to $X$ (resp. $Z$ ). This is because we can choose an algebraic Lefschetz fibration on $Z$ where the closures of all the fibres pass through $p$. Then blowing up $Z$ at infinity (operation (2) of the Kaliman modification) is the same as blowing up each fibre at infinity and keeping the same monodromy. Hence $\mathrm{SH}_{*}(X) \cong \mathrm{SH}_{*}(Z)$.

Remark 2 Given varieties $X$ and $Z$ in dimension 4 such that $X$ is obtained from $Z$ by blowing up at infinity, there are Lefschetz fibrations $E^{\prime}, E^{\prime \prime}$ satisfying properties (1) and (2) such that as convex symplectic manifolds, $E^{\prime}$ (resp. $E^{\prime \prime}$ ) is convex deformation equivalent to $X$ (resp. $Z$ ). These do not satisfy property (3) because $E^{\prime}$ is obtained from $E^{\prime \prime}$ by filling in a boundary component of the fibres with a disc.

We will prove Theorem 1.3 in two stages. In stage (i), we construct a $\mathbb{Z} / 2 \mathbb{Z}$-graded algebra $\mathrm{SH}_{*}^{\text {lef }}(E)$ related to a Lefschetz fibration $E$ and show it is equal to symplectic homology. This is covered in Sections 4 and 5.1. In stage (ii), we prove that $\operatorname{SH}_{*}^{\text {lef }}\left(E^{\prime}\right) \cong$ $\mathrm{SH}_{*}^{\text {lef }}\left(E^{\prime \prime}\right)$. This is covered in Section 6. In a little more detail:

(i) Let $F$ be a smooth fibre of $E$ and $\mathbb{D}$ a disc in $\mathbb{C}$. In Section 5, we show (roughly) that the chain complex $C$ for $\mathrm{SH}_{*}(E)$ is generated by:

(1) Critical points of some Morse function on $E$.

(2) Two copies of fixed points of iterates of the monodromy map around a large circle.

(3) Pairs $(\Gamma, \gamma)$ where $\Gamma$ is a Reeb orbit on the boundary of $F$ and $\gamma$ is either a Reeb orbit of $\partial \mathbb{D}$ or a fixed point in the interior of $\mathbb{D}$.

This is done in almost exactly the same way as the proof of the Künneth formula for symplectic homology by Oancea [21]. The differential as usual involves counting cylinders connecting the orbits and satisfying the perturbed Cauchy-Riemann equations. The orbits in (1) and (2) actually form a subcomplex $C^{\text {lef }}$, and we define Lefschetz symplectic homology $\mathrm{SH}_{*}^{\text {lef }}(E)$ to be the homology of this subcomplex. Let $\Phi: \mathrm{SH}_{k}^{\text {lef }}(E) \rightarrow \mathrm{SH}_{k}(E)$ be the map corresponding to the inclusion $C^{\text {lef }} \hookrightarrow C$. We 
need to show that $\Phi$ is an isomorphism. In order to do this we will show that the elements of $C$ in (3) have very high index. The orbits $\gamma$ in (3) actually correspond to orbits of some Hamiltonian on the complex plane $\mathbb{C}$. We can ensure that this Hamiltonian has orbits of index as large as we like. In particular, we can assume that the index of $\gamma$ is so large that the index of the pair $(\gamma, \Gamma)$ is greater than any number we like (because the index of $(\gamma, \Gamma)$ is the sum $\operatorname{index}(\gamma)+\operatorname{index}(\Gamma))$. This means for any $k \in \mathbb{Z}$, the map $\mathrm{SH}_{k}^{\text {lef }}(E) \rightarrow \mathrm{SH}_{k}(E)$ is an isomorphism if we ensure the indices of the orbits $(\gamma, \Gamma)$ are all greater than $k+1$. Hence $\operatorname{SH}_{*}(E) \cong \operatorname{SH}_{*}^{\text {lef }}(E)$.

(ii) Let $C^{\prime}$ (resp. $C^{\prime \prime}$ ) be the chain complex for the group $\mathrm{SH}_{*}^{\text {lef }}\left(E^{\prime}\right)\left(\operatorname{resp} . \mathrm{SH}_{*}^{\text {lef }}\left(E^{\prime \prime}\right)\right)$. The fibration $E^{\prime} \backslash E^{\prime \prime}$ is a trivial fibration $\mathbb{D} \times W$. We have a short exact sequence $0 \rightarrow B \rightarrow C^{\prime} \rightarrow C^{\prime \prime} \rightarrow 0$ where $B$ is generated by orbits of the form $(\gamma, \Gamma)$ in $\mathbb{D} \times W$. The orbit $\Gamma$ is a critical point of some Morse function on $W$ and $\gamma$ is either a Reeb orbit of $\partial \mathbb{D}$ or a fixed point in the interior of $\mathbb{D}$. In this case the homology of the chain complex $B$ is actually a product $\mathrm{SH}_{*}(\mathbb{D}) \otimes X$ where $X$ is the relative cohomology group $H^{n-*}\left(F^{\prime}, F^{\prime \prime}\right)$. Because $\mathrm{SH}_{*}(\mathbb{D})=0$, we have that $H_{*}(B)=0$ which gives us our isomorphism between $\operatorname{SH}_{*}^{\text {lef }}\left(E^{\prime}\right)$ and $\operatorname{SH}_{*}^{\text {lef }}\left(E^{\prime \prime}\right)$. Property (3) in Theorem 1.3 is needed here to ensure that the above exact sequence exists. If we didn't have this property, then there would be some spectral sequence from $\operatorname{SH}_{*}^{\text {lef }}\left(E^{\prime \prime}\right)$ (with an extra grading coming from the $H_{1}\left(E^{\prime \prime}\right)$ classes of these orbits) to $\mathrm{SH}_{*}^{\text {lef }}\left(E^{\prime}\right)$.

Lefschetz symplectic homology was partially inspired by Paul Seidel's Hochshild homology conjectures [23], which also relate symplectic homology to Lefschetz fibrations. His conjectures would in particular prove Theorem 1.3.

Proof of Corollary 1.2 There is a standard Stein structure on $T^{*} M$ such that $\mathrm{SH}_{0}(M)$ is a nontrivial finite dimensional $\mathbb{Z} / 2 \mathbb{Z}$ vector space. By Lemma 7.6 this means that $i\left(T^{*} M\right)<\infty$. Also $0 \in \mathrm{SH}_{*}\left(T^{*} M\right)$ is an idempotent which means that $0<i\left(T^{*} M\right)$. We let $X_{i}$ be defined as in the proof of the main Theorem 1.1. We define

$$
X_{i}^{M}:=T^{*} M \#_{e} X_{i}
$$

Then $i\left(X_{i}^{M}\right)=i\left(T^{*} M\right) i\left(X_{i}\right)$. These numbers are all different as $0<i\left(T^{*} M\right)<\infty$ and $i\left(X_{i}\right) \neq i\left(X_{j}\right)$ for $i \neq j$.

Acknowledgements I would like to thank my supervisor Ivan Smith for checking this paper and for giving many useful suggestions. I would also like to thank Paul Seidel, Kai Cieliebak, Alexandru Oancea, Frédéric Bourgeois, Dominic Joyce, Burt Totaro, Jonny Evans and Jack Waldron for giving useful comments. 


\subsection{Notation}

Throughout this paper we use the following notation:

(1) $M, M^{\prime}, M^{\prime \prime}, \ldots$ are manifolds (with or without boundary).

(2) $\partial M$ is the boundary of $M$.

(3) $(E, \pi),\left(E^{\prime}, \pi^{\prime}\right),\left(E^{\prime \prime}, \pi^{\prime \prime}\right)$ are exact Lefschetz fibrations (See Definition 2.12).

(4) If we have some data $X$ associated to $M$ (resp. $E$ ), then $X, X^{\prime}, X^{\prime \prime}, \ldots$ are data associated to $M, M^{\prime}, M^{\prime \prime}, \ldots$ (resp. $E, E^{\prime}, E^{\prime \prime}, \ldots$ ). For instance $\partial M^{\prime \prime}$ is the boundary of $M^{\prime \prime}$.

(5) $\omega$ is a symplectic form on $M$ or $E$.

(6) $\theta$ is a $1-$ form such that $d \theta=\omega$.

(7) $(M, \theta)$ is an exact symplectic manifold.

(8) $J$ is an almost complex structure compatible with $\omega$.

(9) If $(M, \theta)$ is a compact convex symplectic manifold (see Definition 2.1), then $(\widehat{M}, \theta)$ is the completion of $(M, \theta)$ (Lemma 2.4). Similarly by Definition 2.16, $(E, \pi)$ can be completed to $(\widehat{E}, \pi)$ (we leave $\pi$ and $\theta$ as they are by abuse of notation).

(10) $\left(M, \theta_{t}\right)$ is a convex symplectic or Stein deformation.

(11) $F$ will denote a smooth fibre of $(E, \pi)$.

(12) If we have some subset $A$ of a topological space, then we will let $\operatorname{nhd}(A)$ be some open neighbourhood of $A$.

\section{Background}

\subsection{Stein manifolds}

We will define Stein manifolds as in Seidel and Smith [27]. We let $M$ be a manifold and $\theta$ a 1 -form where $\omega:=d \theta$ is a symplectic form.

Definition 2.1 $(M, \theta)$ is called a compact convex symplectic manifold if $M$ is a compact manifold with boundary and the $\omega$-dual of $\theta$ is transverse to $\partial M$ and pointing outwards. A compact convex symplectic deformation is a family of compact convex symplectic manifolds $\left(M, \theta_{t}\right)$ parameterized by $t \in[0,1]$. We will let $\lambda$ be the vector field which is $\omega$-dual to $\theta$. 
Usually, a compact convex symplectic manifold is called a convex symplectic domain. We have a natural contact form $\left.\theta\right|_{\partial M}$ on $\partial M$, and hence we call this the contact boundary of $M$.

Definition 2.2 Let $M$ be a manifold without boundary. We say that $(M, \theta)$ is a convex symplectic manifold if there exist constants $c_{1}<c_{2}<\cdots$ tending to infinity and an exhausting function $\phi: M \rightarrow \mathbb{R}$ such that $\left(\left\{\phi \leq c_{i}\right\}, \theta\right)$ is a compact convex symplectic manifold for each $i$. Exhausting here means proper and bounded from below. If the flow of $\lambda$ exists for all positive time, then $(M, \theta)$ is called complete. If there exists a constant $c>0$ such that for all $x \geq c,(\{\phi \leq c\}, \theta)$ is a compact convex symplectic manifold, then we say that $(M, \theta)$ is of finite type.

Definition 2.3 Let $\left(M, \theta_{t}\right)$ be a smooth family of convex symplectic manifolds with exhausting functions $\phi_{t}$. Suppose that for each $t \in[0,1]$, there are constants $c_{1}<c_{2}<$ ... tending to infinity and an $\epsilon>0$ such that for each $s$ in $(t-\epsilon, t+\epsilon)$ and $i \in \mathbb{N}$, $\left(\left\{\phi_{s} \leq c_{i}\right\}, \theta_{s}\right)$ is a compact convex symplectic manifold. Then $\left(M, \theta_{t}\right)$ is called a convex symplectic deformation.

The constants $c_{1}<c_{2}<\cdots$ mentioned in this definition depend on $t$ but not necessarily in a continuous way. The nice feature of convex symplectic manifolds is that we have some control over how they behave near infinity. That is, the level set $\phi^{-1}\left(c_{k}\right)$ is a contact manifold for all $k$. Also note that if we have a convex symplectic manifold of finite type then it has a cylindrical end. That is, there exists a manifold $N$ with a contact form $\alpha$ such that at infinity, $M$ is symplectomorphic to $(N \times[1, \infty), d(r \alpha))$ $(r$ is a coordinate on $[1, \infty)$ ).

Lemma 2.4 A compact convex symplectic manifold $M$ can be completed to a finite type complete convex symplectic manifold $(\widehat{M}, \theta)$.

This is explained for instance by Viterbo [29, Section 1.1]. The proof basically involves gluing a cylindrical end onto $\partial M$. Let $(M, \theta)$ be a complete convex symplectic manifold. Let $\left(M^{\prime}, \theta^{\prime}\right)$ be a compact convex symplectic manifold which is a codimension 0 exact submanifold of $(M, \theta)$ (ie $\left.\theta\right|_{M^{\prime}}=\theta^{\prime}+d R$ for some smooth function $R$ on $M^{\prime}$ ).

Lemma 2.5 We can extend the embedding $M^{\prime} \hookrightarrow M$ to an embedding $\widehat{M^{\prime}} \hookrightarrow M$.

Proof There exists a function $R: M^{\prime} \rightarrow \mathbb{R}$ such that $\theta^{\prime}=\theta+d R$. We can extend $R$ over the whole of $M$ such that $R=0$ outside some compact subset $K$ containing $M^{\prime}$. Let $\lambda^{\prime}$ be the $\omega$-dual of $\theta^{\prime}$. Let $F_{t}: M \rightarrow M$ be the flow of $\lambda^{\prime}$. This exists for 
all time because $M$ is complete and $\lambda^{\prime}=\lambda$ outside $K$. We have an embedding $\Phi:\left(\partial M^{\prime}\right) \times[1, \infty) \rightarrow M$ defined by $\Phi(a, t)=F_{\log t}(a)$. This attaches a cylindrical end to $M^{\prime}$ inside $M$, hence we have an exact embedding $\widehat{M^{\prime}} \rightarrow M$ extending the embedding of $M^{\prime}$.

Definition 2.6 A Stein manifold $(M, J, \phi)$ is a complex manifold $(M, J)$ with an exhausting plurisubharmonic function $\phi: M \rightarrow \mathbb{R}$ (ie $\phi$ is proper and bounded from below and $-d d^{c}(\phi)>0$ where $\left.d^{c}=J^{*} d\right)$. A Stein manifold is called subcritical if $\phi$ is a Morse function with critical points of index $<\frac{1}{2} \operatorname{dim}_{\mathbb{R}} M$. A manifold of the form $\phi^{-1}((-\infty, c])$ is called a Stein domain.

We can perturb $\phi$ so that it becomes a Morse function. From now on, if we are dealing with a Stein manifold, we will always assume that $\phi$ is a Morse function. The index of a critical point of $\phi$ is always less than or equal to $\frac{1}{2} \operatorname{dim}_{\mathbb{R}} M$. This is because the unstable manifolds of these critical points are isotropic submanifolds of $M$. Note that the definition of a Stein manifold in Eliashberg [13, Section 2] is that it is a closed holomorphic submanifold of $\mathbb{C}^{N}$ for some $N$. This has an exhausting plurisubharmonic function $|z|^{2}$. An important example of a subcritical Stein manifold is $\left(\mathbb{C}^{n}, i,|z|^{2}\right)$. The Stein manifold $(M, J, \phi)$ is a convex symplectic manifold $\left(M, \theta:=-d^{c} \phi\right)$. Note that $\lambda:=\nabla \phi$ where $\nabla$ is taken with respect to the metric $\omega(\cdot, J(\cdot))$. It is easy to see that $\nabla \phi$ is a Liouville vector field transverse to a regular level set of $\phi$ and pointing outwards. We call a Stein manifold complete or of finite type if the associated convex symplectic structure is complete or of finite type respectively.

Definition 2.7 If $\left(J_{t}, \phi_{t}\right)$ is a smooth family of Stein structures on $M$, then it is called a Stein deformation if the function $(t, x) \longrightarrow \phi_{t}(x)$ is proper and for each $t \in[0,1]$, there exists $c_{1}<c_{2}<\ldots$ tending to infinity and an $\epsilon>0$ such that for each $s$ in $(t-\epsilon, t+\epsilon)$ we have that $c_{k}$ is a regular value of $\phi_{s}$. This induces a corresponding convex symplectic deformation.

Example 2.8 An affine algebraic subvariety $M$ of $\mathbb{C}^{N}$ admits a Stein structure. This is because it has a natural embedding in $\mathbb{C}^{N}$, so we can restrict the plurisubharmonic function $\|z\|^{2}$ to this variety to make it into a Stein manifold. We can also use the following method to find a plurisubharmonic function on $M$. We first compactify $M$ by finding a projective variety $X$ with complex structure $i$ and an effective ample divisor $D$ such that $M=X \backslash D$ (for instance we can embed $M$ in $\mathbb{C}^{N} \subset \mathbb{P}^{N}$ and then let $X$ be the closure of $M$ in $\mathbb{P}^{N}$ ). There exists an ample line bundle $E \longrightarrow X$ associated to the divisor $D$. Choose a holomorphic section $s$ of $E$ such that 
$D=s^{-1}(0)$. Then ampleness means that we can choose a metric $\|\cdot\|$ such that its curvature form $\omega:=i F_{\nabla}$ is a positive $(1,1)$-form. Hence we have a Stein structure

$$
(M:=X \backslash D, J:=i, \phi:=-\log \|s\|) .
$$

Note that by [27, Lemma 8], this is of finite type.

For affine algebraic varieties we need a uniqueness theorem. This basically comes from the text following [25, Lemma 4.4]. The problem is that this text only deals with the case when our compactification divisor $D$ is a normal crossing divisor. But the methods used carry over to general divisors by using Hironaka's resolution theorem. In fact, the paper [25] uses Hironaka's resolution theorem in the same way as we will use it. We will repeat the argument here. Let $M$ be an algebraic variety.

Lemma 2.9 If $h_{1}$ and $h_{2}$ are Stein functions on $M$ constructed as in the previous example, then $(1-t) h_{1}+t h_{2}$ is a Stein deformation.

Proof If we can prove that all the critical points of $(1-t) h_{1}+t h_{2}$ stay inside a compact set for all $t \in[0,1]$, then this will prove that it is a Stein deformation. The way we do this is to look at this function in a neighbourhood of some compactification divisor of $M$. For $i=1,2$, let $X_{i}$ be projective varieties compactifying $M$. Let $E_{i}$ be ample line bundles on $X_{i}$ whose associated divisor is effective and ample and has support equal to $X_{i} \backslash M$. Suppose $h_{i}$ is equal to $-\log \left\|s_{i}\right\|_{i}$ where $s_{i}$ is a section of the line bundle $E_{i}$ such that $s_{i}^{-1}(0)$ has support equal to $X_{i} \backslash M$ and $\|\cdot\|_{i}$ is a metric on $E_{i}$.

By Hironaka's resolution theorem, we have that there exists a compactification $X$ by a normal crossing divisor $D$ such that it dominates $X_{1}$ and $X_{2}$ (i.e there is a surjective morphism $X \rightarrow X_{i}$ such that away from $D$ and $D_{i}$ it is an isomorphism). We pull back the line bundles, sections and metrics to $X$ and Stein functions via these morphisms. We now just apply [25, Lemma 4.4], and this gives us our result.

The following operation constructs a new Stein manifold from two old ones. This is used to construct our infinite family of Stein manifolds. We will let $(M, J, \phi),\left(M^{\prime}, J^{\prime}, \phi^{\prime}\right)$ be complete finite type Stein manifolds. Because these manifolds are complete and of finite type, they are the completions of compact convex symplectic manifolds $N, N^{\prime}$ respectively. In fact $N=\{\phi \leq R\}, N^{\prime}=\left\{\phi^{\prime} \leq R\right\}$ for some arbitrarily large $R$. Let $p$ (resp. $p^{\prime}$ ) be a point in $\partial N$ (resp. $\partial N^{\prime}$ ). The following theorem is proved in greater generality in [12] and [8, Theorem 9.4]. 
Theorem 2.10 There exists a connected finite type Stein manifold $\left(M^{\prime \prime}, J^{\prime \prime}, \phi^{\prime \prime}\right)$ such that $N^{\prime \prime}:=\left\{\phi^{\prime \prime} \leq R\right\}$ is biholomorphic to the disjoint union of $N$ and $N^{\prime}$ with $\left.\phi^{\prime \prime}\right|_{N}=\phi$ on $N$ and $\left.\phi^{\prime \prime}\right|_{N^{\prime}}=\phi^{\prime}$ on $N^{\prime}$. Also, the only critical point of $\phi^{\prime \prime}$ outside $N^{\prime \prime}$ has index 1 .

In this theorem, what we are doing is joining $N$ and $N^{\prime}$ with a 1 -handle and then extending the Stein structure over this handle, and then completing this manifold so that it becomes a Stein manifold. The Stein manifold $M^{\prime \prime}$ is called the end connect sum of $M$ and $M^{\prime}$, and we define $M \#_{e} M^{\prime}$ as this end connected sum. If $M$ and $M^{\prime}$ are Stein manifolds diffeomorphic to $\mathbb{C}^{n}$, then $M \#_{e} M^{\prime}$ is also diffeomorphic to $\mathbb{C}^{n}$.

Remark There is an example due to the author of a nonfinite type Stein manifold which is not equivalent to any finite type Stein manifold. This is described by Seidel in [25, Section 7], and is constructed as an infinite end-connect-sum.

\subsection{Lefschetz fibrations}

Throughout this section we will let $E$ be a compact manifold with corners whose boundary is the union of two faces $\partial_{h} E$ and $\partial_{v} E$ meeting in a codimension 2 corner. We will also assume that $\Omega$ is a 2 -form on $E$ and $\Theta$ a 1 -form satisfying $d \Theta=\Omega$. We let $S$ be a surface with boundary. Let $\pi: E \rightarrow S$ be a smooth map with only finitely many critical points (ie points where $d \pi$ is not surjective). Let $E^{\text {crit }} \subset E$ be the set of critical points of $\pi$ and $S^{\text {crit }} \subset S$ the set of critical values of $\pi$. For $s \in S$, let $E_{S}$ be the fibre $\pi^{-1}(s)$.

Definition 2.11 If for every $s \in S$ we have that $\Omega$ is a symplectic form on $E_{S} \backslash E^{\text {crit }}$ then we say that $\Omega$ is compatible with $\pi$.

Note that if $\Omega$ is compatible with $\pi$ then there is a natural connection (defined away from the critical points) for $\pi$ defined by the horizontal plane distribution which is $\Omega$-orthogonal to each fibre. If parallel transport along some path in the base is well defined then it is an exact symplectomorphism (an exact symplectomorphism is a diffeomorphism $\Phi$ between two symplectic manifolds $\left(M_{1}, d \theta_{1}\right)$ and $\left(M_{2}, d \theta_{2}\right)$ such that $\Phi^{*} \theta_{2}=\theta_{1}+d G$ where $G$ is a smooth function on $M_{1}$ ). From now on we will assume that $\Omega$ is compatible with $\pi$. We deal with Lefschetz fibrations as defined in [24]. We define $F$ to be some smooth fibre of $\pi$. 
Definition 2.12 $(E, \pi)$ is an exact Lefschetz fibration if:

(1) $\pi: E \longrightarrow S$ is a proper map with $\partial_{v} E=\pi^{-1}(\partial S)$ and such that $\left.\pi\right|_{\partial_{v} V}: \partial_{v} E \rightarrow$ $\partial S$ is a smooth fibre bundle. Also there is a neighbourhood $N$ of $\partial_{h} E$ such that $\left.\pi\right|_{N}: N \rightarrow S$ is a product fibration $S \times \operatorname{nhd}(\partial F)$ where $\left.\Omega\right|_{N}$ and $\left.\Theta\right|_{N}$ are pullbacks from the second factor of this product.

(2) There is an integrable complex structure $J_{0}$ (resp. $j_{0}$ ) defined on some neighbourhood of $E^{\text {crit }}$ (resp. $S^{\text {crit }}$ ) such that $\pi$ is $\left(J_{0}, j_{0}\right)$ holomorphic near $E^{\text {crit }}$. At any critical point, the Hessian $D^{2} \pi$ is nondegenerate as a complex quadratic form. We also assume that there is at most one critical point in each fibre.

(3) $\Omega$ is a Kähler form for $J_{0}$ near $E^{\text {crit }}$.

Sometimes we will need to define a Lefschetz fibration without boundary. This is defined in the same way as an exact Lefschetz fibration except that $E$, the fibre $F$ and the base $S$ are open manifolds without boundary. We replace "neighbourhood of $\partial_{h} E$ " in the above definition with an open set whose complement is relatively compact when restricted to each fibre. We also replace " $\partial_{v} E$ " with $\pi^{-1}(S \backslash K)$ where $K$ is a compact set in $S$. Also $\pi$ is obviously no longer a proper map, and we assume that the set of critical points is compact. From now on we will let $(E, \pi)$ be an exact Lefschetz fibration.

Lemma 2.13 [24, Lemma 1.5] If $\beta$ is a symplectic form on $S$ then $\omega:=\Omega+N \pi^{*} \beta$ is a symplectic form on $E$ for $N$ sufficiently large.

We really want our Lefschetz fibrations to be described as finite type convex symplectic manifolds.

Definition 2.14 A compact convex Lefschetz fibration is an exact Lefschetz fibration $(E, \pi)$ such that $\left(F,\left.\Theta\right|_{F}\right)$ is a compact convex symplectic manifold. A compact convex Lefschetz deformation is a smooth family of compact convex Lefschetz fibrations parameterized by $[0,1]$.

Note that by the triviality condition at infinity, all smooth fibres of $\pi$ are compact convex symplectic manifolds as long as the base $S$ is connected. From now on we will assume that $(E, \pi)$ is a compact convex Lefschetz fibration.

Theorem 2.15 Let the base $S$ be a compact convex symplectic manifold $\left(S, \theta_{S}\right)$. There exists a constant $K>0$ such that for all $k \geq K$ we have: $\omega:=\Omega+k \pi^{*}\left(\omega_{S}\right)$ is a symplectic form, and the $\omega-$ dual $\lambda$ of $\Theta+k \pi^{*} \theta_{S}$ is transverse to $\partial E$ and pointing outwards. 
(The proof is given in Section 4.) Note that this theorem also implies that if we have a compact convex Lefschetz deformation, then we have a corresponding compact convex symplectic deformation because we can smooth the codimension 2 corners slightly.

If we have a compact convex Lefschetz fibration, then we wish to extend the Lefschetz fibration structure over the completion $\widehat{E}$ of $E$. Here is how we naturally complete $(E, \pi)$ : The horizontal boundary is a product $\partial F \times S$. We can add a cylindrical end $G:=(\partial F \times[1, \infty)) \times S$ to this in the usual way (ie as in Lemma 2.4), extending $\Theta$ over this cylindrical end by the 1 -form $r\left(\left.\Theta\right|_{\partial F}\right)$ where $r$ is the coordinate for $[1, \infty)$. Let $E_{1}$ be the resulting manifold. We also extend the map $\pi$ over $E_{1}$ by letting $\left.\pi\right|_{G}: G \rightarrow S$ be the natural projection. This ensures that $\pi$ is compatible with the natural symplectic form on $E_{1}$ defined as in Lemma 2.13. The fibres of $\pi$ are finite type complete convex symplectic manifolds. We now need to "complete" the vertical boundary of $E_{1}$ so that we have a fibration over the completion $\hat{S}$ of $S$. Let $V:=\partial_{v} E_{1}:=\pi^{-1}(\partial S)$. We then attach $A:=V \times[0, \infty)$ to $E_{1}$ by identifying $V \subset E_{1}$ with $V \times\{0\} \subset A$ to create a new manifold $\widehat{E}$. We can extend our map $\pi$ over $A$ by letting $\left.\pi\right|_{A}(v, r)=\left.\pi\right|_{V}(v)$ where $v \in V \subset E_{1}$ and $r \in[0, \infty)$. Let $\pi_{1}: A \rightarrow V$ be the natural projection onto $V$. Let $\Theta_{A}:=\left.\pi_{1}^{*} \Theta\right|_{V}$. We wish to join $\Theta$ with $\Theta_{A}$ inside $\widehat{E}$. Let $\zeta:[0, \infty) \rightarrow[0,1]$ be a smooth function such that $\zeta(x)=0$ for $x<0.5$ and $\zeta(x)=1$ for $x>1$. The 1 -form $\Theta$ smoothly extends to a 1 -form $Q$ defined on $V \times[0, \epsilon] \subset A$ such that $d Q$ restricted to the fibres of $\pi$ is a symplectic form and $Q=\Theta$ in the region $G^{\prime}=(\partial F \times[1, \infty)) \times \widehat{S}$ (ie the region where the Lefschetz fibration is a product near infinity). Let $\Theta_{A}^{\prime}(v, r):=(1-\zeta(K r / \epsilon)) Q+\zeta(K r / \epsilon) \Theta_{A}$ where $K$ is a large constant. We can now smoothly extend $\Theta$ over $A$ by the 1 -form $\Theta_{A}^{\prime}$. Note that $d \Theta_{A}^{\prime}$ is a symplectic form on all the fibres for $K$ large enough because $\left.\Theta\right|_{V}=\left.\Theta_{A}\right|_{V \times\{0\}}$, and hence $d Q$ restricted to the fibres is as close as we like to $d \Theta_{A}$ restricted to the fibres. This means that $(1-t) d Q+t d \Theta_{A}$ is arbitrarily close to a symplectic form when restricted to the fibres and hence it is a symplectic form when restricted to the fibres for $t \in[0,1]$ and this implies that $d \Theta_{A}^{\prime}$ is a symplectic form on all the fibres.

Definition 2.16 $(\widehat{E}, \pi)$ is called the completion of $(E, \pi)$.

Note that the base of our completed fibration is $\left(\hat{S}, \theta_{S}\right)$.

Definition 2.17 Any fibration which is the completion of a compact convex Lefschetz fibration is called a complete convex Lefschetz fibration.

Note that if we add a large multiple of $\pi^{*} \theta_{S}$ to $\Theta$ then $(\widehat{E}, \theta)$ is a complete finite type convex symplectic manifold. Lefschetz fibrations have well defined parallel transport 
maps due to the fact that the fibration is trivial near the horizontal boundary of $E$. Now we need to deal with almost complex structures on $\widehat{E}$, as this will be useful when we later define $\mathrm{SH}_{*}^{\text {lef }}$. Let $J$ (resp. $j$ ) be an almost complex structure on $\widehat{E}$ (resp. $\widehat{S}$ ).

Definition 2.18 We say that $(J, j)$ are compatible with $(\widehat{E}, \pi)$ if:

(1) $\pi$ is $(J, j)$-holomorphic, and that $J=J_{0}$ near $E^{\text {crit }}$ and $j=j_{0}$ near $S^{\text {crit }}$.

(2) $j$ is convex at infinity with respect to the convex symplectic structure of $\hat{S}$ (ie $\theta_{S} \circ j=d r$ for large $r$ where $\theta_{S}$ is the contact form at infinity on $\widehat{S}$ and $r$ is the radial coordinate of the cylindrical end of $\widehat{S}$ ).

(3) $J$ is a product $\left(J_{F}, j\right)$ on the region $C \times S$ where $C$ is the cylindrical end $\partial F \times[1, \infty)$ of $\widehat{F}$, and $J_{F}$ is convex at infinity for $F .\left(\left.\theta\right|_{\widehat{F}} \circ J_{F}=d r\right.$ for large $r$ where $r$ is the radial coordinate of the cylindrical end).

(4) $\omega(\cdot, J \cdot)$ is symmetric and positive definite.

If $(\widehat{E}, \pi)$ is a complete convex Lefschetz fibration then the space of such almost complex structures is nonempty and contractible (see Seidel [24, Section 2.2]). We wish to have a slightly larger class of almost complex structures.

Definition 2.19 We define $\mathcal{J}^{h}(\widehat{E})$ to be the space of almost complex structures on $\widehat{E}$ such that for each $J$ in this space, there exists a $\left(J_{1}, j_{1}\right)$ compatible with $(\widehat{E}, \pi)$ and a compact set $K \subset \widehat{E}$ with $J=J_{1}$ outside $K$ and with $\omega(\cdot, J \cdot)$ symmetric and positive definite everywhere.

The set of such complex structures is still contractible.

\subsection{Symplectic homology}

In this section we will discuss symplectic homology as defined by Viterbo [29] for finite type Stein manifolds. For simplicity we will assume that our homology theory has coefficients in $\mathbb{Z} / 2$.

Let $(M, \theta)$ be a compact convex symplectic manifold. Our manifold $\widehat{M}$ has a cylindrical end symplectomorphic to $(N \times[1, \infty), d(r \alpha))$ where $r$ is a coordinate on $[1, \infty)$ and $\alpha$ is a contact form on $N$. We choose a smooth function $H: \mathbb{S}^{1} \times \widehat{M} \longrightarrow \mathbb{R}$ such that each $H_{t}$ is of the form $H_{t}=a r+b$ at infinity where $a$ and $b$ are some constants independent of $t$. We call such a Hamiltonian admissible. We also choose an $\mathbb{S}^{1}$ family of almost complex structures $J_{t}$ compatible with the symplectic form. We assume that $J_{t}$ is convex with respect to this cylindrical end outside some large compact set 
(ie $\theta \circ J_{t}=d r$ ). We call the constant $a$ the slope at infinity. We also say that $J_{t}$ is admissible.

We define an $\mathbb{S}^{1}$ family of vector fields $X_{H_{t}}$ by $\omega\left(X_{H_{t}}, \cdot\right)=d H_{t}(\cdot)$. The flow Flow $_{X_{H_{t}}}^{t}$ is called the Hamiltonian flow. A 1-periodic orbit of $H_{t}$ is a path of the form $l:[0,1] \rightarrow \widehat{M}, l(t):=$ Flow $_{X_{H_{t}}}^{t}(p)$ where $p \in \widehat{M}$ and $l(0)=l(1)$. We choose the cylindrical end and the slope of our Hamiltonians so that the union of the 1periodic orbits form a compact set. Let $F:=$ Flow $_{X_{H_{t}}}^{1}$, then we have a correspondence between 1-periodic orbits $x$ and fixed points $p$ of $F$. In particular we say that $x$ is nondegenerate if $\left.D F\right|_{p}: T_{p} \widehat{M} \rightarrow T_{p} \widehat{M}$ has no eigenvalue equal to 1 . We can also assume that the 1-periodic orbits of our Hamiltonian flow $X_{H_{t}}$ are nondegenerate. We call a Hamiltonian $H$ satisfying these conditions a nondegenerate admissible Hamiltonian or an admissible Hamiltonian with nondegenerate orbits.

From now on we will assume that $c_{1}(M)=c_{1}(\widehat{M})=0$. If we are given a trivialisation of the canonical bundle $\mathcal{K} \cong \mathcal{O}$, then for each orbit $x$, we can define an index of $x$ called the Robbin-Salamon index (This is equal to the Conley-Zehnder index taken with negative sign). The choice of these indices depend on the choice of trivialisation of $\mathcal{K}$ up to homotopy but the indices are canonical if $H_{1}(M)=0$. We denote this index by ind $(x)$. Let

$$
\mathrm{CF}_{k}(M, H, J):=\bigoplus_{\substack{\operatorname{Flow}_{X_{H_{t}}}^{1}(x)=x, \operatorname{ind}(x)=k}} \mathbb{Z} / 2\langle x\rangle
$$

For a 1 -periodic orbit $\gamma$ we define the action $A_{H}(\gamma)$ :

$$
A_{H}(\gamma):=-\int_{0}^{1} H(t, \gamma(t)) d t-\int_{\gamma} \theta
$$

This is the convention of [29] and [21]. This differs in sign from Seidel's convention in [25]. We will now describe the differential

$$
\text { ว: } \mathrm{CF}_{k}(M, H, J) \rightarrow \mathrm{CF}_{k-1}(M, H, J) .
$$

We consider curves $u: \mathbb{R} \times \mathbb{S}^{1} \longrightarrow \widehat{M}$ satisfying the perturbed Cauchy-Riemann equations

$$
\partial_{s} u+J_{t}(u(s, t)) \partial_{t} u=\nabla^{g_{t}} H
$$

where $\nabla^{g_{t}}$ is the gradient associated to the $\mathbb{S}^{1}$ family of metrics $g_{t}:=\omega\left(\cdot, J_{t}(\cdot)\right)$. For two periodic orbits $x_{-}, x_{+}$let $\bar{U}\left(x_{-}, x_{+}\right)$denote the set of all curves $u$ satisfying the Cauchy-Riemann equations such that $u(s, \cdot)$ converges to $x_{ \pm}$as $s \rightarrow \pm \infty$. This has a natural $\mathbb{R}$ action given by replacing the coordinate $s$ with $s+v$ for $v \in \mathbb{R}$. Let $U\left(x_{-}, x_{+}\right)$be equal to $\bar{U}\left(x_{-}, x_{+}\right) / \mathbb{R}$. For a $C^{\infty}$ generic admissible Hamiltonian and 
complex structure we have that $U\left(x_{-}, x_{+}\right)$is an ind $\left(x_{-}\right)-\operatorname{ind}\left(x_{+}\right)-1$ dimensional manifold (see Floer, Hofer and Salamon [14]). There is a maximum principle which ensures that all elements of $U\left(x_{-}, x_{+}\right)$stay inside a compact set $K$ (see Oancea [20, Lemma 1.5]). Hence we can use a compactness theorem (see for instance Bourgeois et al [4]) which ensures that if $\operatorname{ind}\left(x_{-}\right)-1=\operatorname{ind}\left(x_{+}\right)$, then $U\left(x_{-}, x_{+}\right)$is compact and hence a finite set. Let $\# U\left(x_{-}, x_{+}\right)$denote the number of elements of $U\left(x_{-}, x_{+}\right)$ mod 2. Then we have a differential:

$$
\begin{gathered}
\partial: \mathrm{CF}_{k}(M, H, J) \longrightarrow \mathrm{CF}_{k-1}(M, H, J) \\
\partial\left\langle x_{-}\right\rangle:=\sum_{\operatorname{ind}\left(x_{+}\right)=\operatorname{ind}\left(x_{-}\right)-1} \# U\left(x_{-}, x_{+}\right)\left\langle x_{+}\right\rangle
\end{gathered}
$$

By analysing the structure of 1-dimensional moduli spaces, one shows $\partial^{2}=0$ and defines $\mathrm{SH}_{*}(M, H, J)$ as the homology of the above chain complex. As a $\mathbb{Z} / 2 \mathbb{Z}$ module $\mathrm{CF}_{k}(M, H, J)$ is independent of $J$, but its boundary operator does depend on $J$. The homology group $\mathrm{SH}_{*}(M, H, J)$ depends on $M, H$ but is independent of $J$ up to canonical isomorphism. Note that for each $f \in \mathbb{R}$ we have a subcomplex generated by orbits of action $\leq f$. The reason why this is a subcomplex is because if $\bar{U}\left(x_{-}, x_{+}\right)$ is a nonempty set, then $A_{H}\left(x_{-}\right) \geq A_{H}\left(x_{+}\right)$. This means that the differential decreases action and hence if $x_{-}$is an orbit of action $\leq f$, then $\partial\left(x_{-}\right)$is a linear combination of orbits of action $\leq f$. The homology of such a complex is denoted by $\mathrm{SH}_{*}^{\leq f}(M, H, J)$.

If we have two nondegenerate admissible Hamiltonians $H_{1} \leq H_{2}$ and two admissible almost complex structures $J_{1}, J_{2}$, then there is a natural map:

$$
\mathrm{SH}_{*}\left(M, H_{1}, J_{1}\right) \longrightarrow \mathrm{SH}_{*}\left(M, H_{2}, J_{2}\right)
$$

This map is called a continuation map. This map is defined from a map $C$ on the chain level as follows:

$$
\begin{gathered}
C: \mathrm{CF}_{k}\left(M, H_{1}, J_{1}\right) \longrightarrow \mathrm{CF}_{k}\left(M, H_{2}, J_{2}\right) \\
\partial\left\langle x_{-}\right\rangle:=\sum_{\operatorname{ind}\left(x_{+}\right)=\operatorname{ind}\left(x_{-}\right)} \# P\left(x_{-}, x_{+}\right)\left\langle x_{+}\right\rangle
\end{gathered}
$$

where $P\left(x_{-}, x_{+}\right)$is the set of solutions of the following equations: Let $K_{s}$ be a smooth increasing family of admissible Hamiltonians joining $H_{1}$ and $H_{2}$ and $J_{s}$ a smooth family of admissible almost complex structures joining $J_{1}$ and $J_{2}$. Then for a $C^{\infty}$ generic family $\left(K_{s}, J_{s}\right)$, the set $P\left(x_{-}, x_{+}\right)$is the set of solutions to the parameterized Floer equations

$$
\partial_{s} u+J_{s, t}(u(s, t)) \partial_{t} u=\nabla^{g_{t}} K_{s, t}
$$


such that $u(s, \cdot)$ converges to $x_{ \pm}$as $s \rightarrow \pm \infty$. If we have another such increasing family admissible Hamiltonians joining $H_{1}$ and $H_{2}$ and another smooth family of admissible almost complex structures joining $J_{1}$ and $J_{2}$, then the continuation map induced by this second family is the same as the map induced by $\left(K_{s}, J_{S}\right)$. The composition of two continuation maps is a continuation map.

If we take the direct limit of all these maps with respect to admissible Hamiltonians ordered by $\leq$, then we get our symplectic homology groups $\mathrm{SH}_{*}(M)$. Supposing we have a family of Hamiltonians $\left(H_{\lambda}\right)_{\lambda \in \Lambda}$ ordered by $\leq$. We say that a family of Hamiltonians $\left(H_{i}\right)_{i \in I \subset \Lambda}$ is cofinal if for every $\lambda \in \Lambda$, there exists an $i \in I$ and a constant $a$ such that $H_{\lambda} \leq H_{i}+a$. The fact that continuation maps are natural ensures that we can also define $\mathrm{SH}_{*}(M)$ as the direct limit of all these maps with respect to any cofinal family of Hamiltonians. If we have a degenerate admissible Hamiltonian $H$, then we can still define symplectic Homology $\mathrm{SH}_{*}(H, J)$ as a direct limit $\mathrm{SH}_{*}\left(H_{k}, J_{k}\right)$ where $H_{k}$ and $J_{k}$ are $C^{\infty}$ generic making $\mathrm{SH}_{*}\left(H_{k}, J_{k}\right)$ well defined, and such that $H_{k}$ and $J_{k}$ tend to $H$ and $J$. The limit is taken with respect to continuation maps. This is independent of choices of $H_{k}$ and $J_{k}$ due to the naturality of continuation maps (see Viterbo [29, Remark 1.2]).

The symplectic homology groups also have a ring structure. The product is called the pair of pants product and it makes $\mathrm{SH}_{n+*}(M)$ into a $\mathbb{Z} / 2 \mathbb{Z}$-graded algebra where $n$ is the complex dimension of $M$. The product is defined in the following way: Take an admissible Hamiltonian $H_{1}$ and another admissible Hamiltonian $H_{2}$ such that $H_{1}=H_{2}$ outside some large compact set, and such that $H_{1}(1, \cdot)=H_{2}(0, \cdot)$ with all time derivatives. Then $H_{1} \# H_{2}$ is also admissible where $H_{1} \# H_{2}$ is defined as:

$$
H_{1} \# H_{2}(t, x):= \begin{cases}2 H_{1}(2 t, x) & \left(t \in\left[0, \frac{1}{2}\right]\right) \\ 2 H_{2}(2 t-1, x) & \left(t \in\left[\frac{1}{2}, 1\right]\right)\end{cases}
$$

We define a chain map (maybe after perturbing the Hamiltonians $H_{1}, H_{2}, H_{1} \# H_{2}$ slightly):

$$
\begin{aligned}
\mathrm{CF}_{k}\left(H_{1}, J\right) \otimes \mathrm{CF}_{j}\left(H_{2}, J\right) & \rightarrow \mathrm{CF}_{k+j-n}\left(H_{1} \# H_{2}, J\right), \\
x_{1} \otimes x_{2} & \rightarrow \sum_{\operatorname{ind}\left(x_{3}\right)=k+j-n} \# \mathcal{M}\left(H, J, x_{1}, x_{2}, x_{3}\right)\left\langle x_{3}\right\rangle .
\end{aligned}
$$

The set $\mathcal{M}\left(H, J, x_{1}, x_{2}, x_{3}\right)$ is the set of maps $u$ : $\mathbb{P}^{1} \backslash\{0,1, \infty\} \rightarrow M$ which satisfy some Floer type equations and such that each puncture converges to an orbit. The details of this are explained in [2], [1] and [25, Section 8]. This commutes with continuation maps and hence we can take the direct limit of these maps with respect to the ordering $\leq$ 
giving us a map

$$
\mathrm{SH}_{n+i}(M) \otimes \mathrm{SH}_{n+j}(M) \rightarrow \mathrm{SH}_{n+i+j}(M)
$$

This also has a unit in degree $n$ and is given by counting holomorphic planes [25, Section 8].

Suppose that $\left(M^{\prime}, \theta^{\prime}\right)$ is a compact convex symplectic manifold which is an exact submanifold of $M$, then there exists a natural map

$$
i: \mathrm{SH}_{*}(M) \longrightarrow \mathrm{SH}_{*}\left(M^{\prime}\right)
$$

called the transfer map. The composition of two of these transfer maps is another transfer map. These maps are introduced in [29, Section 2] and studied in [7, Section 3.3]. Let $N$ be a general convex symplectic manifold. Let $\left(W_{j}\right)_{j \in J}$ be the set of codimension 0 compact convex exact symplectic submanifolds. This is a directed system, where the morphisms are just inclusion maps. If $W_{j_{1}} \subset W_{j_{2}}$, then we have a transfer map $\mathrm{SH}_{*}\left(W_{j_{2}}\right) \rightarrow \mathrm{SH}_{*}\left(W_{j_{1}}\right)$. Because the transfer map points in the opposite direction (ie from $W_{j_{2}}$ to $W_{j_{1}}$ instead of $W_{j_{1}}$ to $W_{j_{2}}$ ), we have an inverse system $\left(\mathrm{SH}_{*}\left(W_{j}\right)\right)_{j \in J}$ where the morphisms now are transfer maps. Hence we can define the ring $\mathrm{SH}_{*}(N)$ as the inverse limit of this inverse system. Because the definition only involves codimension 0 exact symplectic manifolds, we have that it is invariant under exact symplectomorphism. We have that $\mathrm{SH}_{*}(N)$ is invariant under convex symplectic deformations (see McLean [19, Theorem 2.12]). We also have that for a compact convex symplectic manifold $M, \mathrm{SH}_{*}(M)=\mathrm{SH}_{*}(\widehat{M})$ [19, Theorem 2.12].

Symplectic homology $\mathrm{SH}_{*}(M)$ also has a natural $H_{1}(M)$ grading. Each orbit $x$ of some Hamiltonian $H$ is contained in some $H_{1}(M)$ class, and the definition of the differential ensures that $\partial x$ is a linear combination of orbits in the same $H_{1}(M)$ class. Also if we have two orbits $x_{1}$ and $x_{2}$ of some Hamiltonian $H_{1}$ and $H_{2}$ respectively, then they are in $H_{1}(M)$ classes $a$ and $b$. If we have a pair of pants, where two of the boundaries are in classes $a$ and $b$, then the third boundary is in the class $a+b$ (if we orient the boundaries correctly). This ensures that the pants product of $x_{1}$ and $x_{2}$ is a linear combination of orbits of $H_{1} \# H_{2}$ in the homology class $a+b$. Hence the pair of pants product for $\mathrm{SH}_{*}(M)$ is additive in $H_{1}(M)$.

Theorem 2.20 Let $M, M^{\prime}$ be finite type Stein manifolds of real dimension greater than 2, then $\mathrm{SH}_{*}\left(M \#_{e} M^{\prime}\right) \cong \mathrm{SH}_{*}(M) \times \mathrm{SH}_{*}\left(M^{\prime}\right)$ as rings. Also the transfer map $\mathrm{SH}_{*}\left(M \#_{e} M^{\prime}\right) \rightarrow \mathrm{SH}_{*}(M)$ is just the natural projection

$$
\mathrm{SH}_{*}(M) \times \mathrm{SH}_{*}\left(M^{\prime}\right) \rightarrow \mathrm{SH}_{*}(M) .
$$


Cieliebak [7] showed that the above theorem is true if we view $\mathrm{SH}_{*}$ as a vector space. We will prove that we have a ring isomorphism in Section 10.3.

\subsection{Symplectic homology and Lefschetz fibrations}

We need three theorems which relate symplectic homology to Lefschetz fibrations. These are the key ingredients in proving that our exotic Stein manifolds are pairwise distinct. The proofs of these theorems will be deferred to Section 5 and Section 6 . Theorem 2.22 is very close to Oancea's Künneth formula [21] but Theorems 2.24 and 2.25 are new and the main part of the story. Throughout this section we will let $\pi^{\prime}: E^{\prime} \rightarrow S^{\prime}$ be a compact convex Lefschetz fibration with fibre $F^{\prime}$. From now on we will assume that $c_{1}\left(E^{\prime}\right)=0$ and to make $\mathrm{SH}_{*}\left(E^{\prime}\right)$-graded we will choose a trivialisation of the canonical bundle of $E^{\prime}$. Note that when we talk about symplectic homology of a compact convex Lefschetz fibration, we mean the symplectic homology of its completion with respect to the convex symplectic structure. The fibration $\widehat{E^{\prime}}$ can be partitioned into three sets as follows:

(1) $E^{\prime} \subset \widehat{E^{\prime}}$.

(2) $A:=F_{e}^{\prime} \times \widehat{S}^{\prime}$, where $F_{e}^{\prime}:=\partial F^{\prime} \times \mathbb{R}_{\geq 1}$ is the cylindrical end of $\widehat{F}^{\prime}$.

(3) $B:=\widehat{E^{\prime}} \backslash\left(A \cup E^{\prime}\right)$.

The set $B$ is of the form $\left(A_{1} \times \mathbb{R}_{\geq 1}\right) \sqcup\left(A_{2} \times \mathbb{R}_{\geq 1}\right) \sqcup \cdots \sqcup\left(A_{n} \times \mathbb{R}_{\geq 1}\right)$, where $A_{i}$ is a mapping torus of the monodromy symplectomorphism around one of the boundary components of $S^{\prime}$. Figure 1 is a picture of the regions $E^{\prime}, A$ and $B$.

Let $\pi_{1}: A \rightarrow F_{e}^{\prime}$ be the natural projection onto $F_{e}^{\prime}$.

Definition 2.21 Let $H_{S^{\prime}}$ be an admissible Hamiltonian for the base $\widehat{S}^{\prime}$. Let $H_{F^{\prime}}$ be an admissible Hamiltonian for the fibre $\widehat{F}^{\prime}$. Assume that $H_{F^{\prime}}=0$ on $F^{\prime} \subset \widehat{F}^{\prime}$. The map $H: \widehat{E^{\prime}} \rightarrow \mathbb{R}$ is called a Lefschetz admissible Hamiltonian if $\left.H\right|_{A}=\pi^{*} H_{S^{\prime}}+\pi_{1}^{*} H_{F^{\prime}}$ and $\left.H\right|_{B}=\pi^{*} H_{S^{\prime}}$ outside some large compact set. We say that $H$ has slope $(a, b)$ if $H_{S^{\prime}}$ has slope $a$ at infinity and $H_{F}$ has slope $b$ at infinity.

Let $H$ be a Lefschetz admissible Hamiltonian and let $J$ be an admissible almost complex structure for $E^{\prime}$. We will call the pair $(H, J)$ a Lefschetz admissible pair. For generic $(H, J)$ we can define $\mathrm{SH}_{*}\left(E^{\prime}, H, J\right)$ (see Section 5 for more details). If $\left(H_{1}, J_{1}\right)$ is another generic Lefschetz admissible pair such that $H \leq H_{1}$, then there is a continuation map $\mathrm{SH}_{*}(H, J) \rightarrow \mathrm{SH}_{*}\left(H_{1}, J_{1}\right)$ induced by an increasing homotopy from $H$ to $H_{1}$ through Lefschetz admissible Hamiltonians. Hence, we have a direct limit $\mathrm{SH}_{*}^{l}\left(E^{\prime}\right):=\lim _{\rightarrow(H, J)} \mathrm{SH}_{*}(H, J)$ with respect to the ordering $\leq$ on Hamiltonians $H$. This has the natural structure of a ring with respect to the pair of pants product. 


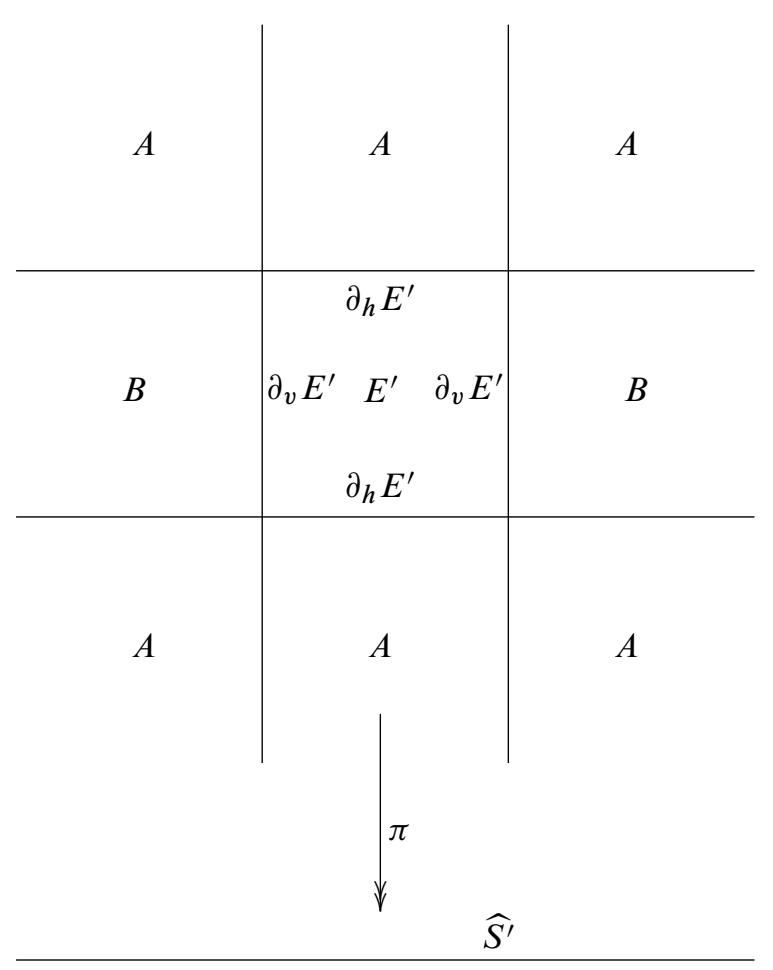

Figure 1

Theorem 2.22 There is a ring isomorphism $\mathrm{SH}_{*}\left(E^{\prime}\right) \cong \mathrm{SH}_{*}^{l}\left(E^{\prime}\right)$.

This will be proved in Section 5. Let $\epsilon$ be smaller than the length of the shortest Reeb orbit of $\partial F^{\prime}$. A Hamiltonian $H$ is called a half admissible Hamiltonian if it is Lefschetz admissible and has slope $(a, \epsilon)$. We let $J$ be an admissible almost complex structure for $E^{\prime}$.

Definition 2.23 We define

$$
\mathrm{SH}_{*}^{\text {lef }}\left(E^{\prime}\right):=\underset{(\overrightarrow{H, J})}{\lim _{\overrightarrow{1}}} \mathrm{SH}_{*}(H, J)
$$

as the direct limit with respect to the ordering $\leq$ on half admissible Hamiltonians $H$. This has the structure of a ring as usual.

The difference between $\mathrm{SH}_{*}\left(E^{\prime}\right)$ and $\mathrm{SH}_{*}^{l}\left(E^{\prime}\right)$ is that $\mathrm{SH}_{*}\left(E^{\prime}\right)$ is defined using Hamiltonians which are linear with respect to some fixed cylindrical end. The ring 
$\mathrm{SH}_{*}^{l}\left(E^{\prime}\right)$ is defined using Hamiltonians which are linear in the horizontal and vertical directions with respect to some Lefschetz fibration. The difference between $\operatorname{SH}_{*}^{\text {lef }}\left(E^{\prime}\right)$ and the other homology theories is that the slopes of a cofinal family of half admissible Hamiltonians do not have to tend to infinity pointwise in the vertical direction. This has to be true for $\mathrm{SH}_{*}\left(E^{\prime}\right)$ and $\mathrm{SH}_{*}^{l}\left(E^{\prime}\right)$ where the Hamiltonians have to get steeper and steeper at infinity in all directions. Because a half admissible Hamiltonian is Lefschetz admissible, we have a natural ring homomorphism

$$
\Phi: \operatorname{SH}_{*}^{\text {lef }}\left(E^{\prime}\right) \rightarrow \mathrm{SH}_{*}^{l}\left(E^{\prime}\right) .
$$

This comes from continuation maps $\mathrm{SH}_{*}(H, J) \rightarrow \mathrm{SH}_{*}(K, J)$ where $H$ is a half admissible Hamiltonian and $K$ is a Lefschetz admissible Hamiltonian. This is because $H \leq K$ when $K$ is large enough.

Theorem 2.24 If $S^{\prime}=\mathbb{D}$, the unit disk, then $\Phi$ is an isomorphism of rings. Hence by Theorem 2.22,

$$
\mathrm{SH}_{*}\left(E^{\prime}\right) \cong \mathrm{SH}_{*}^{\text {lef }}\left(E^{\prime}\right)
$$

as rings.

This will be proved in Section 5.1. Let $F^{\prime}$ (resp. $F^{\prime \prime}$ ) be a smooth fibre of $E^{\prime}$ (resp. $E^{\prime \prime}$ ). Let $F^{\prime}$ and $F^{\prime \prime}$ be Stein domains with $F^{\prime \prime}$ a holomorphic and symplectic submanifold of $F^{\prime}$.

Theorem 2.25 Suppose $E^{\prime}$ and $E^{\prime \prime}$ satisfy the following properties:

(1) $E^{\prime \prime}$ is a subfibration of $E^{\prime}$ over the same base.

(2) The support of all the monodromy maps of $E^{\prime}$ are contained in the interior of $E^{\prime \prime}$.

(3) Any holomorphic curve in $F^{\prime}$ with boundary inside $F^{\prime \prime}$ must be contained in $F^{\prime \prime}$.

Then $\mathrm{SH}_{*}^{\text {lef }}\left(E^{\prime}\right) \cong \mathrm{SH}_{*}^{\text {lef }}\left(E^{\prime \prime}\right)$ as rings.

This theorem will be proved in Section 6. Combining this theorem with Theorem 2.24 proves the key Theorem 1.3 in the introduction of this paper. 


\subsection{The Kaliman modification}

In order to produce examples of exotic symplectic manifolds, we first need to construct exotic algebraic varieties. One tool used for constructing these manifolds is called the Kaliman modification. Our treatment follows Section 4 of [30].

Consider a triple $(M, D, C)$ where $C \subseteq D \subseteq M$ are complex varieties. Let $M$ and $C$ be smooth, $D$ be an irreducible hypersurface in $M$, and $C$ be a closed subvariety contained in the smooth part of $D$ such that $\operatorname{dim}(C)<\operatorname{dim}(D)$.

Definition 2.26 (See Kaliman [18].) The Kaliman modification $M^{\prime}$ of $(M, D, C)$ is defined by $M^{\prime}:=\operatorname{Kalmod}(M, D, C)=\widetilde{M} \backslash \widetilde{D}$ where $\widetilde{M}$ is the blowup of $M$ along $C$ and $\widetilde{D}$ is the proper transform of $D$ in $\tilde{M}$.

The Kaliman modification of an affine variety is again an affine variety [18].

Lemma 2.27 [18, Theorem 3.5] Suppose that (i) $D$ is a topological manifold, and (ii) $D$ and $C$ are acyclic. Then $M^{\prime}$ is contractible if and only if $M$ is.

Example 2.28 (tom Dieck-Petrie surfaces [10; 11]) For $k>l \geq 2$ with $(k, l)$ coprime, the triple $A_{k, l}:=\left(\mathbb{C}^{2},\left\{x^{k}-y^{l}=0\right\},\{(1,1)\}\right)$ satisfies the conditions of Lemma 2.27. Hence $X_{k, l}=\operatorname{Kalmod}\left(A_{k, l}\right)$ is contractible. Note $X_{k, l}$ is isomorphic to

$$
\left\{\frac{(x z+1)^{k}-(y z+1)^{l}-z}{z}=0\right\} .
$$

Here $x, y, z$ are the standard coordinates of $\mathbb{C}^{3}$. Also the numerator of this fraction is divisible by $z$, hence the above fraction is a polynomial.

Here is another construction:

Example 2.29 (Kaliman [18]) If we have a contractible affine variety $M$ of complex dimension $n$, then we can construct a contractible affine variety

$$
M_{k}:=\operatorname{Kalmod}\left(M \times \mathbb{C}, M \times\left\{p_{1}, \ldots, p_{k}\right\},\left\{\left(a_{1}, p_{1}\right), \ldots,\left(a_{k}, p_{k}\right)\right\}\right)
$$

where $p_{i}$ are distinct points in $\mathbb{C}$ and $a_{i}$ are points in $M$. This variety is contractible by a repeated application of Lemma 2.27, because it is a repeated Kaliman modification with $D$ isomorphic to $M$ and $C$ a point. There are obvious variants: replace $\mathbb{C}$ and $\left\{p_{1}, \ldots, p_{k}\right\}$ with some contractible variety and a disjoint union of contractible irreducible hypersurfaces, etc. 
At the moment we are only discussing contractibility of varieties. We need to produce varieties diffeomorphic to some $\mathbb{C}^{n}$. We will use the h-cobordism theorem to achieve this stronger condition.

Corollary 2.30 (See Choudary and Dimca [6, page 174], Ramanujam [22] and Zaidenberg [30, Proposition 3.2].) Let $M$ be a contractible Stein manifold of finite type. If $n:=\operatorname{dim}_{\mathbb{C}} M \geq 3$ then $M$ is diffeomorphic to $\mathbb{C}^{n}$.

Proof Let $(J, \phi)$ be the Stein structure associated with $M$. We can also assume that $\phi$ is a Morse function. For $R$ large enough, the domain $M_{R}:=\{\phi<R\}$ is diffeomorphic to the whole of $M$ as $M$ is of finite type. We want to show that the boundary of $\bar{M}_{R}:=\{\phi \leq R\}$ is simply connected, then the result follows from the h-cobordism theorem.

The function $\psi:=R-\phi$ only has critical points of index $\geq n \geq 3$ because the function $\phi$ only has critical points of index $\leq n$ (see Eliashberg [13, Corollary 2.9]). Viewing $\psi$ as a Morse function, $\bar{M}_{R}$ is obtained from $\partial \bar{M}_{R}$ by attaching handles of index $\geq 3$. This does not change $\pi_{1}$, hence $\partial \bar{M}_{R}$ is simply connected because $\bar{M}_{R}$ is simply connected.

We now need a theorem which relates the Kaliman modification with symplectic homology. We do this via Lefschetz fibrations. Let $X, D, M$ be as in Example 2.8. Let $Z$ be an irreducible divisor in $X$ and $q \in(Z \cap M)$ a point in the smooth part of $Z$. We assume there is a rational function $m$ on $X$ which is holomorphic on $M$ such that $\overline{m^{-1}(0)}$ is reduced and irreducible and $Z=\overline{m^{-1}(0)}$. Let $M^{\prime}:=$ $\operatorname{Kalmod}(M,(Z \cap M),\{q\})$, and let $M^{\prime \prime}:=M \backslash Z$. Suppose also that $\operatorname{dim}_{\mathbb{C}} X \geq 3$. We also assume that $c_{1}\left(M^{\prime}\right)=c_{1}\left(M^{\prime \prime}\right)=0$.

Theorem 2.31 $\mathrm{SH}_{*}\left(M^{\prime \prime}\right)=\mathrm{SH}_{*}\left(M^{\prime}\right)$.

This theorem follows easily from the key Theorem 1.3 and the following theorem:

Theorem 2.32 There exist compact convex Lefschetz fibrations $E^{\prime \prime} \subset E^{\prime}$ respectively satisfying the conditions of Theorem 1.3 such that $E^{\prime}$ (resp. $E^{\prime \prime}$ ) is convex deformation equivalent to $M^{\prime}$ (resp. $M^{\prime \prime}$ ).

This will be proved in the appendix, Section 8 . The basic idea of the proof is to use Lefschetz fibrations defined in an algebraic way. 


\section{Proof of the main theorem}

\subsection{Construction of our exotic Stein manifolds}

First of all, we will construct a Stein manifold $K_{4}$ diffeomorphic to $\mathbb{C}^{4}$. We will then construct Stein manifolds $K_{n}$ diffeomorphic to $\mathbb{C}^{n}$ for all $n>3$ from $K_{4}$. Finally using end connect sums we will construct infinitely many Stein manifolds $\left(K_{n}^{k}\right)_{k \in \mathbb{N}}$ diffeomorphic to $\mathbb{C}^{n}$ for all $n>3$.

We define the polynomial $P\left(z_{0}, \ldots, z_{3}\right):=z_{0}^{7}+z_{1}^{2}+z_{2}^{2}+z_{3}^{2}$ and $V:=\{P=0\} \subset \mathbb{C}^{4}$. Let $\mathbb{S}^{7}$ be the unit sphere in $\mathbb{C}^{4}$.

\section{Theorem 3.1 [5] $\quad V \cap \mathbb{S}^{7}$ is homeomorphic to $\mathbb{S}^{5}$.}

Since $V$ is topologically the cone on the link $V \cap \mathbb{S}^{7}$, we have:

Corollary 3.2 $V$ is homeomorphic to $\mathbb{R}^{6}$.

Let $p \in V \backslash\{0\}$. We let $K_{4}:=\operatorname{Kalmod}\left(\mathbb{C}^{4}, V,\{p\}\right)$. Now by Corollary 3.2 and Lemma 2.27 we have that $K_{4}$ is contractible. Hence by Corollary 2.30 we have that $K_{4}$ is diffeomorphic to $\mathbb{C}^{4}$. We will now construct the varieties $K_{n}$ by induction. Suppose we have constructed the varieties $K_{4}, \ldots, K_{n}$, we wish to construct the variety $K_{n+1}$. We do this using Example 2.29. This means that we will define $K_{n+1}:=\operatorname{Kalmod}\left(K_{n} \times \mathbb{C}, K_{n} \times\{0\},(q, 0)\right)$ where $q$ is a point in $K_{n}$. All these are affine varieties and hence have Stein structures by Example 2.8. Finally, we define

$$
K_{n}^{k}:=\underset{\substack{\#_{e} \\ i=1 \ldots k}}{\#_{e}} K_{n}
$$

which is the $k$ fold end connect sum of $K_{n}$. The aim of this paper is to show that if $K_{n}^{k} \sim K_{n}^{m}$ then $k=m$.

\subsection{Proof of the main theorem in dimension 8}

Here we will prove Theorem 1.1 in dimension 8 . In this section we wish to show that if $K_{4}^{k} \sim K_{4}^{m}$ then $k=m$. Let $M^{\prime}:=K_{4}^{1}$. By Theorem 2.20, $\mathrm{SH}_{*}\left(K_{4}^{k}\right)=$ $\prod_{i=0}^{k} \mathrm{SH}_{*}\left(M^{\prime}\right)$. Hence if $i\left(M^{\prime}\right)$ is finite, $i\left(K_{4}^{k}\right)=i\left(M^{\prime}\right)^{k}$ where $i(M)$ denotes the number of idempotents of $\mathrm{SH}_{*}(M)$ for any Stein manifold $M$. So in order to distinguish these manifolds, we need to show that $1<i\left(M^{\prime}\right)<\infty$. Let $M^{\prime \prime}:=\mathbb{C}^{4} \backslash V$ where $V$ is defined in Section 3.1. By Theorem 2.31, we have that $\operatorname{SH}_{*}\left(M^{\prime \prime}\right)=$ $\mathrm{SH}_{*}\left(M^{\prime}\right)$. We have that $1<i\left(M^{\prime \prime}\right)<\infty$ by Theorem 7.7, hence $1<i\left(M^{\prime}\right)<\infty$. 


\subsection{Proof of the theorem in dimensions greater than 8}

Here we will prove Theorem 1.1 in dimensions greater than 8 . Let $K_{n}:=K_{n}^{1}$. For each $n>4$ we need to show that $1<i\left(K_{n}\right)<\infty$ in order to distinguish $K_{n}^{k}$. This is done by induction. Suppose that $1<i\left(K_{n}\right)<\infty$ for some $n$, then we wish to show that $1<i\left(K_{n+1}\right)<\infty$. We have by Theorem 2.31, that $\operatorname{SH}_{*}\left(K_{n+1}\right) \cong \operatorname{SH}_{*}\left(K_{n} \times \mathbb{C}^{*}\right)$. Let $B:=K_{n} \times \mathbb{C}^{*}$. Let $\mathrm{SH}_{*}^{\text {contr }}\left(\mathbb{C}^{*}\right)$ be the subring of $\mathrm{SH}_{*}\left(\mathbb{C}^{*}\right)$ with $H_{1}$ grading 0 .

One can check that $\mathrm{SH}_{*}^{\text {contr }}\left(\mathbb{C}^{*}\right)$ is a subring isomorphic to $H^{1-*}\left(\mathbb{C}^{*}\right)$. In particular $\mathrm{SH}_{1}^{\text {contr }}\left(\mathbb{C}^{*}\right) \cong \mathbb{Z} / 2$. By the Künneth formula [21], we have that

$$
\mathrm{SH}_{(n+1)+*}(B) \cong \mathrm{SH}_{n+*}\left(K_{n}\right) \otimes \mathrm{SH}_{1+*}\left(\mathbb{C}^{*}\right) .
$$

This ring is naturally graded by $H_{1}\left(\mathbb{C}^{*}\right)$. Hence any idempotent must be an element of

$$
\mathrm{SH}_{n+*}\left(K_{n}\right) \otimes \mathrm{SH}_{1+*}^{\mathrm{contr}}\left(\mathbb{C}^{*}\right) \subset \mathrm{SH}_{n+*}\left(K_{n}\right) \otimes \mathrm{SH}_{1+*}\left(\mathbb{C}^{*}\right)
$$

by Lemma 7.6. The ring $\mathrm{SH}_{1+*}^{\text {contr }}\left(\mathbb{C}^{*}\right)$ is naturally graded by the Conley-Zehnder index taken with negative sign because $c_{1}\left(\mathbb{C}^{*}\right)=0$. This means that any idempotents must live in

$$
\operatorname{SH}_{n+*}\left(K_{n}\right) \otimes \mathrm{SH}_{1}^{\text {contr }}\left(C^{*}\right) \cong \operatorname{SH}_{n+*}\left(K_{n}\right) \otimes \mathbb{Z} / 2 \cong \operatorname{SH}_{n+*}\left(K_{n}\right) .
$$

Hence $i\left(K_{n+1}\right)=i\left(K_{n}\right)$. This means that by induction we have $1<i\left(K_{n}\right)<\infty$ for all $n>3$ as we proved $1<i\left(K_{3}\right)<\infty$ in Section 3.2. This proves our theorem.

\section{Lefschetz fibration proofs}

Here we prove Theorem 2.15, which we restate:

Theorem 2.15 Let $(E, \pi)$ be a compact convex Lefschetz fibration. There exists a constant $K>0$ such that for all $k \geq K$ we have: $\omega:=\Omega+k \pi^{*}\left(\omega_{S}\right)$ is a symplectic form, and the $\omega-d u a l \lambda$ of $\theta:=\Theta+k \pi^{*} \theta_{S}$ is transverse to $\partial E$ and pointing outwards.

Proof We let $K$ be a large constant so that $\omega:=\Omega+\pi^{*}\left(K \omega_{S}\right)$ is a symplectic form (see Seidel [24, Lemma 1.5]). Let $\theta_{S}^{\prime}:=K \theta_{S}$ and $\omega_{S}^{\prime}=d \theta_{S}^{\prime}$ and $\lambda_{S}^{\prime}$ be the $\omega_{S}^{\prime}$-dual of $\theta_{S}$. Let $U \times V$ be some trivialisation of $\pi$ around some point $p \in \pi^{-1}(\partial S)$ where $U \subset F$ and $V \subset S$. We let $V$ be some small half disk around $\pi(p)$ and $U$ is some small open ball. Let $\pi^{1}: U \times V \rightarrow U$ be the natural projection. Let $\lambda_{F}$ be the $\left.\Omega\right|_{F}$-dual of $\left.\Theta\right|_{F}$, and $\lambda_{Q}$ be the horizontal lift of $\lambda_{S}^{\prime}$. The $\omega$-dual of $\Theta$ is equal to

$$
\lambda_{F}+W
$$


where $W$ is $\omega$-orthogonal to the vertical plane field tangent to the fibres and is equal to 0 near the horizontal boundary of $E$. The $\omega$-dual of $K \pi^{*} \theta_{S}^{\prime}$ is equal to

$$
G \lambda_{Q}
$$

where $G$ is some function on $U \times V$. This means that the $\omega$-dual of $\theta$ is

$$
\lambda=\lambda_{F}+W+G \lambda_{Q} .
$$

Because $W=0$ near the horizontal boundary and because the horizontal subspaces are tangent to the horizontal boundary, we have that $\lambda$ is transverse to the horizontal boundary. In order to show that $\lambda$ is transverse to the vertical boundary we need to ensure that we can make $G$ very large compared to $\lambda_{F}+W$. This can be done by making $K$ sufficiently large.

\section{A cofinal family compatible with a Lefschetz fibration}

In this section we construct a family of Hamiltonians $H_{k}: \widehat{E} \rightarrow \mathbb{R}$ which behave well with respect to the Lefschetz fibration, so that

$$
\mathrm{SH}_{*}^{l}(E):=\underset{k}{\lim } \mathrm{SH}_{*}\left(E, H_{k}, J\right)=\mathrm{SH}_{*}(E) .
$$

This would be obvious if $H_{k}$ belonged to the "usual" class (ie linear of slope $k$ on the cylindrical end) but it is not obvious that our Hamiltonians are linear with respect to some cylindrical end. Throughout this section, $(E, \pi)$ is a compact convex Lefschetz fibration. We let $\Theta, \Omega, \theta, \omega$ be defined as in Section 2.2.

Theorem 5.1 Let $H: \hat{E} \rightarrow \mathbb{R}$ be Lefschetz admissible for $E$ with nondegenerate orbits. Then the space of regular almost complex structures $\mathcal{J}_{\text {reg }}(\widehat{E}, H)$ is of second category in the space $\mathcal{J}^{h}(\widehat{E})$ of admissible almost complex structures with respect to the $C^{\infty}$ topology.

With a regular almost complex structure, it is possible to define symplectic homology $\mathrm{SH}_{*}(\widehat{E}, H, J)$ with the pair of pants product $\mathrm{SH}_{k}(\widehat{E}, H, J) \otimes \mathrm{SH}_{l}(\widehat{E}, H, J) \rightarrow$ $\mathrm{SH}_{l+k-n}(\widehat{E}, 2 H, J)$. Regular almost complex structures are almost complex structures such that some natural section of a Banach bundle associated with the almost complex structure is transverse to the zero section. This section is described by some linearized version of the perturbed Cauchy-Riemann equations. This theorem comes from using results of Floer, Hofer and Salamon [14]. Viterbo [29, Section 1.1] shows us why the Theorem is true in the context of open symplectic manifolds where the almost complex structure is fixed outside a large compact set and where there is a maximum 
principle as in [29, Lemma 1.8] or [20, Lemma 1.5]. This argument applies if we use the maximum principle in Lemma 5.2 below. This ensures that the moduli spaces of Floer trajectories are manifolds. For nongeneric $(H, J), \mathrm{SH}_{*}(H, J)$ is defined via small perturbations, and is independent of choice of small perturbation via continuation map techniques. We also need a maximum principle to ensure that the Floer moduli spaces have compactifications.

Let $W$ be a connected component of $\partial S$ where $S$ is the base. Now $\hat{S}$ has a cylindrical end $W \times[0, \infty)$. Let $r_{S}$ be the coordinate for $[1, \infty)$. Let $u: \mathbb{D} \rightarrow \widehat{E}$ satisfy Floer's equations for some $J \in \mathcal{J}^{h} \widehat{E}$ and some admissible Hamiltonian $H$. Here $\mathbb{D}$ is the unit disk parameterized by coordinates $(s, t)$. We can write $H=\pi^{*} H_{S}+\pi_{1}^{*} H_{F}$ as in Definition 2.21. We assume that $H_{F}=0$ on $F$.

Lemma 5.2 The function $f:=r_{S} \circ \pi \circ u$ cannot have an interior maximum for $r_{S}$ large.

Proof Let $f$ have an interior maximum at $q \in \mathbb{D}$. Let $U$ be a small neighbourhood of $u(q)$. The symplectic form $\omega$ on $\widehat{E}$ splits the tangent space of $E$ into vertical planes and horizontal planes. Let $V$ be the vertical plane field, and let $P$ be the horizontal plane field (the $\omega$-orthogonal of vertical tangent spaces of $\pi$ ). Let $\omega_{S}$ be the symplectic form on the base $S$, then $\omega_{P}:=\left.\pi^{*} \omega_{S}\right|_{P}$ is nondegenerate. This means that there exists a function

$$
g: \pi^{-1}(W \times[0, \infty)) \rightarrow(0, \infty)
$$

such that $g \omega_{P}=\left.\omega\right|_{P}$. We may assume that $J(P) \subset P$ because $J$ is compatible with $\widehat{E}$ if $r_{S}$ is large. Let $p$ be the natural projection $T E \rightarrow P$ induced by the splitting $T E=V \bigoplus P$.

Floer's equation for $u$ splits up into a horizontal part associated to $P$ and a vertical part associated to $V$. The horizontal part can be expressed as

$$
p\left(\frac{\partial u}{\partial s}\right)+J p\left(\frac{\partial u}{\partial t}\right)=-J \frac{1}{g} G
$$

where $G$ is a vector field on $P$ which is the $\omega_{P}$-orthogonal to $\left.d \pi^{*} H_{S}\right|_{P}$ in $P$. Hence $u^{\prime}:=\pi \circ u$ satisfies the equation

$$
\frac{\partial u^{\prime}}{\partial s}+j \frac{\partial u^{\prime}}{\partial t}=-j \frac{1}{u^{*}(g)} X_{H_{S}}
$$


where $j$ is the complex structure of $S$, and $X_{H_{S}}$ is the Hamiltonian vector field of $H_{S}$ in $S$. Rearranging the above equation gives

$$
u^{*}(g) \frac{\partial u^{\prime}}{\partial s}+j u^{*}(g) \frac{\partial u^{\prime}}{\partial t}=-j X_{H_{S}} .
$$

Now locally around the point $q$, we can choose a reparameterization of the coordinates $(s, t)$ to new coordinates $\left(s^{\prime}, t^{\prime}\right)$ so that $u^{\prime}$ satisfies

$$
\frac{\partial u^{\prime}}{\partial s^{\prime}}+j \frac{\partial u^{\prime}}{\partial t^{\prime}}=-j X_{H_{S}}
$$

(ie $\partial s^{\prime} / \partial s=\partial t^{\prime} / \partial t=1 / u^{*}(g)$ and $\left.\partial t^{\prime} / \partial s=\partial s^{\prime} / \partial t=0\right)$ ). The above equation is Floer's equation which doesn't have a maximum by [20, Lemma 1.5]. This gives us a contradiction as we assumed $f$ had a maximum at $q$.

If $H_{S}$ is a nondecreasing sequence of Lefschetz admissible Hamiltonians, then we can use the same methods as above to prove a maximum principle for $u$ satisfying the parameterized Floer equations

$$
\partial_{s} u+J_{s, t}(u(s, t)) \partial_{t} u=\nabla^{g_{t}} H_{s, t} .
$$

Note we also have a maximum principle in the vertical direction as well. We have that the region $A$ as defined in Definition 2.21 looks like $\partial F \times[1, \infty) \times \widehat{S}$. Let $r_{F}$ be the coordinate for $[0, \infty)$ in this product. Let $\pi_{1}: A \rightarrow \partial F \times[1, \infty)$ be the natural projection. If a Floer trajectory $u$ has an interior maximum with respect to $r_{F}$ for $r_{F}$ large, then $\pi_{1} \circ u$ satisfies Floer's equations on $F$ and hence has no maximum by [20, Lemma 1.5]. This gives us a contradiction. Hence $r_{F} \circ u$ has no maximum for $r_{F}$ large. The above maximum principles and the regularity result from Theorem 5.1 ensures that $\mathrm{SH}_{*}(\widehat{E}, H)$ is well defined.

Definition 5.3 Let $M$ be a manifold with contact form $\alpha$. Let $S:\{$ Reeb orbits $\} \rightarrow \mathbb{R}$, $S(o):=\int_{o} \alpha$. Then the period spectrum $\mathcal{S}(M)$ is the set $\operatorname{im}(S) \subset \mathbb{R}$. We say that the period spectrum is discrete and injective if the map $S$ is injective and the period spectrum is discrete in $\mathbb{R}$.

Definition 5.4 Let $H$ be a Hamiltonian on a symplectic manifold $M$. Then the action spectrum $\mathcal{S}(H)$ of $H$ is defined to be

$$
\mathcal{S}(H):=\left\{A_{H}(o): o \text { is a } 1 \text {-periodic orbit of } X_{H}\right\} .
$$

$A_{H}$ is the action defined in Section 2.3. 
We let $F$ be a smooth fibre of $(E, \pi)$ and $\Theta_{F}:=\left.\Theta\right|_{F}$. Also we let $S$ be the base of this fibration. Let $r_{S}$ and $r_{F}$ be the "cylindrical" coordinates on $\widehat{S}$ and $\widehat{F}$ respectively (ie $\omega_{S}=d\left(r_{S} \theta_{S}\right)$ on the cylindrical end at infinity and similarly with $r_{F}$ ). Let $W$ be some connected component of the boundary of $S$. Let $C:=\pi^{-1}(W) \times[1, \infty)$. Note: we will sometimes write $r_{S}$ instead of $\pi^{*} r_{S}$ so that calculations are not so cluttered. We hope that this will make things easier to understand for the reader.

The boundary of $E$ is a union of 2 manifolds whose boundaries meet at a codimension 2 corner. We can smooth out this corner so that $E$ becomes a compact convex symplectic manifold $M$ such that the completion $\widehat{M}$ is exact symplectomorphic to $\widehat{E}$. This means we can view $M$ as an exact submanifold of $\widehat{E}$. We will let $\partial M \times[1, \infty)$ be the cylindrical end of $\widehat{E}=\widehat{M}$ and we will let $r$ be the coordinate for the interval $[1, \infty)$. We will assume that the period spectrum of $\partial M$ is discrete and injective. Let $\varrho_{p}: \widehat{E} \rightarrow \mathbb{R}$ be an admissible Hamiltonian on $\widehat{M}=\widehat{E}$ with slope $p$ with respect to the cylindrical end $\partial M \times[1, \infty)$ where $p$ is a positive integer. We will also assume that $\varrho_{p}<0$ inside $M$ and that $\varrho_{p}$ tends to 0 in the $C^{2}$ norm inside $M$ as $p$ tends to infinity, and that $\varrho_{p}=h_{p}(r)$ in the cylindrical end. We assume that $h_{p}^{\prime}(r) \geq 0$ for all $r$ and $h_{p}^{\prime}(r)=p$ for $r \geq 2$. We also assume that $h_{p}^{\prime \prime}(r) \geq 0$ for all $r$. We can perturb the boundary of $M$ to ensure that no positive integer is in the period spectrum of $\partial M$ and hence $p$ is not in the action spectrum. Hence the family $\left(\varrho_{p}\right)_{p \in \mathbb{N}_{+}}$is a cofinal family of admissible Hamiltonians.

Theorem 5.5 There exists a cofinal family of Lefschetz admissible Hamiltonians $K_{p}: \widehat{E} \rightarrow \mathbb{R}$ and a family of almost complex structures $J_{p} \in \mathcal{J}_{\text {reg }}\left(\widehat{E}, K_{p}\right)$ such that for $p \gg 0$ :

(1) The periodic orbits of $K_{p}$ of positive action are in 1-1 correspondence with the periodic orbits of $\varrho_{p}$. This correspondence preserves index. Also the moduli spaces of Floer trajectories are canonically isomorphic between respective orbits.

(2) $K_{p}<0$ on $E \subset \widehat{E}$.

(3) $\left.K_{p}\right|_{E}$ tends to 0 in the $C^{2}$ norm on $E$ as $p$ tends to infinity.

This theorem implies that:

$$
\underset{p}{\lim } \mathrm{SH}_{*}^{[0, \infty)}\left(K_{p}\right)=\underset{p}{\lim } \mathrm{SH}_{*}\left(\varrho_{p}\right)
$$

$\mathrm{SH}_{*}^{[0, \infty)}\left(K_{p}\right):=\mathrm{SH}_{*}\left(K_{p}\right) / \mathrm{SH}_{*}^{(-\infty, 0)}\left(K_{p}\right)$ where $\mathrm{SH}_{*}^{(-\infty, 0)}$ is the symplectic homology group generated by orbits of negative action. We also have:

$$
\underset{p}{\lim } \mathrm{SH}_{*}\left(K_{p}\right)=\underset{p}{\lim } \mathrm{SH}_{*}^{[0, \infty)}\left(K_{p}\right)
$$


This is because there exists a cofinal family of Lefschetz admissible Hamiltonians $G_{p}$ such that:

(1) $G_{p}<0$ on $E \subset \widehat{E}$.

(2) $\left.G_{p}\right|_{E}$ tends to 0 in the $C^{2}$ norm on $E$ as $p$ tends to infinity.

(3) All the periodic orbits of $G_{p}$ have positive action.

Property (3) of $G_{p}$ will follow from Lemma 5.6. Using the fact that both $K_{p}$ and $G_{p}$ are cofinal, tending to 0 in the $C^{2}$ norm on $E$ and are nonpositive on $E$, there exist sequences $p_{i}$ and $q_{i}$ such that

$$
K_{p_{i}} \leq G_{q_{i}} \leq K_{p_{i+1}}
$$

for all $i$. Hence

$$
\underset{p}{\lim } \mathrm{SH}_{*}^{[0, \infty)}\left(G_{p}\right)=\underset{p}{\lim } \mathrm{SH}_{*}^{[0, \infty)}\left(K_{p}\right)
$$

Property (3) of $G_{p}$ implies

$$
\underset{p}{\lim } \mathrm{SH}_{*}^{[0, \infty)}\left(G_{p}\right)=\underset{p}{\lim } \mathrm{SH}_{*}\left(G_{p}\right)
$$

This gives us Equation (2). Combining this with Equation (1) gives

$$
\underset{p}{\lim } \mathrm{SH}_{*}\left(K_{p}\right)=\underset{p}{\lim } \mathrm{SH}_{*}\left(\varrho_{p}\right)
$$

This proves Theorem 2.22.

Before we prove Theorem 5.5, we need two preliminary Lemmas. We need a preliminary Lemma telling us something about the flow of a Lefschetz admissible Hamiltonian. We let $H=\pi^{*} H_{S}+\pi_{1}^{*} H_{F}$ be as in Definition 2.21. We assume that the slope of $H_{S}$ and $H_{F}$ is strictly less than some constant $B>0$. We set $H_{F}$ to be zero in $F$, and $H_{F}$ to be equal to $h_{F}\left(r_{F}\right)$ in the region $r_{F} \geq 1$ such that $h_{F}^{\prime}\left(r_{F}\right) \geq 0$ and $h_{F}^{\prime \prime}\left(r_{F}\right) \geq 0$. We also assume that for some very small $\epsilon>0, h_{F}^{\prime}$ is constant for $r_{F}>\epsilon$ and not in the period spectrum of $\partial F$ so that all the orbits lie in the region $r_{F} \leq \epsilon$. We define $H_{S}$ in exactly the same way so that it is zero in $S$ and equal to $h_{S}\left(r_{S}\right)$ on the cylindrical end of $\widehat{S}$ where $h_{S}$ has the same properties as $h_{F}$. The action of an orbit of $H_{F}$ in the cylinder $r_{F} \geq 1$ is $r_{F} h_{F}^{\prime}\left(r_{F}\right)-h_{F}\left(r_{F}\right)$ and similarly the action of an orbit of $H_{S}$ in $r_{S} \geq 1$ is $r_{S} h_{S}^{\prime}\left(r_{S}\right)-h_{S}\left(r_{S}\right)$, so we can choose $\epsilon$ small enough so that the actions of the orbits lie in the interval $[0, B]$ because the slope of $H_{S}$ and $H_{F}$ is less than $B$. We have from Section $4, \theta=\Theta+k \pi^{*} \theta_{S}$ where $\Theta$ is the 1 -form associated to the Lefschetz fibration (it is a 1 -form such that $\left.\Theta\right|_{F}$ makes each fibre $F$ into a compact convex symplectic manifold. Also $\theta_{S}$ is the 1 -form making the base $S$ into a compact convex symplectic manifold. The constant $k$ is some large constant. 
Lemma 5.6 For $k$ large enough, there exists a constant $\Xi$ depending only on $E$ and $\theta$ (not on $H$ ) such that the action of any orbit of $H$ is contained in the interval $[0, \Xi B]$.

Proof Inside $E$, we have that the Hamiltonian is 0 so all the orbits have action 0 there. In the region $A$ as defined in Definition 2.21, we have that the orbits come in pairs $(\gamma, \Gamma)$ where $\gamma$ is an orbit from $H_{S}$ and $\Gamma$ is an orbit of positive action from $H_{F}$. The action of $(\gamma, \Gamma)$ is the sum of the actions of $\gamma$ and $\Gamma$. Both these actions are positive. Also their actions are bounded above by $B$.

So we only need to consider orbits outside the region $A \cup E$. The Hamiltonian $\pi_{1}^{*} H_{F}$ is zero in this region so we only need to consider $\pi^{*} H_{S}$. We will consider the orbits of $\pi^{*} H_{S}$ in the region $r_{S} \geq 1$. In this region, there are no singular fibres of the Lefschetz fibration, so we have a well defined plane field $P$ which is the $\omega$-orthogonal plane field to the vertical plane field which is the plane field tangent to the fibres of $\pi$. The Hamiltonian flow only depends on $\left.\omega\right|_{P}$ and not the vertical plane field because $\pi^{*} H_{S}$ restricts to zero on the vertical plane field. The symplectic form $\left.\omega\right|_{P}$ is equal to $\left.G k \pi^{*} d \theta_{S}\right|_{P}$ for some function $G>0$. This means that the Hamiltonian vector field associated to $\pi^{*} H_{S}$ is $\frac{1}{G}$ times the horizontal lift of the Hamiltonian vector field associated to $H_{S}$ in $S$. Let $V$ be this horizontal lift. The construction of the completion of a Lefschetz fibration before Definition 2.16 ensures that the region $r_{S} \gg 1$ is a product $W \times[1, \infty)$ where $r_{S}$ parameterizes the second factor of this product and $\Theta$ is a pullback of a 1 -form on $W$ via the natural projection $W \times[1, \infty) \rightarrow W$. This means that $\Theta$ is invariant under translations in the $r_{S}$ direction (ie under the flow of the vector field $\partial / \partial r_{S}$ which is $1 / r_{S}$ times the horizontal lift of $\lambda_{S}$ where $\lambda_{S}$ is the Liouville flow in $\widehat{S}$ ). We also have that $d \theta_{S}$ is invariant under translations in the $r_{S}$ direction (ie under the flow of $\left.\left(1 / r_{S}\right) \lambda_{S}\right)$. Hence the symplectic structure $\omega$ is also invariant under translations in the $r_{S}$ direction for $r_{S} \gg 0$. This means that the function $G$ is bounded above and below by positive constants as the symplectic structure is invariant under translations in the $r_{S}$ direction and if we travel to infinity in the fibrewise direction (ie if we travel into the region $A$ ), then $G=1$. We want bounds on the function $V(\theta)$ because the function $G$ is bounded. Let $Y$ be the Hamiltonian flow of $r_{S}$ in $\widehat{S}$ and let $\tilde{Y}$ be its horizontal lift to $P$. We have that $Y\left(\theta_{S}\right)=1$. This means that $\widetilde{Y}\left(\pi^{*} \theta_{S}\right)=1$. We also have that $\tilde{Y}(\Theta)$ is bounded because $\Theta$ is invariant in the $r_{S}$ direction for $r_{S}$ large and $\tilde{Y}(\Theta)=0$ if we are near infinity in the fibrewise direction. We choose the constant $k$ large enough so that $\tilde{Y}(\theta)=\tilde{Y}(\Theta)+k \tilde{Y}\left(\pi^{*} \theta_{S}\right)>0$. This function is also bounded above because $\tilde{Y}(\Theta)$ is bounded and $k \tilde{Y}\left(\pi^{*} \theta_{S}\right)=k Y\left(\theta_{S}\right)=k$. This choice of $k$ only depends on the Lefschetz fibration and not on $H$. Now, $V=h_{S}^{\prime}\left(r_{S}\right) \tilde{Y}$. Because $h_{S}^{\prime}$ bounded below by 0 , we have that $V(\theta)$ is bounded below by 0 and bounded above by some constant multiplied by the slope of $H_{S}$. All the orbits of 
$H$ lie in some compact set where $H$ is $C^{0}$ small, so the action of an orbit is near $\int_{o} V(\theta) d x$ where the integral is taken over an orbit $o$ and $d x$ is the volume form on $o$ giving it a volume of 1 . This means that the action of these orbits is in the interval $[0, \Xi B]$ for some constant $\Xi$. This completes our theorem.

The manifold $\widehat{M}=\widehat{E}$ has a cylindrical end $\partial M \times[1, \infty)$. We let $r$ be the radial coordinate of this cylindrical end. The we define set $\{r \leq R\}$ to be equal to $M \cup(\partial M \times[1, R])$. We define the sets $\left\{r_{F} \leq R\right\}$ and $\left\{r_{S} \leq R\right\}$ in a similar way.

Lemma 5.7 There exists a constant $\varpi>0$ such that for all $R \geq 1$, we have that $\{r \leq R\} \subset\left\{r_{S} \leq \varpi R\right\}$ and $\{r \leq R\} \subset\left\{r_{F} \leq \varpi R\right\}$.

Proof We will deal with $r_{S}$ first. The level set $r=R$ is equal to the flow of $\partial M$ along the Liouville vector field $\lambda$ for a time $\log (R)$. Hence, all we need to do is show that $d r_{S}(\lambda)$ is bounded above by $e^{\varpi} r_{S}$. This means that if $p$ is a point in $\partial M$, then the rate at which $r_{S}(p)$ increases as we flow $p$ along $\lambda$ is bounded above by $e^{\varpi} r_{S}(p)$. Hence if we flow $p$ for a time $\log (R)$ to a point $q$, then $r_{S}(q) \leq \varpi R$ which is our result.

We will now show $d r_{S}(\lambda)$ is bounded above by $e^{\varpi} r_{S}$ to finish the first part of our proof. We let $\Theta$ be a 1-form associated to $E$ as constructed before Definition 2.16. Then $\theta=\Theta+\pi^{*} \theta_{S}$ where $\theta_{S}$ is a convex symplectic structure for the base $\hat{S}$. We have that $\omega=d \Theta+\pi^{*} d \theta_{S}$. The construction before Definition 2.16 ensures that the region $r_{S} \gg 1$ is a product $W \times[1, \infty)$ where $r_{S}$ parameterizes the second factor of this product and $\Theta$ is a pullback of a 1 -form on $W$ via the natural projection $W \times[1, \infty) \rightarrow W$. This means that $\Theta$ is invariant under translations in the $r_{S}$ direction. Hence $d \Theta$ is also invariant under these translations. Also $\pi^{*} d \theta_{S}$ is invariant under translations in the $r_{S}$ direction. All of this means that the vector field $V$ defined as the $\omega$-dual of $\Theta$ is invariant under these translations for $r_{S}$ large. This implies that $d r_{S}(V)$ is bounded.

Let $V^{\prime}$ be the $\omega$-dual of $\pi^{*} \theta_{S}$. Let $\lambda_{S}$ be the Liouville vector field in $\hat{S}$. Then $V^{\prime}=G L$ where $L$ is the horizontal lift of $\lambda_{S}$ and $G: \widehat{E} \rightarrow \mathbb{R}$ is defined in the proof of Lemma 5.6. The proof of Lemma 5.6, tells us that $G$ is a bounded function. Also, $d r_{S}\left(\lambda_{S}\right)=r_{S}$, hence

$$
d r_{S}\left(V^{\prime}\right)=G d r_{S}(L)=G d r_{S}\left(\lambda_{S}\right)=G r_{S}
$$

So $d r_{S}\left(V^{\prime}\right)$ is bounded above by some constant multiplied by $r_{S}$. Finally, we have that $\lambda=V+V^{\prime}$ which means that there exists a $\varpi>0$ such that $d r_{S}(\lambda)$ is bounded above by $e^{\varpi} r_{S}$. 
We will now deal with $r_{F}$. This is slightly more straightforward because the Lefschetz fibration is a product $\partial F \times[1, \infty) \times \widehat{S}$ and $\theta$ splits up in this product as $\Theta+\pi^{*} \theta_{S}$, where we can view $\Theta$ as 1 -form on $\partial F \times[1, \infty)$. We need to bound $d r_{F}(\lambda)$. In this case, because everything splits in this product, we have that $d r_{F}(\lambda)=d r_{F}(\Lambda)$ where $\Lambda$ is the $\omega$-dual of $\Theta$. This is equal to $r_{F} \leq e^{\varpi} r_{F}$ as $\varpi>0$. Hence we have that $r \leq R$ implies that $r_{F} \leq \varpi R$.

Proof of Theorem 5.5 Let $\varrho_{p}$ be the Hamiltonian as above. We will write $\varrho=\varrho_{p}$ for simplicity. The idea of the proof is to modify the Hamiltonian $\varrho$ outside some large compact set so that it becomes Lefschetz admissible and in the process only create orbits of negative action without changing the orbits of $\varrho$ or the Floer trajectories connecting orbits of $\varrho$. We will do this in three steps. In step (a), we will modify $\varrho$ to a Hamiltonian $\varsigma$ so that it becomes constant outside a large compact set $\kappa$ while only adding orbits of negative action. This is exactly the same as the construction due to Hermann [17]. In step (b) we will consider a Lefschetz admissible Hamiltonian $L$ which is 0 in the region $\kappa$, but has action bounded above so that the orbits of $L+\varsigma$ outside $\kappa$ have negative action. We define our cofinal family $K_{p}:=L+\varsigma$. In step (c) we ensure that the Floer trajectories and pairs of pants satisfying Floer's equation connecting orbits of positive action stay inside the region $r \leq 2$.

(a) We have that $p$ is the slope of the Hamiltonian $\varrho$ and this is not in the period spectrum of $\partial M$. Hence, we define $\mu:=\mu(p)>0$ to be smaller than the distance between $p$ and the action spectrum. Define

$$
A=A(p):=3 p / \mu>1 .
$$

We can assume that $A>4$ because we can choose $\mu$ to be arbitrarily small. Remember that $\widehat{E}=\widehat{M}$ where $M$ is a compact convex symplectic manifold, and that $r$ is the radial coordinate for the cylindrical end of $\widehat{M}$. We define $\varsigma$ to be equal to $\varrho$ on $r \leq A-1$. On the region $r \geq 1$, we have that $\varrho$ is equal to $h_{p}(r)$. We will just write $h$ instead of $h_{p}$. Set $\varsigma=k(r)$ for $r \geq 1$ with non negative derivative. This means that in the region $1 \leq r \leq A-1$ we have that $h(r)=k(r)$. Hence in $r \leq A-1$ we have that $k^{\prime \prime}(r) \geq 0$ and $k^{\prime}(r) \geq 0$, and in the region $2 \leq r \leq A-1$ we have $k^{\prime}(r)=p$. Also we have that $\varsigma$ is $C^{2}$ small and negative for $r$ near 1 . Because $\varsigma$ is $C^{2}$ small, we can also assume that $p$ is large enough so that for $r$ near $1, k^{\prime} \ll p$. Because $\varrho_{p}$ is cofinal, we can assume that $p$ is large enough so that $h(2)=k(2)>0$. Both these previous facts mean that $p(A-2)<k(A-1)<p(A-1)$. Outside this region, we define $k$ to be a function with the following constraints: For $r \geq A$ set $k(r)$ to be constant and equal to $C$ where $C=p(A-1)$. In the region $A-1 \leq r, k^{\prime \prime} \leq 0$. We assume that $k^{\prime} \geq 0$ for all $r \geq 1$. Figure 2 shows a picture. 


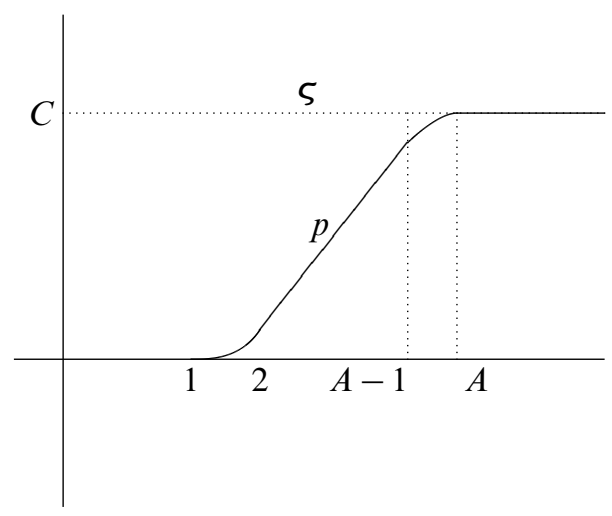

Figure 2

We want to show that the additional orbits of $\varsigma$ only have negative action. All these orbits lie in the region $r \geq 2$. In fact because $p$ is not in the action spectrum, they lie in the region $r \geq A-1$. In the region $\left\{r: p-\mu<k^{\prime}(r) \leq p\right\}$, we have that $\varsigma$ has no periodic orbits. Also, the action of a periodic orbit is $k^{\prime}(r) r-k(r)$. Combining these two facts implies that the action of a periodic orbit in the region $2 \leq r$ is less than

$$
(p-\mu) r-k(r) \leq(p-\mu) A-p(A-2)=-\mu A+2 p=-\mu \frac{3 p}{\mu}+2 p=-p<0 .
$$

Hence we have a Hamiltonian $\varsigma$ equal to $\varrho$ in the region $r \leq 2$ and such that it is constant and equal to $C=p(A-1)$ in the region $r \geq A-1$ and such that all the additional periodic orbits created have negative action.

(b) Lemma 5.6 tells us that there exists a cofinal family of Lefschetz admissible Hamiltonians $\Lambda_{p}$ such that the action spectrum of $\Lambda_{p}$ is bounded above by some constant $\Xi$ multiplied by the slope of $\lambda_{p}$. We can assume that both the slopes of $\lambda_{p}$ are equal to $\sqrt{(} p)$ (if $\sqrt{p}$ is in the action spectrum of the fibre or the base, then we perturb this value slightly to ensure that $\Lambda_{p}$ has orbits in a compact set). This means that the action of $\Lambda_{p}$ is bounded above by $\Xi \sqrt{p}$. The Hamiltonian $\Lambda_{p}$ is equal to zero in $E$. We will now define a Hamiltonian $L_{p}$ as follows: We let $\varpi$ be defined as in Lemma 5.7. Set $L_{p}=0$ in the region $\left\{r_{S} \leq \varpi A\right\} \cap\left\{r_{F} \leq \varpi A\right\}$. In the region $\left\{r_{S} \geq 1\right\} \cup\left\{r_{F} \geq 1\right\}$, we have that $\Lambda_{p}$ is a function of the form $\pi_{1}^{*} h_{F}\left(r_{F}\right)+\pi^{*} h_{S}\left(r_{S}\right)$. Here, $\pi_{1}$ is the natural projection $\partial F \times[1, \infty) \times \widehat{S} \rightarrow \partial F \times[1, \infty)$ (this is the same as the projection defined just before Definition 2.21). So, we set the function $\pi_{1}^{*} h_{F}\left(r_{F}\right)$ to be zero outside the domain of definition of $\pi_{1}$. Also, $\pi^{*} h_{S}$ is zero outside the 
region $r_{S} \geq 1$. We define $L_{p}$ to be

$$
\pi_{1}^{*} h_{F}\left(r_{F}-\varpi A\right)+\pi^{*} h_{S}\left(r_{S}-\varpi A\right)
$$

in the region $\left\{r_{S} \geq \varpi A\right\} \cup\left\{r_{F} \geq \varpi A\right\}$. Hence we have a well defined function $L_{p}$. Because $L_{p}$ has scaled up, we have that the action spectrum of $L_{p}$ is equal to $\varpi A$ multiplied by the action spectrum of $\Lambda_{p}$. Hence, we have that the action spectrum of $L_{p}$ is bounded above by $\varpi A \Xi \sqrt{p}$.

Because $\{r \leq A\} \subset\left\{r_{S} \leq A\right\} \cap\left\{r_{F} \leq A\right\}$, we can add $L_{p}$ to $\varsigma$ without changing the orbits of $\varsigma$ in the region $r \leq A$. Also, the action of the orbits of $\varsigma+L_{p}$ in the region $r \geq A$ is bounded above by $\varpi A \Xi \sqrt{p}-p(A-1)$. So for $p$ large enough we have that the additional orbits added are of negative action.

(c) We choose an almost complex structure $J \in \mathcal{J}^{h}(\widehat{E})$ such that on some neighbourhood of the hypersurface $r=2, J$ is admissible. Then [3, Lemma 7.2] and the comment after this Lemma ensure that no Floer trajectory or pair of pants satisfying Floer's equation connecting orbits inside $r<2$ can escape $r \leq 2$. Hence our Hamiltonian $K_{p}:=\varsigma+L_{p}$ has all the required properties.

\subsection{A better cofinal family for the Lefschetz fibration}

In this section we will prove Theorem 2.24. We consider a compact convex Lefschetz fibration $(E, \pi)$ fibred over the disc $\mathbb{D}$. Basically the cofinal family is such that $H_{F}=0$. This means that the boundary of $F$ does not contribute to symplectic homology of the Lefschetz fibration. The key idea is that near the boundary of $F$ the Lefschetz fibration looks like a product $\mathbb{D} \times \operatorname{nhd}(\partial F)$ and because symplectic homology of the disc is 0 we should get that the boundary contributes nothing. Recall the statement of Theorem 2.24:

$$
\mathrm{SH}_{*}(E) \cong \mathrm{SH}_{*}^{\text {lef }}(E) \text {. }
$$

We will define $F, S(=\mathbb{D}), r_{S}, r_{F}, \pi_{1}$ as in the previous section. This means that the compact convex symplectic manifold $F$ is a fibre of $E$ and $S$ is the base which in this section is equal to $\mathbb{D}$. We also have that $r_{S}$ is a radial coordinate for the cylindrical end of $\widehat{S}$ which we also identify with $\pi^{*} r_{S}$. The map $\pi_{1}$ is the natural projection $(\partial F \times[1, \infty)) \times \widehat{S} \rightarrow(\partial F \times[1, \infty))$ where $(\partial F \times[1, \infty)) \times \widehat{F}$ is a subset of $\widehat{E}$. The function $r_{F}$ is a radial coordinate for the cylindrical end of $\hat{F}$ which we also identify with $\pi_{1}^{*} r_{F}$. Before we prove Theorem 2.24 , we will write a short lemma on the $\mathbb{Z}$ grading of $\mathrm{SH}_{*}(E)$. 
Lemma 5.8 Let $\widehat{F}:=\pi^{-1}(a) \subset \widehat{E}(a \in \mathbb{D})$. Suppose we have trivialisations of $\mathcal{K}_{\hat{E}}$ and $\mathcal{K}_{\widehat{S}}$ (these are the canonical bundles for $\widehat{E}$ and $\widehat{S}$ respectively); these naturally induce a trivialisation of $\mathcal{K}_{\widehat{F}}$ away from $F$. If we smoothly move $a$, then this smoothly changes the trivialisation.

Proof We choose a $J \in \mathcal{J}^{h}(E)$. The bundle $E$ away from $E^{\text {crit }}$ has a connection induced by the symplectic structure. Let $A \subset \widehat{E}$ be defined as in Definition 2.21. Let $U$ be a subset of $A$ where:

(1) $\pi$ is $J$ holomorphic.

(2) $U$ is of the form $r \geq K$ where $r$ is the coordinate for $[1, \infty)$ in $A$ (see Definition 2.21).

This means that in $U$, we have that the horizontal plane bundle $\mathbb{H}$ is $J$ holomorphic. Choose a global holomorphic section of $\mathcal{K}_{\hat{S}}$ and lift this to a section $s$ of $\mathbb{H}$. Choose a global holomorphic section $t$ of $\mathcal{K}_{\hat{E}}$. The tangent bundle of $\widehat{F}$ is isomorphic to the $\omega$-orthogonal bundle $T$ of $\mathbb{H}$. This is also a holomorphic bundle. Let $\Lambda^{k} T$ be the highest exterior power of $T$. There exists a unique holomorphic section $w$ of $\Lambda^{k} T$ such that $s \wedge w=t$. Hence, $w$ is our nontrivial holomorphic section of $T$ in $U \cup \widehat{F}$. This can be extended to $A \cup \widehat{F}$ by property (2).

In the following proof, whenever we talk about indices of orbits of $\widehat{F}$ outside $F$, we do this with respect to the trivialisation of Lemma 5.8 above. We do not deal with orbits inside $F$ so this trivialisation is sufficient.

Proof of Theorem 2.24 We start by defining a cofinal family of Lefschetz admissible Hamiltonians $H^{\lambda}=\pi^{*} H_{S}^{\lambda}+\pi_{1}^{*} H_{F}^{\lambda}$. To avoid cluttered notation, we suppress the $\lambda$ and just write $H, H_{S}, H_{F}$ instead of $H^{\lambda}, H_{S}^{\lambda}, H_{F}^{\lambda}$ unless we need to explicitly deal with $\lambda$. We assume that the period spectrum of $\partial F$ is discrete and injective and also that the Reeb orbits of $\partial F$ are nondegenerate. We assume that $H_{F}=0$ on $F$ and is equal to $h_{F}\left(r_{F}\right)$ outside $F$ with $h_{F}^{\prime}\left(r_{F}\right)>0$ and $h_{F}^{\prime \prime}\left(r_{F}\right) \geq 0$ when $r_{F}>1$. The orbits of $H_{F}$ consist of constant orbits in $F$ and $\mathbb{S}^{1}$ families of orbits corresponding to periodic Reeb orbits outside $F$. We can perturb $H_{F}$ by a very small amount outside $F$ so that each $\mathbb{S}^{1}$ family of Reeb orbits becomes a pair of nondegenerate orbits (see Oancea [20, Section 3.3]). Hence, we have a Hamiltonian $H_{F}$ which is equal to 0 inside $F$ and all its orbits outside $F$ are nondegenerate. We set the slope of $H_{F}$ at infinity to be equal to $\lambda \notin \mathcal{S}(\partial F)$. The completion $\widehat{\mathbb{D}}$ of the disc $\mathbb{D}$ is symplectomorphic to $\mathbb{C}$ with the standard symplectic structure. We have that $r_{S}(z)=|z|^{2}$ where $z \in \mathbb{C}$. The function $r_{S}$ is defined only on the cylindrical end, but we will extend it to the 
interior of $\mathbb{D}$ by the function $|z|^{2}$. We set $H_{S}=\kappa(\lambda) r_{S}$ on $\mathbb{C}$ where $\kappa(\lambda)$ is some function of the slope $\lambda$ of $H_{F}$ such that it is never a multiple of $\pi$. Here $H_{S}$ has exactly one periodic orbit at $0 \in \mathbb{C}$ of index $(2 a+1)$ where $a$ is the integer satisfying $a \pi<\kappa(\lambda)<\pi(a+1)$ (see Oancea [20, Section 3.2]). For each $\lambda$, we choose $\kappa(\lambda)$ to be large enough so that the index of the only orbit of $H_{S}$ is greater than $M+\lambda+1$ where $M=-\operatorname{ind}(\Gamma)$ such that $\Gamma$ is an orbit of $H_{F}$ of lowest index. We also assume that as $\lambda$ tends to infinity, $\kappa(\lambda)$ also tends to infinity. The Hamiltonians $H^{\lambda}$ form a cofinal family of Lefschetz admissible Hamiltonians. Hence for some $J \in \mathcal{J}^{h}(\widehat{E})$, we have $\mathrm{SH}_{*}(E)=\lim _{\longrightarrow} \mathrm{SH}_{*}\left(E, H^{\lambda}, J\right)$.

We will also define a half Lefschetz admissible Hamiltonian $\bar{H}^{\lambda}:=\pi^{*} H_{S}^{\lambda}+\pi_{1}^{*} \bar{H}_{F}^{\lambda}$. When appropriate, we write $\bar{H}, \bar{H}_{F}$ instead of $\bar{H}^{\lambda}, \bar{H}_{F}^{\lambda}$. We define $\bar{H}_{F}=0$ inside $F$ and $\bar{H}_{F}:=\bar{h}_{F}\left(r_{F}\right)$ in the region $\left\{r_{F} \geq 1\right\}$ where the derivative $\bar{h}_{F}^{\prime}$ is so small that $\bar{H}_{F}$ has no periodic orbits in the region $\left\{r_{F}>1\right\}$. The Hamiltonians $\bar{H}^{\lambda}$ form a cofinal family of half admissible Hamiltonians, hence $\operatorname{SH}_{*}^{\text {lef }}(E)=\lim _{\longrightarrow} \mathrm{SH}_{*}\left(E, \bar{H}^{\lambda}, J\right)$.

We will construct a natural continuation map $\mathrm{SH}_{*}\left(\bar{H}^{\lambda}, J\right) \rightarrow \mathrm{SH}_{*}\left(H^{\lambda}, J\right)$ such that it is an isomorphism in all degrees less than $\lambda$. This will prove the theorem for the following reason: Because continuation maps are natural, we can take direct limits with respect to $\lambda$, so that we get a map $\mathrm{SH}_{*}(E) \rightarrow \mathrm{SH}_{*}^{\text {lef }}(E)$. This map must be an isomorphism because if we choose an integer $b$, then for $\lambda>b$ we have $\mathrm{SH}_{b}\left(\bar{H}^{\lambda}, J\right) \rightarrow \mathrm{SH}_{b}\left(H^{\lambda}, J\right)$ is an isomorphism which implies that $\mathrm{SH}_{b}(E) \rightarrow \mathrm{SH}_{b}^{\text {lef }}(E)$ is an isomorphism (because we are taking a direct limit as $\lambda$ tends to infinity). Hence $\mathrm{SH}_{*}(E) \rightarrow \mathrm{SH}_{*}^{\text {lef }}(E)$ is an isomorphism.

We will now show that the continuation map $\mathrm{SH}_{*}\left(\bar{H}^{\lambda}, J\right) \rightarrow \mathrm{SH}_{*}\left(H^{\lambda}, J\right)$ is an isomorphism in all degrees less than $\lambda$. From now on, we will write $H, H_{S}, H_{F}, \bar{H}, \bar{H}_{F}$ instead of $H^{\lambda}, H_{S}^{\lambda}, H_{F}^{\lambda}, \bar{H}^{\lambda}, \bar{H}_{F}^{\lambda}$. The region $\left\{r_{F} \geq 1\right\}$ is a product $([1, \infty) \times \partial F) \times \mathbb{C}$ and the orbits of the Hamiltonian $H$ come in pairs $(\gamma, \Gamma)$, where $\gamma$ is the orbit of $H_{S}$ and $\Gamma$ is a nonconstant orbit of $H_{F}$. The index of this orbit is the sum $\operatorname{ind}(\gamma)+\operatorname{ind}(\Gamma)$, hence its index is greater than $\lambda+1$. This means that all the orbits of index $\leq \lambda+1$ are disjoint from the region $\left\{r_{F} \geq 1\right\}$. We have that $H_{F}$ is not quite a function of $r_{F}$ in the region $\left\{r_{F} \geq 1\right\}$, as we perturbed it so that it had nondegenerate orbits. Having said that we can assume that for some $\delta>0$, we have $H_{F}=h_{F}\left(r_{F}\right)$ in the region $\left\{1 \leq r_{F} \leq 1+\delta\right\}$. We also assume that the Lefschetz admissible almost complex structure $J$ is of the form $j+J_{F}$ in the region $\left\{r_{F} \geq 1\right\}$ viewed as a subset of $\widehat{E}$, where $j$ is an complex structure on $\mathbb{C}$ and $J_{F}$ is convex on the cylindrical end of $\widehat{F}$. Any Floer trajectory satisfying Floer's equation with respect to $(H, J)$ connecting orbits outside the region $\left\{r_{F}>1\right\}$, must stay outside this region for the following reason: If $u: S \rightarrow \widehat{E}$ is such a curve, then let $\bar{S}:=u^{-1}\left(\left\{r_{F}>1\right\}\right)$. We can project 
$\left.u\right|_{\bar{S}}: \bar{S} \rightarrow\left\{r_{F}>1\right\}$ down from $\left\{r_{F}>1\right\}$ to $(1, \infty) \times \partial F$. Lemma 7.2 from [3] then tells us that this curve cannot exist. Hence $\bar{S}$ is empty and the claim follows. A similar argument shows that if we had a pair of pants satisfying Floer's equation with respect to Hamiltonians similar to $H$ connecting orbits outside $\left\{r_{F} \geq 1\right\}$, then the curve must also be disjoint from $\left\{r_{F} \geq 1\right\}$. Similarly any Floer trajectory or pair of pants satisfying Floer type equations with respect to $(\bar{H}, J)$ must be disjoint from $\left\{r_{F} \geq 1\right\}$ because all orbits of $\bar{H}$ are disjoint from this region. Let $K_{s}: \widehat{F} \rightarrow \mathbb{R}$ be a monotone increasing sequence of admissible Hamiltonians joining $\bar{H}_{F}$ and $H_{F}$ such that in the region $\left\{1 \leq r_{F} \leq 1+\delta\right\}$, we have that $K_{S}$ is a function of $r_{F}$ only. Again if we have a trajectory satisfying the Floer continuation equations with respect to $\left(K_{S}, J\right)$ joining orbits of $\bar{H}$ and $H$ which are disjoint from $\left\{r_{F} \geq 1\right\}$, then by [3, Lemma 7.2] we have that this trajectory is also disjoint from $\left\{r_{F} \geq 1\right\}$. Combining all these facts, we get that the continuation map induced by $\left(K_{S}, J\right)$ is an isomorphism in all degrees less than $\lambda$ because $\bar{H}=H$ outside the region $\left\{r_{F} \geq 1\right\}$ and all orbits of $H$ and $\bar{H}$ of degree less than $\lambda+1$ along with all Floer trajectories and pairs of pants connecting them are disjoint from this region. This completes the proof of Theorem 2.24.

\section{$6 \mathrm{SH}_{*}^{\text {lef }}(\widehat{E})$ and the Kaliman modification}

In this section we prove Theorem 2.25. Throughout this section we assume that $E^{\prime}$ and $E^{\prime \prime}$ are Lefschetz fibrations as described in Section 2.4. We recall the situation:

(1) $E^{\prime \prime}$ is a subfibration of $E^{\prime}$ over the same base.

(2) The support of the parallel transport maps of $E^{\prime}$ are contained in the interior of $E^{\prime \prime}$.

(3) There exists a complex structure $J_{F^{\prime}}$ (coming from a Stein domain) on $F^{\prime}$ such that any $J_{F^{\prime}}$-holomorphic curve in $F^{\prime}$ with boundary in $F^{\prime \prime}$ must be contained in $F^{\prime \prime}$.

We wish to prove that $\mathrm{SH}_{*}^{\text {lef }}\left(E^{\prime}\right) \cong \mathrm{SH}_{*}^{\text {lef }}\left(E^{\prime \prime}\right)$ as rings.

Proof of Theorem 2.25 Fix $\lambda>0$. The value $\lambda$ is going to be the slope of some Hamiltonian, we can always perturb $\lambda$ slightly so that it isn't in the action spectrum of the boundary. By Theorem 9.1 we can choose an almost complex structure $J_{F^{\prime}, 1}$ on $\widehat{F}^{\prime}$ after a convex deformation away from $F^{\prime}$ such that it is convex with respect to some cylindrical end at infinity and such that any $J_{F^{\prime}, 1}$-holomorphic curve in $F^{\prime}$ with boundary in $F^{\prime \prime}$ must be contained in $F^{\prime \prime}$. The reason is because we can ensure that $J_{F^{\prime}, 1}=J_{F^{\prime}}$ in $F^{\prime} \subset \widehat{F}^{\prime}$ and that any $J_{F^{\prime}, 1}$-holomorphic curve with boundary in 
$F^{\prime \prime} \subset F^{\prime}$ is contained in $F^{\prime}$ by Theorem 9.1 hence is contained in $F^{\prime \prime}$ by property (3) above. Supposing we have a Hamiltonian $H_{F^{\prime}}$ which is of the form $h_{F^{\prime}}\left(r_{F^{\prime}}\right)$ on the cylindrical end where $r_{F^{\prime}}$ is the radial coordinate and $h^{\prime} \geq 0$ and $H_{F^{\prime}}=0$ elsewhere. Then, any curve (Floer cylinder or pair of pants) with boundary in $F^{\prime \prime}$ satisfying Floer's equations with respect to $H_{F^{\prime}}$ and $J_{F^{\prime}, 1}$ must be contained in $F^{\prime \prime}$. We choose $h^{\prime}$ small enough so that $H_{F^{\prime}}$ has no periodic orbits in the region $r_{F^{\prime}}>1$. The convex deformation mentioned in Theorem 9.1 fixes $F^{\prime} \subset \widehat{F}^{\prime}$ hence it induces a convex deformation on $\widehat{E}$. This is because the region where we deform $\widehat{E}$ looks like a product $\mathbb{C} \times\left(\widehat{F}^{\prime} \backslash F^{\prime}\right)$. From now on we assume that the fibres of $\hat{E}$ have this almost complex structure $J_{F^{\prime}, 1}$ with this cylindrical end.

A neighbourhood of $\partial F^{\prime \prime}$ in $F^{\prime}$ is symplectomorphic to $L:=(-\epsilon, \epsilon) \times \partial F^{\prime \prime}$ with the symplectic form $d\left(r \alpha^{\prime \prime}\right)$. Here, $r$ is a coordinate in $(-\epsilon, \epsilon)$ and $\alpha^{\prime \prime}$ is the contact form for $\partial F^{\prime \prime}$. We also choose $\epsilon$ small enough so that $L$ is disjoint from the support of the parallel transport maps in $F^{\prime}$. Let $\bar{F}^{\prime \prime}:=F^{\prime \prime} \backslash\left((-\epsilon / 3,0] \times \partial F^{\prime \prime}\right)$. We can choose an almost complex structure $J^{\prime} \in \mathcal{J}\left(\widehat{F}^{\prime}\right)$ with the following properties:

(1) There exists a $\delta>0$ such that any holomorphic curve meeting both boundaries of $[-\epsilon,-\epsilon / 2] \times \partial F^{\prime \prime}$ has area greater than $\delta$. This is true by the monotonicity lemma [21, Lemma 1].

(2) $J^{\prime}=J_{F^{\prime}, 1}$ on $\widehat{F}^{\prime} \backslash \bar{F}^{\prime \prime}$. This means that any curve (cylinder or pair of pants) satisfying Floer's equations with respect to $H_{F^{\prime}}$ and $J^{\prime}$ with boundary in $F^{\prime \prime}$ is contained entirely in $F^{\prime \prime}$.

Construct an almost complex structure $J$ on $\widehat{E^{\prime}}$ as follows: The parallel transport maps on $\widehat{F}^{\prime} \backslash \overline{F^{\prime \prime}}$ are trivial, hence there is a region $W$ of $\widehat{E^{\prime}}$ symplectomorphic to $\mathbb{C} \times\left(\widehat{F}^{\prime} \backslash \overline{F^{\prime \prime}}\right)$. We set $\left.J\right|_{W}$ to be the product almost complex structure $J_{\mathbb{C}} \times J^{\prime}$ where $J_{\mathbb{C}}$ is the standard complex structure on $\mathbb{C}$. We then extend $\left.J\right|_{W}$ to some $J$ compatible with the symplectic form $\omega^{\prime}$ such that $\pi^{\prime}$ is $J$-holomorphic outside some large compact set. Let $H$ be a Hamiltonian of the form $\pi^{*} K+\pi_{1}^{*} H_{F^{\prime}}$ where $K$ is admissible of slope $\lambda$ on the base $\mathbb{C}$ and $\pi_{1}: W \rightarrow \widehat{F}^{\prime} \backslash \bar{F}^{\prime \prime}$ is the natural projection map. $J$ has the following properties:

(1) Any curve $u$ satisfying Floer's equations with respect to $H$ meeting both boundaries of $\left([-\epsilon,-\epsilon / 2] \times \partial F^{\prime \prime}\right) \times \mathbb{C} \subset \widehat{E^{\prime}}$ must have energy $\geq \delta$. $(u$ can be a cylinder or a pair of pants).

(2) Any such $u$ connecting orbits inside $E^{\prime \prime}$ must be entirely contained in $E^{\prime \prime}$.

Property (2) is true because: Let $u$ be a curve satisfying Floer's equations connecting orbits in $E^{\prime \prime}$, then composing $\left.u\right|_{u^{-1}(W)}$ with the natural projection $W \rightarrow \widehat{F}^{\prime} \backslash \bar{F}^{\prime \prime}$ gives 
us a curve $w$ with boundary in $F^{\prime \prime}$. This means that $w$ is contained in $F^{\prime \prime}$, and hence $u$ is contained in $E^{\prime \prime}$. Also if $u$ meets both boundaries of $\left([-\epsilon,-\epsilon / 2] \times \partial F^{\prime \prime}\right) \times \mathbb{C} \subset \widehat{E^{\prime}}$, then the projected curve $w$ has energy $\geq \delta$ which means that $u$ has energy $\geq \delta$. Hence Property (1) is true.

We perturb $H_{F^{\prime}}: \widehat{F}^{\prime} \rightarrow \mathbb{R}$ slightly so that:

(1) It is equal to 0 in $F^{\prime \prime}$.

(2) The only periodic orbits of $H_{F^{\prime}}$ are constant orbits.

(3) The action spectrum of $\left.H\right|_{\widehat{F}^{\prime} \backslash F^{\prime \prime}}$ is discrete and injective and contained in $(-\delta / 4, \delta / 4)$.

(4) We leave $H_{F^{\prime}}$ alone on the cylindrical end.

(5) All the orbits in $\widehat{F}^{\prime} \backslash F^{\prime \prime}$ are of negative action and nondegenerate.

(6) we can ensure that $H_{F^{\prime}}$ has very small positive slope with respect to the cylindrical end of $F^{\prime \prime}$ on the region $(\epsilon / 2, \epsilon) \times \partial F^{\prime \prime} \subset F^{\prime}$.

Let $\delta_{1}>0$ be the smallest distance between 0 and the action value of an orbit of $H_{F^{\prime}}$ of negative action. Here we fix some integer $m>0$. We can assume that the critical points of our Lefschetz fibration in $\mathbb{C}$ form a regular polygon with centre the origin. Draw a straight line from the origin to each critical point and let $G$ be the union of these lines. Let $X:=\frac{r}{2} \frac{\partial}{\partial r}$ be an outward pointing Liouville flow. We choose a loop $l$ around $G$ so that the disc $V$ with $\partial V=l$ has volume $v$ where $v$ can be chosen arbitrarily small, and such that $X$ is transverse to this loop. This forms a new cylindrical end $\varrho$ for $\mathbb{C}$. Now let $H_{\lambda}^{V}$ be a Hamiltonian on $V$ with slope $\lambda$. We assume that $H_{\lambda}^{V}$ has the following properties:

(1) All the orbits are nondegenerate.

(2) The action of any orbit is in the region $[0,2 v \lambda]$.

(3) All orbits of index $\leq m+n$ are exact. ( $2 n$ is the dimension of our symplectic manifold.)

(4) $2 v \lambda<\min \left(\delta / 2, \delta_{1}\right)$.

(5) $H_{\lambda}^{V}$ is constructed in the same way as the Hamiltonian $H_{S}$ mentioned before Lemma 5.6, but we do this with respect to the new cylindrical end $\varrho$ of $\mathbb{C}$.

We let $K_{\lambda}=\pi^{*}\left(H_{\lambda}^{V}\right)+\pi_{1}^{*} H_{F^{\prime}}$. Let $B:=\mathbb{C} \times\left(\widehat{F}^{\prime} \backslash F^{\prime \prime}\right) \subset \widehat{E^{\prime}}$. The Hamiltonian $K_{\lambda}$ is of the form $\pi^{*}\left(H_{\lambda}^{V}\right)+\pi_{1}^{*} H_{F^{\prime \prime}}$ on $B$. Hence the orbits on $B$ come in pairs $(\gamma, \Gamma)$, where $\gamma$ corresponds to a periodic orbit of $H_{\lambda}^{V}$ and $\Gamma$ is a constant periodic orbit of $H_{F^{\prime \prime}}$. The action difference between two orbits is $\leq \delta$ due to property (3) 
for $H_{F^{\prime}}$ and properties (2) and (4) for $H_{\lambda}^{V}$. This implies that any Floer trajectory connecting orbits $\left(\gamma_{1}, \Gamma_{1}\right)$ and $\left(\gamma_{2}, \Gamma_{2}\right)$ inside $B$ must stay inside $B$, due to property (1) from the properties of $J$. Also the definition of $\delta_{1}$ combined with properties (2) and (4) for $H_{\lambda}^{V}$ imply that all orbits inside $B$ have negative action.

Hence we have a subcomplex $C_{B}^{\lambda}$ generated by orbits in $B$. The Hamiltonian $K_{\lambda}$ in the region $\hat{E} \backslash B$ is equal to $\pi^{*}\left(H_{\lambda}^{V}\right)$. Hence, using Lemma 5.6, we have that all the orbits of $\pi^{*}\left(H_{\lambda}^{V}\right)$ have nonnegative action. This means that we have a quotient complex $C_{E^{\prime \prime}}^{\lambda}:=C_{E^{\prime}}^{\lambda} / C_{B}^{\lambda}$ where $C_{E^{\prime}}^{\lambda}$ is the complex generated by all orbits. By property (6) of $H_{F^{\prime}}$ and property (2) of $J$ we have that the limit of $H_{*}\left(C_{E^{\prime \prime}}^{\lambda}\right)$ as $\lambda$ tends to infinity is isomorphic to $\mathrm{SH}_{*}^{\text {lef }}\left(E^{\prime \prime}\right)$. So in order to show that $\operatorname{SH}_{*}^{\text {lef }}\left(E^{\prime \prime}\right)$ is isomorphic to $\mathrm{SH}_{*}^{\text {lef }}\left(E^{\prime}\right)$ we need to show that the homology of $C_{B}^{\lambda}$ is zero in all degrees $\leq m$. This would imply that $\operatorname{SH}_{*}^{\text {lef }}\left(E^{\prime \prime}\right)=\operatorname{SH}_{*}^{\text {lef }}\left(E^{\prime}\right)$ in all degrees $<m$. As $\lambda$ increases, we can make $m$ increase with $\lambda$, and this means that $\operatorname{SH}_{*}^{\text {lef }}\left(E^{\prime \prime}\right)=\operatorname{SH}_{*}^{\text {lef }}\left(E^{\prime}\right)$ in all degrees. The point is that all the orbits in $C_{B}^{\lambda}$ have negative action, so any Floer trajectory starting at one of these orbits must also finish at one of these orbits. Also, any Floer trajectory connecting two of these orbits must be contained in $B$. We have that $B$ is a product. We can assume that it has the product almost complex structure. This means that Floer trajectories between $\left(\gamma_{1}, \Gamma_{1}\right)$ and $\left(\gamma_{2}, \Gamma_{2}\right)$ come in pairs $(u, U)$ where $u$ is a Floer trajectory in $\mathbb{C}$ connecting $\gamma_{1}$ and $\gamma_{2}$ and $U$ is a Floer trajectory connecting $\Gamma_{1}$ and $\Gamma_{2}$. Hence by a Künneth formula, we have that the homology of $C_{B}^{\lambda}$ is isomorphic to $\mathrm{SH}_{*}\left(H_{\lambda}^{V}\right) \otimes \mathrm{SH}_{*}^{(-\infty, 0)}\left(H_{F^{\prime}}\right)$, where $\mathrm{SH}_{*}^{(-\infty, 0)}\left(H_{F^{\prime}}\right)$ is the subcomplex generated by orbits negative action. We have that in all degrees $\leq m$, that $\mathrm{SH}_{*}\left(H_{\lambda}^{V}\right)=0$, and that $\mathrm{SH}_{*}^{(-\infty, 0)}\left(H_{F^{\prime}}\right)$ is 0 in negative degrees which means that $\mathrm{SH}_{*}\left(H_{\lambda}^{V}\right) \otimes \mathrm{SH}_{*}^{(-\infty, 0)}\left(H_{F^{\prime}}\right)$ is zero in all degrees $\leq m$. This in turn implies that the homology of $C_{B}^{\lambda}$ is zero in all degrees $\leq m$. This gives us our isomorphism

$$
\mathrm{SH}_{*}^{\text {lef }}\left(E^{\prime \prime}\right) \cong \mathrm{SH}_{*}^{\text {lef }}\left(E^{\prime}\right) \text {. }
$$

\section{Brieskorn spheres}

In this section we will mainly be studying the variety $V$ as constructed in Section 3.1 and also the variety $M^{\prime \prime}:=\mathbb{C}^{4} \backslash V$. The variety $V$ is equal to $\left\{z_{0}^{7}+z_{1}^{2}+z_{2}^{2}+z_{3}^{2}=0\right\} \subset \mathbb{C}^{4}$.

\subsection{Parallel transport}

There is a natural symplectic form on $\mathbb{C}^{4}$ (induced from an ample line bundle on its compactification $\mathbb{P}^{4}$ ). We have a holomorphic map $P:=z_{0}^{7}+z_{1}^{2}+z_{2}^{2}+z_{3}^{2}$ with one singular point at 0 . We can view $P$ as a fibration which is compatible with this 
symplectic form as in Definition 2.11. These fibrations have a natural connection which is induced from the symplectically orthogonal plane fields to the fibres. We prove:

Theorem 7.1 Parallel transport maps are well defined for $P$.

Proof We first of all compactify $\mathbb{C}^{4}$ to $\mathbb{P}^{4}$. We let $P^{\prime}$ be a holomorphic section of $E:=\int_{\mathcal{P}} 4(7)$ :

$$
P^{\prime}\left(\left[z_{0}: \cdots: z_{4}\right]\right):=z_{0}^{7}+z_{4}^{5} z_{1}^{2}+z_{4}^{5} z_{2}^{2}+z_{4}^{5} z_{3}^{2} .
$$

This is equal to $P$ on the trivialisation $z_{4}=1$. We also have another section $Q$ defined by

$$
Q\left(\left[z_{0}: \cdots: z_{4}\right]\right):=z_{4}^{7} .
$$

The map $P$ can be extended to a rational map $P^{\prime \prime}: \mathbb{P}^{4} \rightarrow \mathbb{P}^{1}$, where $P^{\prime \prime}=P^{\prime} / Q$. Fix an identification $\mathbb{C}^{4}=\mathbb{P}^{4} \backslash Q^{-1}(0)$. We now have that

$$
P=\frac{P^{\prime}}{Q} \text {. }
$$

Let $\|\cdot\|_{E}$ be a positive curvature metric on the ample bundle $E$. We have a symplectic structure and Kähler form defined in terms of the plurisubharmonic function

$$
\phi=-\log \|Q\|_{E}^{2} .
$$

In order to show that $P$ has well defined parallel transport maps we need to construct bounds on derivatives similar to the main theorem in [15, Section 2]. We take the vector field $\partial_{z}$ on the base $\mathbb{C}$. It has a unique lift with respect to the Kähler metric which is

$$
\xi:=\frac{\nabla P}{\|\nabla P\|^{2}}
$$

Here $\|\cdot\|$ is the Kähler metric and $\nabla P$ is the gradient of $P$ with respect to this metric. Take a point $p$ on $D:=\left\{z_{4}=0\right\}$. We can assume without loss of generality that this lies in the chart $\left\{z_{1}=1\right\}$. In this chart we have that the metric $\|\cdot\|_{E}=e^{\sigma}|\cdot|$ where $\sigma$ is a smooth function and $|\cdot|$ is the standard Euclidean metric with respect to this chart. Then

$$
\phi=-\log \|Q\|^{2}=-\log |Q|^{2}-\sigma .
$$

The notation $\lesssim$ means that one term is less than or equal to some constant times the other term. Hence we get:

$$
B:=|\xi \cdot \phi| \leq \frac{|\langle\nabla P, \nabla \sigma\rangle|}{\|\nabla P\|^{2}}+\frac{2|Q| \cdot|\langle\nabla Q, \nabla P\rangle|}{\|\nabla P\|^{2} \cdot|Q|^{2}} \lesssim \frac{1}{\|\nabla P\|}+\frac{\|\nabla Q\|}{\|\nabla P\| \cdot|Q|}
$$

We get similar equations to (3) in the other charts $\left\{z_{i}=1\right\}$. If we can show that for any compact set $T \subset \mathbb{C}$ the function $B$ is bounded above by a constant $K$ in the region 
$T_{1}:=P^{-1}(T) \backslash A$ where $A=\left\{\left|z_{0}\right|,\left|z_{1}\right|,\left|z_{2}\right|,\left|z_{3}\right| \leq 1\right\}$, then we have well defined parallel transport maps. This is because we get similar bounds if we lift other vectors of unit length (ie $c \partial_{z}$ where $c \in U(1)$ ). Hence if we have a path, then $|\xi \cdot \phi|$ is bounded above by a constant on this path. This ensures that the transport maps do not escape to infinity. In the chart $\left\{z_{3}=1\right\}$, we have that for $1 \leq i \leq 2$,

$$
\partial_{i} P=2 z_{i} / z_{4}^{2}, \quad \partial_{0} P=7 z_{0}^{6} / z_{4}^{7} .
$$

We have the following bounds on derivatives:

$$
|\nabla Q| \lesssim \frac{|Q|}{\left|z_{4}\right|}
$$

Combining this with Equation (3) gives

$$
\begin{aligned}
B & \lesssim \frac{1+\left|z_{4}\right|}{\left|z_{4}\right|\left(\sum_{j=0, j \neq 3}^{4}\left|\partial_{i} P\right|\right)} \\
& \lesssim C:=\left(1+\left|z_{4}\right|\right) /\left(\frac{7\left|z_{0}\right|^{6}}{\left|z_{4}\right|^{6}}+\frac{2\left|z_{1}\right|}{\left|z_{4}\right|}+\frac{2\left|z_{2}\right|}{\left|z_{4}\right|}+\left|\partial_{4} P\right|\left|z_{4}\right|\right) \\
& \lesssim\left(1+\left|z_{4}\right|\right) /\left(\left|z_{0}\right|^{6} /\left|z_{4}\right|^{6}+\left|z_{1}\right| /\left|z_{4}\right|+\left|z_{2}\right| /\left|z_{4}\right|\right) .
\end{aligned}
$$

Hence on the chart $\left\{z_{4}=1\right\}$,

$$
B \lesssim\left(1+\left|z_{3}\right|^{-1}\right) /\left(\left|z_{0}\right|^{6}+\left|z_{1}\right|+\left|z_{2}\right|\right) .
$$

By symmetry we also have

$$
\begin{aligned}
& B \lesssim\left(1+\left|z_{2}\right|^{-1}\right) /\left(\left|z_{0}\right|^{6}+\left|z_{1}\right|+\left|z_{3}\right|\right), \\
& B \lesssim\left(1+\left|z_{1}\right|^{-1}\right) /\left(\left|z_{0}\right|^{6}+\left|z_{2}\right|+\left|z_{3}\right|\right) .
\end{aligned}
$$

In the chart $\left\{z_{0}=1\right\}$ we have

$$
B \lesssim \frac{1+\left|z_{4}\right|}{\left|z_{4}\right|\left(\sum_{j=1}^{4}\left|\partial_{i} P\right|\right)} \lesssim\left(1+\left|z_{4}\right|\right) /\left(\frac{2\left|z_{1}\right|}{\left|z_{4}\right|}+\frac{2\left|z_{1}\right|}{\left|z_{4}\right|}+\frac{2\left|z_{2}\right|}{\left|z_{4}\right|}+\left|\partial_{4} P\right|\left|z_{4}\right|\right) .
$$

Thus

$$
B \lesssim\left(1+\left|z_{4}\right|\right)\left|z_{4}\right| /\left(\left|z_{1}\right|+\left|z_{2}\right|+\left|z_{3}\right|\right),
$$

so in the chart $\left\{z_{4}=1\right\}$, we get a bound

$$
B \lesssim\left(1+\left|z_{0}\right|^{-1}\right) /\left(\left|z_{1}\right|+\left|z_{2}\right|+\left|z_{3}\right|\right) .
$$

Suppose for a contradiction that there is a sequence of vectors $\left(z_{0}^{i}, z_{1}^{i}, z_{2}^{i}, z_{3}^{i}\right)$ lying in $T_{1}$ such that $B$ tends to infinity as $i$ tends to infinity. If (after passing to a subsequence) $z_{0}^{i}$ tends to infinity, then (7) tells us that $z_{1}^{i}, z_{2}^{i}, z_{3}^{i}$ are all bounded. But this is impossible as 
$\left(z_{0}^{i}, z_{1}^{i}, z_{2}^{i}, z_{3}^{i}\right)$ lies in $T_{1}$ which means that $z_{0}^{i}$ is bounded. Similarly, using Equations (4), (5) and (6) we get that $z_{j}^{i}$ is bounded. Hence $B$ is bounded away from the compact set $\left\{\left|z_{0}\right|,\left|z_{1}\right|,\left|z_{2}\right|,\left|z_{3}\right| \leq 1\right\}$. This means that $B$ is bounded when restricted to $T_{1}$, so we have well defined parallel transport maps.

Let $\left(\mathbb{C}^{4}, \theta\right)$ be the convex symplectic manifold induced by the compactification $\mathbb{C}^{4} \hookrightarrow$ $\mathbb{P}^{4}$. Because parallel transport maps for $P$ are well defined we can use ideas from $[26$, Section 19b] to deform the 1 -form $\theta$ on $\mathbb{C}^{4}$ through a series of 1 -forms $\theta_{t}$ such that:

(1) Each $\omega_{t}:=d \theta_{t}$ is compatible with $P$ as in Definition 2.11 and $\theta_{t}$ is a convex symplectic deformation on $\mathbb{C}^{4}$.

(2) The parallel transport maps of $P$ with respect to the connection induced by $\omega_{1}$ are trivial at infinity. This means that near infinity, $P$ looks like the natural projection $C \times \mathbb{C} \rightarrow \mathbb{C}$ where $C$ is the complement of some compact set in $V$.

(3) For a smooth fibre $F$ of $P,\left(F, \theta_{1}\right)$ is exact symplectomorphic to $\left(F, \theta_{0}\right)$.

We have $M^{\prime \prime}=\mathbb{C}^{4} \backslash P^{-1}(0)$, so we can restrict $P$ to a fibration $P^{\prime \prime}=\left.P\right|_{M^{\prime \prime}}: M^{\prime \prime} \rightarrow \mathbb{C}^{*}$. Let $\theta_{S}$ be a convex symplectic structure on $\mathbb{C}^{*}$ with the property that $\theta_{M^{\prime \prime}, t}:=$ $\left.\theta_{t}\right|_{M^{\prime \prime}}+P^{\prime \prime *} \theta_{S}$ is a convex symplectic structure for $M^{\prime \prime}$. Let $\theta^{\prime \prime}$ be a convex symplectic structure on $M^{\prime \prime}$ constructed as in Example 2.8. It is convex deformation equivalent to $\left(M^{\prime \prime}, \theta_{M^{\prime \prime}, 0}\right)$ as follows: Let $F$ be a fibre of $P^{\prime \prime}$, then $\left(F,\left.\theta_{M^{\prime \prime}, 0}\right|_{F}\right)$ is convex deformation equivalent to $\left(F,\left.\theta^{\prime \prime}\right|_{F}\right)$ by Lemma 2.9 as both convex structures come from Stein structures constructed algebraically as in Example 2.8. This deformation is $\left.(1-t) \theta_{M^{\prime \prime}, 0}\right|_{F}+\left.t \theta^{\prime \prime}\right|_{F}$. The following family of 1 -forms $\Theta_{t}:=(1-t) \theta_{M^{\prime \prime}, 0}+t \theta^{\prime \prime}$ induces a convex symplectic deformation (we might have to add $\pi^{*} \theta_{S}^{\prime}$ to $\theta_{M^{\prime \prime}, 0}$ and $\theta^{\prime \prime}$ where $\theta_{S}$ is a convex symplectic structure on $\mathbb{C}^{*}$ and $d \theta_{S}$ is sufficiently large). The reason why it is a convex symplectic deformation is as follows: We can ensure that $\theta_{S}$ comes from a Stein function $\phi_{S}$ on $\mathbb{C}^{*}$. Also, $\theta_{0}$ comes from some Stein function $\phi: \mathbb{C}^{4} \rightarrow \mathbb{R}$, hence $\theta_{M^{\prime \prime}, 0}$ comes from a Stein function $\phi_{0}:=\left.\phi\right|_{M^{\prime \prime}}+P^{\prime \prime *} \phi_{S}$. The 1 -form $\theta^{\prime \prime}$ comes from a Stein function $\phi_{1}$. Hence $\Theta_{t}$ comes from a Stein function of the form $\phi_{t}:=(1-t) \phi_{0}+t \phi_{1}$. The set of singular points of $\left.\phi_{t}\right|_{F}$ for all $t$ lie inside a compact set $K_{F}$ (independent of $t$ ) for each fibre $F$. Let $K$ be the union of all the compact sets $K_{F}$ for each fibre $F$ in $M^{\prime \prime}$. We can choose $\theta_{S}$ large enough so that outside some annulus $A$ in $\mathbb{C}^{*}, \phi_{t}$ has no singularities in $K \cap P^{\prime \prime-1}\left(\mathbb{C}^{*} \backslash A\right)$. Also, there are no singularities of $\phi_{t}$ outside $K$. Hence, all the singularities of $\phi_{t}$ stay inside some compact set independent of $t$. This means that $\phi_{t}$ is a Stein deformation. This means that $\left(M^{\prime \prime}, \theta_{M^{\prime \prime}, 1}\right)$ is convex deformation equivalent to $\left(M^{\prime \prime}, \theta_{M^{\prime \prime}, 0}\right)$ which is convex deformation equivalent to $\left(M^{\prime \prime}, \theta^{\prime \prime}\right)$.

Hence on $\left(M^{\prime \prime}, \theta^{\prime \prime}\right)$, we have that the parallel transport maps of $P^{\prime \prime}$ are trivial at infinity after a convex symplectic deformation to $\left(M^{\prime \prime}, \theta_{M^{\prime \prime}, 1}\right)$. 


\subsection{Indices}

Let $P^{\prime \prime}: M^{\prime \prime} \rightarrow \mathbb{C}^{*}, P^{\prime \prime}(z)=P(z)$. Let $F$ be a smooth fibre of $P^{\prime \prime}$. This fibre has a natural exhausting plurisubharmonic function $\phi$ as in Example 2.8. We can modify $\phi$ to an exhausting plurisubharmonic function $\phi^{\prime}$ which is complete by [27, Lemma 6]. We denote this new Stein manifold by $\widehat{F}$. The following theorem is about indices of a cofinal family of Hamiltonians on $\widehat{F}$.

Theorem 7.2 There is a cofinal family of Hamiltonians $H_{\lambda}$ on $\widehat{F}$ with the following properties:

(1) There exists some convex symplectic submanifold $T$ of $F$ such that $\hat{T}$ (the symplectic completion of $T$ ) is exact symplectomorphic to $\widehat{F}$.

(2) $H_{\lambda}=0$ on $T$.

(3) If $y$ is a periodic orbit of $H_{\lambda}$ not in $T$ then $\operatorname{ind}(y) \geq 2$.

(4) For each $k \in \mathbb{Z}$ there exists an $N>0$ (independent of $\lambda$ ) such that the number of periodic orbits of $H_{\lambda}$ of index $k$ is bounded above by $N$.

(5) If we don't count critical points from the interior, then there is exactly one orbit of index 2 and one orbit of index 3 such that the action difference between these two orbits tends to 0 as $\lambda$ tends to infinity. Also the number of Floer cylinders connecting these orbits is even.

This theorem is proved by analysing the Conley-Zehnder indices of a Reeb foliation on the Brieskorn sphere $V \cap S$, where $S$ is the unit sphere in $\mathbb{C}^{4}$. This result needs the following two lemmas:

Lemma 7.3 $\hat{F}$ is the completion of some convex symplectic submanifold $T$ with boundary the Brieskorn sphere $V \cap S$.

Proof By Theorem 7.1, we have that $V \backslash 0$ is symplectomorphic to $F \backslash K$ where $K$ is a compact set. Hence there exists a cylindrical end of $\widehat{F}$ which is symplectomorphic to the cylindrical end of $V$ induced by flowing $V \cap S$ by parallel transport.

Lemma 7.4 There is a contact form on the Brieskorn sphere $V \cap S$ such that all the $R e e b$ orbits are nondegenerate and they have Conley-Zehnder indices $\geq 2$. Also, there is exactly one orbit of index 2 and no orbits of index 3 and finitely many orbits of degree $k$ for each $k$. 
Proof In [28] Ustilovsky constructs a contact form such that all the Reeb orbits are nondegenerate and such that their reduced Conley-Zehnder index is $\geq 2(n-2)$ where $n=3$ in our case. Ustilovsky defines the reduced Conley-Zehnder index to be equal to the Conley-Zehnder index $+(n-3)$. This means that the Reeb orbits have ConleyZehnder index $\geq n-1=2$. He also shows for each $k \in \mathbb{Z}$, there are finitely many orbits of Conley-Zehnder index $k$. He shows that there are no orbits of odd index and the orbit of lowest index has index 2 .

Proof of Theorem 7.2 By Lemma 7.3 $\hat{F}$ has a convex cylindrical end which is symplectomorphic to $[1, \infty) \times \Sigma$ where $\Sigma$ is the Brieskorn sphere $V \cap S$. We choose a Hamiltonian which is constant on the interior of $F$ and equal to $h(r)$ on the cylindrical end, where $r$ parameterizes $[1, \infty)$. We also assume that $h^{\prime}(r)$ is constant and not in the period spectrum of $B$ at infinity. Also, near each orbit in the cylindrical end, we assume that $h^{\prime \prime}>0$. The flow of the Hamiltonian at the level $r=k$ is the same as the flow of $X_{H}:=-h^{\prime}(k) R$, where $R$ is the Reeb flow. The Conley-Zehnder indices from Lemma 7.4 are computed by trivialising the contact plane bundle. We can trivialise the symplectic bundle by first trivialising the contact plane bundle and then trivialising its orthogonal bundle. We trivialise the orthogonal bundle by giving it a basis $\left(\frac{\partial}{\partial r}, R\right)$. The symplectic form restricted to this basis is the standard form

$$
\left(\begin{array}{cc}
0 & 1 \\
-1 & 0
\end{array}\right)
$$

The Hamiltonian flow in this trivialisation is the matrix

$$
\left(\begin{array}{cc}
1 & 0 \\
h^{\prime \prime} t & 1
\end{array}\right)
$$

along the orthogonal bundle. This is because $R$ is invariant under this flow and the Lie bracket of $X_{H}=-h^{\prime} R$ with $\frac{\partial}{\partial r}$ is $h^{\prime \prime} R$. The Robbin-Salamon index of this family of matrices is $\frac{1}{2}$. We calculate this index by perturbing this family of matrices by a function $\xi:[0,1] \rightarrow \mathbb{R}$ where $\xi(0)=\xi(1)=0$ as follows:

$$
\left(\begin{array}{cc}
1 & \xi(t) \\
h^{\prime \prime} t & 1
\end{array}\right)
$$

Choosing $\xi$ so that its derivative is nonzero whenever $\xi=0$ ensures that the path is generic enough to enable us to compute its Robbin-Salamon index.

Remember that the Robbin-Salamon index of an orbit is equal to the Conley-Zehnder index taken with negative sign. Lemma 7.4 tells us the Conley-Zehnder indices of all the Reeb orbits. The flow $X_{H}=-h^{\prime} R$ of the Hamiltonian has orbits in the opposite direction to Reeb orbits. Hence the Robbin-Salamon index (restricted to the contact 
plane field) of an orbit of $X_{H}=-h^{\prime} R$ is the same as the Conley-Zehnder index of the corresponding Reeb orbit. Hence the Robbin-Salamon index of some orbit of the Hamiltonian on the level set $r=k$ is equal to $C+\frac{1}{2}$ where $C$ is the Conley-Zehnder index of the associated Reeb orbit as calculated in Lemma 7.4. Hence the indices of these orbits are $\geq 2+\frac{1}{2}$.

The problem is that these orbits are degenerate. This is why their index is not an integer. As in [20, Section 3] we can perturb each circle of orbits to a pair of nondegenerate orbits. Let $C^{\prime}$ be a circle of orbits. We choose a Morse function $f$ on $C^{\prime}$. If we flow $f$ along $X_{H}$ (the Hamiltonian flow of $H$ ) we get a time dependent Morse function $f_{t}=f \circ \phi_{-t}\left(\phi_{t}\right.$ is the Hamiltonian flow). Extend $f_{t}$ so that it is defined as a function on a neighbourhood of $C^{\prime}$. Let $H+f_{t}$ be our new Hamiltonian. The orbits near $C^{\prime}$ now correspond to critical points $p$ of $f$. The Robbin-Salamon index of such an orbit is

$$
i\left(C^{\prime}\right)+\frac{1}{2} \operatorname{sign}\left(\nabla_{p}^{2} f\right)
$$

where $i\left(C^{\prime}\right)$ is the Robbin-Salamon index of the manifold of orbits. The symbol "sign" means the number of positive eigenvalues minus the number of negative eigenvalues. In our case we can choose $f$ so that it has 2 critical points $p_{1}, p_{2}$ such that

$$
\operatorname{sign}\left(\nabla_{p_{1}}^{2} f\right)=1, \operatorname{sign}\left(\nabla_{p_{2}}^{2} f\right)=-1 .
$$

Hence, if the Conley-Zehnder index of a Reeb orbit $C$ is $k$, then we can perturb $H$ so that the associated Hamiltonian orbits have Robbin-Salamon index (or equivalently Conley-Zehnder index taken with negative sign) $k+0$ and $k+1$. This means all the nonconstant orbits of $H$ have Robbin-Salamon index $\geq 2$.

We now need to show that there are a finite number of orbits in each degree. This follows directly from Lemma 7.4 which says that there are finitely many Reeb orbits in each degree. Finally this same lemma says that there is only one Reeb orbit with Conley-Zehnder index 2 and no Reeb orbits with Conley-Zehnder index 3. So the Hamiltonian $H$ has one orbit of Robbin-Salamon index 2 and one orbit of index 3 . We can also ensure that the actions of these orbits are arbitrarily close by letting the associated Morse function $f$ be $C^{2}$ small. There are an even number of Floer cylinders connecting the orbit of index 3 with the orbit of index 2 by [9, Proposition 2.2].

Lemma 7.5 We have $H^{i}\left(M^{\prime \prime}\right)=0$ for $i \geq 2$.

Proof $M^{\prime \prime}=\mathbb{C}^{4} \backslash V$. Theorem 3.1 tells us that $V$ is homeomorphic to $\mathbb{R}^{6}$. This means that there is a neighbourhood $B$ of $V$ which retracts onto $V$ whose boundary $\partial B$ satisfies $H^{i}(\partial B)=0$ for $i \geq 2$. The Mayor-Vietoris sequence involving $B, M^{\prime \prime}$ and $B \cup M^{\prime \prime}=\mathbb{C}^{4}$ ensures that $H^{i}\left(M^{\prime \prime}\right)=0$ for $i \geq 2$. 


\subsection{Symplectic homology of these varieties}

We wish to show that the symplectic homology of the variety $M^{\prime \prime}:=\mathbb{C}^{4} \backslash V$ has only finitely many idempotents using the results of the previous two sections. We will then show that it has at least two idempotents: 0 and 1 . First of all we need the following lemma: We let $R$ be an algebra over $\mathbb{Z} / 2 \mathbb{Z}$ which is graded by a finitely generated abelian group $G$. This means that as a vector space, $R=\bigoplus_{g \in G} R_{g}$ with the property that if $a \in R_{g_{1}}$ and $b \in R_{g_{2}}$ then the product $a b$ is contained in $R_{g_{1}} \cdot g_{2}$.

Lemma 7.6 If $a$ is an idempotent in $R$ then $a \in \bigoplus_{g \in G_{n}} R_{g}$ where $G_{n}$ is the subgroup of torsion elements of $G$.

Proof We have $a=a_{g_{1}}+\cdots+a_{g_{n}}$ where $g_{i} \in G$ and $a_{g_{i}} \in R_{g_{i}}$. Suppose for a contradiction we have that $a=a^{2}$ and $g_{1}$ is not torsion. Then $a^{2}=a_{g_{1}}^{2}+\cdots+a_{g_{n}}^{2}$. The group $G / G_{n}$ is a free $\mathbb{Z}$ algebra, hence there is a group homomorphism $p: G \rightarrow$ $G / G_{n} \rightarrow \mathbb{Z}$ such that $p\left(g_{1}\right) \neq 0$. The map $p$ gives $R$ a $\mathbb{Z}$ grading. Let $b$ be an element of $R$. It can be written uniquely as $b=b_{1}+\cdots+b_{k}$ where $b_{i}$ are nonzero elements of $R$ with grading $q_{i} \in \mathbb{Z}$. We can define $f(b)$ as $\min \left\{\left|q_{j}\right| \neq 0\right\}$. Note that $f(b)$ is well defined only if at least one of the $q_{i}$ 's are nonzero. Because $p\left(g_{1}\right) \neq 0$, we have that $f(a)$ is well defined and positive. We also have that $f\left(a^{2}\right) \geq 2 f(a)$ which means that $a^{2} \neq a$. This contradicts the fact that $a$ is an idempotent.

The vector space $\mathrm{SH}_{4+*}\left(M^{\prime \prime}\right)$ is a ring bigraded by the Robbin-Salamon index and the first homology group. We write $4+*$ here because the unit has Robbin-Salamon index 4 . The previous lemma shows us that any idempotent must have grading 4 in $\mathrm{SH}_{*}\left(M^{\prime \prime}\right)$ and be in a torsion homology class.

We have a map $P^{\prime \prime}: M^{\prime \prime} \rightarrow \mathbb{C}^{*}$. At the end of Section 7.1 we had a convex symplectic structure $\left(M^{\prime \prime}, \theta_{M^{\prime \prime}, 1}\right)$. Let $A$ be a large annulus in the base $\mathbb{C}^{*}$ which is a compact convex symplectic manifold. Let $\left(F^{\prime \prime}, \theta_{M^{\prime \prime}, 1}\right)$ be a fibre of $P^{\prime \prime}$. Choose a compact convex symplectic manifold (with corners) $\bar{M}^{\prime \prime}$ such that $\left(\bar{M}^{\prime \prime}, \bar{P}^{\prime \prime}:=P^{\prime \prime} \mid \bar{M}^{\prime \prime}, \theta_{M^{\prime \prime}, 1}\right)$ is a compact convex Lefschetz fibration with fibres $\bar{F}^{\prime \prime}$ and base $A \subset \mathbb{C}^{*}$. We can also ensure that $\partial \bar{F}^{\prime \prime}$ is transverse to $\lambda_{1}$ (the associated Liouville vector field of $\mathbb{F}^{\prime \prime}$ ) and there are no singularities of $\lambda_{1}$ outside $\bar{F}^{\prime \prime}$ in $F^{\prime \prime}$. So the completion of $\bar{M}^{\prime \prime}$ is $\widehat{M}^{\prime \prime}$. Let $\left(\widehat{E}^{\prime \prime}, \pi^{\prime \prime}\right)$ be the completion of $\left(\bar{M}^{\prime \prime}, \bar{P}^{\prime \prime}, \theta_{M^{\prime \prime}, 1}\right)$ (so that $\widehat{\bar{M}}^{\prime \prime}=\widehat{E^{\prime \prime}}$ ). We wish to use the results of Section 5 to show that $\mathrm{SH}_{*}\left(E^{\prime \prime}\right)$ has finitely many idempotents, and hence $\mathrm{SH}_{*}\left(M^{\prime \prime}\right)$ has finitely many idempotents. Let $H$ be a Lefschetz admissible Hamiltonian for $\widehat{E^{\prime \prime}}$. Let $C$ be the cylindrical end of $\widehat{F^{\prime \prime}}$. We may assume that this cylindrical end is of the form $\left(S_{V} \times[1, \infty), r_{F} \alpha_{F}\right)$ where $\left(S_{V}, \alpha_{F}\right)$ is the Brieskorn 
sphere described in Section 7.2 and $r_{F}$ is the coordinate for $[1, \infty)$. The Hamiltonian $H$ is of the form $\pi^{\prime \prime *} H_{S^{\prime \prime}}+\pi_{1}^{\prime \prime *} H_{F^{\prime \prime}}$ as in Definition 2.21. By Lemma 7.6, we have that any idempotent must come from a linear combination of orbits of $H$ in torsion homology classes as long as $H$ is large enough (ie it is large enough in some cofinal sequence of Lefschetz admissible Hamiltonians). Away from $C \times \mathbb{C}^{*} \subset \widehat{E^{\prime \prime}}$ we have that the Hamiltonian flow of $H$ is the same as the flow of $L:=\pi^{\prime \prime *} H_{S^{\prime \prime}}$. Let $X$ be the Hamiltonian vector field associated to $L$, and let $X_{S^{\prime \prime}}$ be the Hamiltonian vector field in $\mathbb{C}^{*}$ associated to $H_{S^{\prime \prime}}$. Then the value of $X$ at a point $p$ is some positive multiple of the horizontal lift of $X_{S^{\prime \prime}}$ to the point $p$. We can assume that $H_{S^{\prime \prime}}$ has exactly two contractible periodic orbits of index 0 and 1 corresponding to Morse critical points of $H_{S^{\prime \prime}}$ (as any Reeb orbit of $\mathbb{C}^{*}$ is not contractible). We can also make $H_{S^{\prime \prime}} C^{2}$ small away from the cylindrical ends of $\mathbb{C}^{*}$ so that the only Floer cylinders connecting contractible orbits correspond to Morse flow lines. Hence, any contractible orbit of $X$ must project down to a constant orbit of $X_{S^{\prime \prime}}$. We let $H_{F^{\prime \prime}}$ be a Hamiltonian as in Theorem 7.2 above in Section 7.2. We let our almost complex structure $J$ when restricted to $C \times \mathbb{C}^{*} \subset \widehat{E^{\prime \prime}}$ be equal to the product almost complex structure $J_{F} \times J_{\mathbb{C}^{*}}$ where $J_{F}$ is an admissible almost complex structure on $\widehat{F}$ and $J_{\mathbb{C}^{*}}$ is the standard complex structure on $\mathbb{C}^{*}$. The contractible orbits in this cylindrical end come in pairs $(\Gamma, \gamma)$ where $\Gamma$ is an orbit in $\hat{F}$ and $\gamma$ is a contractible orbit in $\mathbb{C}^{*}$. Because there are only 2 contractible orbits in $\mathbb{C}^{*}$ and there are finitely many orbits in each degree in $\widehat{F}$, we have finitely many contractible orbits of index 4 for $H$. Hence:

\section{Theorem 7.7 The ring $\mathrm{SH}_{4+*}\left(M^{\prime \prime}\right)$ has only finitely many idempotents.}

We now wish to show that $\mathrm{SH}_{*}\left(M^{\prime \prime}\right)$ has at least 2 idempotents. To do this we show that $\mathrm{SH}_{*}\left(M^{\prime \prime}\right) \neq 0$, and hence has a unit by [25, Section 8]. This means that $\mathrm{SH}_{*}\left(M^{\prime \prime}\right)$ has 0 and 1 as idempotents. The Hamiltonian $H$ has nondegenerate orbits in $C \times \mathbb{C}^{*} \subset \widehat{E^{\prime \prime}}$, so we perturb $H$ away from this set to make all its orbits nondegenerate. In $E^{\prime \prime} \subset \widehat{E^{\prime \prime}}$ we can ensure that $H$ is $C^{2}$ small and $J$ is independent of $t$, hence the only orbits in this region are critical points of $H$ and the only Floer cylinders correspond to Morse flow lines. The orbits corresponding to critical points of $H$ have Robbin-Salamon index $\geq 3$ because $H^{i}\left(M^{\prime \prime}\right)=0$ for $i>1$ by Lemma 7.5. Hence all orbits have index $\geq 2$. There is only one orbit of index 2 . This orbit is in the cylindrical end $C \times \mathbb{C}^{*} \subset \widehat{E^{\prime \prime}}$. Hence the orbit is of the form $\left(\Gamma_{m}, \gamma_{m}\right)$ where $\gamma_{m}$ has index 0 and $\Gamma_{m}$ has index 2 . This orbit is closed because there are no orbits of lower index. Suppose for a contradiction this orbit is exact, then there exists a Floer cylinder connecting an orbit $\beta$ of index 3 with $\left(\Gamma_{m}, \gamma_{m}\right)$. This orbit $\beta$ must be contractible, so it is either a critical point, or it is of the form $\left(\Gamma_{1}, \gamma_{1}\right)$ in $C \times \mathbb{C}^{*} \subset \widehat{E^{\prime \prime}}$. The action of $\left(\Gamma_{m}, \gamma_{m}\right)$ is larger than the action of a critical point and hence $\beta$ cannot 
be a critical point. Hence $\beta$ is of the form $\left(\Gamma_{1}, \gamma_{1}\right)$. Suppose that the index of $\Gamma_{1}$ is 3. We have $\gamma_{m}=\gamma_{1}$ and by Theorem 7.2 we can ensure that the action difference between $\Gamma_{1}$ is arbitrarily close to $\Gamma_{m}$. Similarly if $\gamma_{1}$ has index 1 then we can ensure that $\Gamma_{m}=\Gamma_{1}$ and the action difference between $\gamma_{m}$ and $\gamma_{1}$ is arbitrarily small. This means that the action difference between $\left(\Gamma_{m}, \gamma_{m}\right)$ and $\left(\Gamma_{1}, \gamma_{1}\right)$ is arbitrarily small. This means that if we have a Floer cylinder connecting $\left(\Gamma_{m}, \gamma_{m}\right)$ and $\left(\Gamma_{1}, \gamma_{1}\right)$ then Gromov compactness ensures that it must stay in the region $C \times \mathbb{C}^{*} \subset \widehat{E^{\prime \prime}}$ (Because the action of $\left(\Gamma_{m}, \gamma_{m}\right)$ tends to the action of $\left(\Gamma_{1}, \gamma_{1}\right)$, we get a sequence of Floer cylinders converging to a Floer cylinder of energy 0 which cannot exit $\left.C \times \mathbb{C}^{*} \subset \widehat{E^{\prime \prime}}\right)$. Because all the Floer cylinders stay inside $C \times \mathbb{C}^{*} \subset \widehat{E^{\prime \prime}}$, the number of Floer cylinders connecting $\left(\Gamma_{m}, \gamma_{m}\right)$ and $\left(\Gamma_{1}, \gamma_{1}\right)$ is equal to the number of Floer cylinders connecting $\Gamma_{m}$ and $\Gamma_{1}$ multiplied by the number of Floer cylinders connecting $\gamma_{m}$ and $\gamma_{1}$. We need to show that the number of Floer cylinders connecting $\left(\Gamma_{1}, \gamma_{1}\right)$ and $\left(\Gamma_{m}, \gamma_{m}\right)$ is even and by the previous comment, this means we only need to show that the number of Floer cylinders connecting $\Gamma_{1}$ and $\Gamma_{m}$ is even or the number of Floer cylinders connecting $\gamma_{1}$ and $\gamma_{m}$ is even. But the number of Floer cylinders connecting $\Gamma_{1}$ and $\Gamma_{m}$ is even if $\Gamma_{1}$ has index 3 (by part (5) of Theorem 7.2) and similarly $\gamma_{1}$ is closed if it has index 1 (so there are an even number of Floer cylinders connecting $\gamma_{1}$ and $\left.\gamma_{m}\right)$. Hence the number of Floer cylinders connecting these two orbits is even and so $\left(\Gamma_{m}, \gamma_{m}\right)$ is not exact. Hence $\mathrm{SH}_{*}\left(M^{\prime \prime}\right) \neq 0$.

This completes the proof of the main Theorem 1.1 subject to checking ring addition under end connect sums.

\section{Appendix A: Lefschetz fibrations and the Kaliman modifi- cation}

Let $X, D, M$ be as in Example 2.8. This means that $X$ is a projective variety with $D$ an effective ample divisor and $M=X \backslash D$ an affine variety. Let $Z$ be an irreducible divisor in $X$ and $q \in(Z \cap M)$ a point in the smooth part of $Z$. We assume there is a rational function $m$ on $X$ which is holomorphic on $M$ such that $\overline{m^{-1}(0)}$ is reduced and irreducible and $Z=\overline{m^{-1}(0)}$. Let $M^{\prime}:=\operatorname{Kalmod}(M,(Z \cap M),\{q\})$, and let $M^{\prime \prime}:=M \backslash Z$. Suppose also that $\operatorname{dim}_{\mathbb{C}} X \geq 3$. Recall the following theorem.

Theorem 2.32 There exist Lefschetz fibrations $E^{\prime \prime} \subset E^{\prime}$ respectively satisfying the conditions of Theorem 1.3 such that $E^{\prime}$ (resp. $E^{\prime \prime}$ ) is convex deformation equivalent to $M^{\prime}$ (resp. $M^{\prime \prime}$ ). 
The rest of this section is used to prove this theorem. We will start with several preliminary lemmas.

Lemma 8.1 There are Stein functions $\phi^{\prime}$ (resp. $\phi^{\prime \prime}$ ) on $M^{\prime}$ (resp. $M^{\prime \prime}$ ) such that $M^{\prime \prime}$ becomes a symplectic submanifold of $M^{\prime}$.

Proof Let $m^{\prime}$ be the pullback of $m$ to $\mathrm{Bl}_{q} X$. Let $Z^{\prime}$ be the divisor defined by the zero set of $m^{\prime}$. Let $Z^{\prime \prime}$ be the divisor defined by the zero set of $1 / \mathrm{m}^{\prime}$, so that $Z^{\prime}$ is linearly equivalent to $Z^{\prime \prime}$. By abuse of notation, we write $D$ as the total transform of $D$ in $\mathrm{Bl}_{q} X$.

Let $\tilde{Z}$ be the proper transform of $Z$. We can choose an effective ample divisor $D^{\prime}$ with support equal to $\widetilde{Z} \cup D$ (as a set) so that $D^{\prime}-Z^{\prime \prime}$ is effective. We have that $Y_{1}:=D^{\prime}$ and $Y_{2}:=Y_{1}-Z^{\prime \prime}+Z^{\prime}$ are linearly equivalent effective ample divisors. Let $E$ be a line bundle associated to $Y_{1}$ and let $s_{1}, s_{2}$ be sections so that $s_{i}^{-1}(0)=Y_{i}$. There is a metric $\|\cdot\|$ of positive curvature on $E$. We define $\phi^{\prime}:=-d d^{c} \log \left(s_{1}\right)$ and $\phi^{\prime \prime}:=-d d^{c} \log \left(s_{2}\right)$.

Moving the point $q$ within the smooth part of $Z \cap M$ induces a Stein deformation of $M^{\prime}$ and $M^{\prime \prime}$ by a slight modification of the above lemma.

We now need a technical lemma involving convex symplectic manifolds of finite type. Let $\left(M, \theta_{1}\right),\left(M, \theta_{2}\right)$ be convex symplectic manifolds. Suppose that $\theta_{1}=\theta_{2}$ inside some codimension 0 submanifold $C$ such that $\left(C, \theta_{1}\right)$ is a compact convex symplectic manifold.

Lemma 8.2 If all the singular points of $\theta_{1}$ and $\theta_{2}$ are contained in $C$, then $\left(M, \theta_{1}\right)$ is convex deformation equivalent to $\left(M, \theta_{2}\right)$.

Proof The interior $C^{o}$ of $C$ has the structure of a finite type noncomplete convex symplectic manifold constructed as follows: The boundary of $C$ has a collar neighbourhood in $C$ of the form $N:=(-\epsilon, 1] \times \partial C$, with $\theta_{1}=r \alpha$. Here $r$ is the coordinate on $(-\epsilon, 1]$, and $\alpha$ is a contact form on $\partial C$. We let $\psi: C^{o} \rightarrow \mathbb{R}$ be an exhausting function, which is of the form $h(r)$ on $N$ and such that $h(r) \rightarrow \infty$ as $r \rightarrow 1$. For some $N \gg 0$, we have that $\psi^{-1}(l)$ is transverse to the associated Liouville field $\lambda_{1}$ for all $l \geq N$. Let $\phi_{1}$ be the function associated to the convex symplectic structure $\left(M, \theta_{1}\right)$. We may assume that $\phi_{1}^{-1}(l)$ is transverse to $\lambda_{1}$ for all $l \geq N$ as well. We can smoothly deform the function $\phi_{1}$ into the function $\psi$ through a series of exhausting functions $\phi_{t}$ (the domain of $\phi_{t}$ smoothly changes within $M$ as $t$ varies) such that $\phi_{t}^{-1}(N+k)$ is transverse to $\lambda_{1}$ for each $k \in \mathbb{N}$. This induces a convex symplectic deformation 
from $\left(M, \theta_{1}\right)$ to $\left(C^{o}, \theta_{1} \mid C^{o}\right)$. Similarly we have a convex symplectic deformation from $\left(M, \theta_{2}\right)$ to $\left(C^{o},\left.\theta_{1}\right|_{C^{o}}\right)$. Hence, $\left(M, \theta_{1}\right)$ is convex deformation equivalent to $\left(M, \theta_{2}\right)$.

We need another similar lemma about deformation equivalence.

Lemma 8.3 Suppose $\left(M, \theta_{1}\right)$ and $\left(M, \theta_{2}\right)$ are convex symplectic manifolds such that $\theta_{1}=\theta_{2}+d R$ for some function $R$, then $\left(M, \theta_{1}\right)$ is convex deformation equivalent to $\left(M, \theta_{2}\right)$.

Proof Let $\phi_{1}$ (resp. $\phi_{2}$ ) be the function associated with the convex symplectic structure $\left(M, \theta_{1}\right)$ (resp. $\left.\left(M, \theta_{2}\right)\right)$. Choose constants $c_{1}<c_{2}<\cdots$ and $d_{1}<d_{2}<\cdots$ tending to infinity such that $M_{i}^{1}:=\phi_{1}^{-1}\left(-\infty, c_{i}\right]$ (resp. $\left.M_{i}^{2}:=\phi_{2}^{-1}\left(-\infty, c_{i}\right]\right)$ are compact convex symplectic manifolds. Also we assume that

$$
M_{i}^{1} \subset M_{i}^{2} \subset M_{i+1}^{1} \subset M_{i+1}^{2}
$$

for all $i$. Let $R^{\prime}: M \rightarrow \mathbb{R}$ be a function such that $R^{\prime}=0$ on a neighbourhood of $\partial M_{i}^{1}$ and $R^{\prime}=R$ on a neighbourhood of $\partial M_{i}^{2}$ for all $i$. Let $\theta_{3}:=\theta_{1}+d R^{\prime}$. We will show that both $\left(M, \theta_{1}\right)$ and $\left(M, \theta_{2}\right)$ are convex deformation equivalent to $\left(M, \theta_{3}\right)$. Let $R_{t}: M \rightarrow \mathbb{R}$ be a family of functions such that $R_{t}=0$ on a neighbourhood of $\partial M_{i}^{1}$ for all $i$ and such that $R_{0}=0$ and $R_{1}=R^{\prime}$. Then $\left(M, \theta_{1}+d R_{t}\right)$ is a convex deformation from $\left(M, \theta_{1}\right)$ to $\left(M, \theta_{3}\right)$ because $\left(M_{i}^{1}, \theta_{1}+d R_{t}\right)$ is a compact convex symplectic manifold for all $i$. Also let $R_{t}^{\prime}: M \rightarrow \mathbb{R}$ be a family of functions such that $R_{t}^{\prime}=R$ on a neighbourhood of $\partial M_{i}^{2}$ and such that $R_{0}^{\prime}=R$ and $R_{1}^{\prime}=R^{\prime}$. Then $\left(M, \theta_{1}+d R_{t}^{\prime}\right)$ is a convex deformation from $\left(M, \theta_{2}\right)$ to $\left(M, \theta_{3}\right)$. Hence $\left(M, \theta_{1}\right)$ is convex deformation equivalent to $\left(M, \theta_{2}\right)$.

We let $E$ be an ample line bundle on $X$, and $s, t$ sections of $E$. We assume that $s$ is nonzero on $M$. Let $t$ be a holomorphic section of $E$, and let $p:=t / s$ be a map from $M$ to $\mathbb{C}$.

Definition 8.4 We call $(M, p)$ an algebraic Lefschetz fibration if:

(1) $\overline{t^{-1}(0)}$ is smooth, reduced and intersects each stratum of $D$ transversally.

(2) $p$ has only nondegenerate critical points and there is at most one of these points on each fibre.

An algebraic Lefschetz fibration $(M, p)$ has a symplectic form $\omega$ constructed as in Example 2.8. This means that $\omega$ is compatible with $p$. These are not exact Lefschetz fibrations since the horizontal boundary is not trivial, but they are very useful since our examples arise in this way. 
Theorem 8.5 Parallel transport maps for an algebraic Lefschetz fibration are well defined.

This is basically proved in [15, Section 2], but, there is a subtle distinction between the above theorem and theirs. In [15, Section 2], the Stein structure and the Lefschetz fibration are constructed from the same compactification $(X, D)$ of $M$. In our case they come from different compactifications. The proof can be easily adjusted to this case. This is due to the fact that if we have two different metrics on $M$ induced from compactifications $\left(X_{1}, D_{1}\right)$ and $\left(X_{2}, D_{2}\right)$, then the $C^{2}$ distance between them is bounded.

We need the following technical lemma so that we can relate algebraic Lefschetz fibrations with ordinary Lefschetz fibrations. We let $\left(E^{\prime}, \pi^{\prime}\right),\left(E^{\prime \prime}, \pi^{\prime \prime}\right)$ be algebraic Lefschetz fibrations such that $\left.\pi^{\prime \prime}\right|_{E^{\prime \prime}}=\pi^{\prime}$. Let $\theta^{\prime}$ (resp. $\theta^{\prime \prime}$ ) be a convex symplectic structure on $E^{\prime}$ (resp. $E^{\prime \prime}$ ) constructed as in Example 2.8 such that $d \theta^{\prime \prime}=\left.d \theta^{\prime}\right|_{E^{\prime \prime}}$. We assume that the real dimension of $E^{\prime}$ and $E^{\prime \prime}$ is 4 or higher.

Lemma 8.6 Suppose that all the singular points of $\pi^{\prime}$ are contained in $E^{\prime \prime}$. Then there exists a convex symplectic structure $\theta_{1}^{\prime}\left(\right.$ resp. $\left.\theta_{1}^{\prime \prime}\right)$ on $E^{\prime}$ (resp. $\left.E^{\prime \prime}\right)$ such that:

(1) $\left(E^{\prime}, \pi^{\prime}, \theta_{1}^{\prime}\right)\left(\operatorname{resp} .\left(E^{\prime \prime}, \pi^{\prime \prime}, \theta_{1}^{\prime \prime}\right)\right)$ are Lefschetz fibrations without boundary.

(2) $\left.d \theta_{1}^{\prime}\right|_{E^{\prime \prime}}=d \theta_{1}^{\prime \prime}$.

(3) All the parallel transport maps are trivial on a neighbourhood $N$ of $E^{\prime} \backslash E^{\prime \prime}$, and $E^{\prime} \backslash N$ is relatively compact when restricted to each fibre.

(4) For each smooth fibre $F^{\prime}$ of $\pi^{\prime},\left.\theta^{\prime}\right|_{F^{\prime}}=\left.\theta_{1}^{\prime}\right|_{F^{\prime}}+d R$ for some compactly supported function $R$. We have a similar statement for $\left(E^{\prime \prime}, \pi^{\prime \prime}\right)$.

(5) $\left(E^{\prime}, \theta_{1}^{\prime}\right)\left(\right.$ resp. $\left.\left(E^{\prime \prime}, \theta_{1}^{\prime \prime}\right)\right)$ is convex symplectic deformation equivalent to $\left(E^{\prime}, \theta^{\prime}\right)$ (resp. $\left.\left(E^{\prime \prime}, \theta^{\prime \prime}\right)\right)$.

Proof We divide this proof into 3 steps. In the first step we construct the Lefschetz fibration without boundary $\left(E^{\prime}, \pi^{\prime}, \theta_{1}^{\prime}\right)$. In the second step we construct $\left(E^{\prime \prime}, \pi^{\prime \prime}, \theta_{1}^{\prime \prime}\right)$. In the third step we show that $\left(E^{\prime}, \theta_{1}^{\prime}\right)\left(\right.$ resp. $\left.\left(E^{\prime \prime}, \theta_{1}^{\prime \prime}\right)\right)$ is convex deformation equivalent to $\left(E^{\prime}, \theta^{\prime}\right)$ (resp. $\left.\left(E^{\prime \prime}, \theta^{\prime \prime}\right)\right)$.

Step 1 We will use ideas from [26, Section 19b]. The map $\pi^{\prime}$ has well defined parallel transport maps by Theorem 8.5. We have the same for $\left(E^{\prime \prime}, \pi^{\prime \prime}\right)$. Suppose without loss of generality that $0 \in \mathbb{C}$ is a regular point of these fibrations. Let $Q^{\prime}:=\pi^{\prime-1}(0)$, $Q^{\prime \prime}:=Q \cap E^{\prime \prime}$. Consider the family of radial lines in $\mathbb{C}$ coming out of 0 . Let $L$ be one of these radial lines which passes through a critical value $l$ of $\pi^{\prime}$. We can write $L=L_{1} \cup L_{2}$ where $L_{1}$ is the line joining 0 and $l$, and $L_{1} \cap L_{2}=\{l\}$. We now 
Radial lines coming from the origin

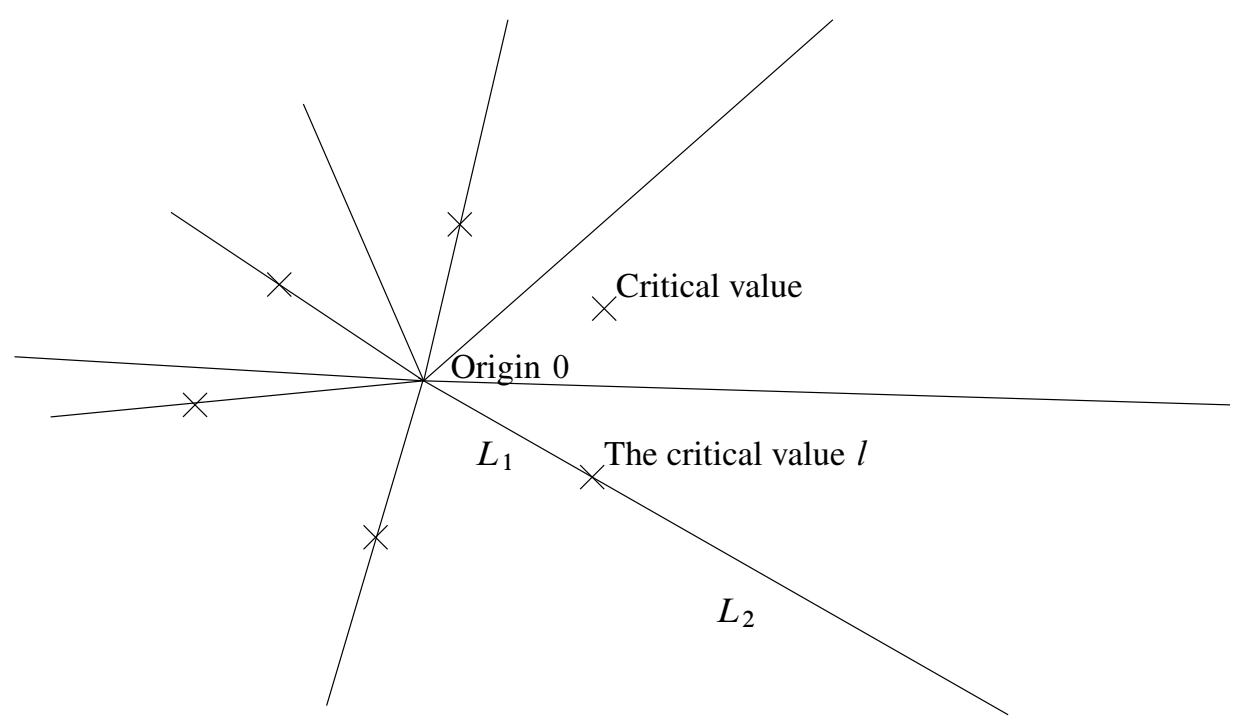

have vanishing thimbles $V_{1}$ and $V_{2}$ of $l$ covering $L_{1}$ and $L_{2}$. A vanishing thimble covering a line $L_{i}$ is just the set of points in $\pi^{\prime-1}\left(L_{i}\right)$ which parallel transport along $L_{i}$ into the critical point associated to the critical value $l$. Let $V$ be the union of all such thimbles for all radial lines passing through critical values of $\pi^{\prime}$. We can use this to construct a map $\rho: E^{\prime} \backslash V \rightarrow \mathbb{C} \times Q^{\prime}$. The map is constructed as follows: Let $x$ be a point in $E^{\prime} \backslash V$. Then we can parallel transport $x$ along a radial line to a point $a$ in $Q^{\prime}$. Then $\rho(x):=\left(\pi^{\prime}(x), a\right)$. Let $X=V \cap Q^{\prime}$ and $W:=Q^{\prime} \backslash X$. Then $\left.\rho\right|_{E^{\prime} \backslash V}: E^{\prime} \backslash V \rightarrow \mathbb{C} \times W$ is a diffeomorphism. Let $\varpi:=\left(\left.\rho\right|_{E^{\prime} \backslash V}\right)^{-1}$. Let $\theta_{Q^{\prime}}:=\left.\theta^{\prime}\right|_{Q^{\prime}}$. From now on, if we have a differential form $q$ on $\mathbb{C} \times W$, then we will just write $q$ instead of $\rho^{*} q$ to clean up notation.

Because parallel transport maps are exact, we have: $\left.\theta^{\prime}\right|_{E^{\prime} \backslash V}=\theta_{Q^{\prime}}+\kappa^{\prime}+d R^{\prime}$ where $\kappa^{\prime}$ is a 1 -form satisfying $i^{*} \kappa^{\prime}=0$ for all maps $i$ where $i$ is the inclusion map of any fibre of $\pi^{\prime}$ into $E^{\prime}$, and $R$ is some function on $\mathbb{C} \times W$. Let $\bar{f}: W \rightarrow \mathbb{R}$ be a function which is equal to 1 near $X$ and is 0 outside some relatively compact neighbourhood of $X$. We extend $\bar{f}$ by parallel transport along these radial lines to a map $g: E^{\prime} \backslash V \rightarrow \mathbb{R}$. Then we extend $g$ to a map $f: E^{\prime} \rightarrow \mathbb{R}$ as $g$ is constant near $V$. We will also assume that $g$ is only nonzero inside $E^{\prime \prime}$ because parallel transport maps are well defined for $\left(E^{\prime \prime}, \pi^{\prime \prime}, \theta^{\prime \prime}\right)$, hence $V \subset E^{\prime \prime}$. We define

$$
\theta_{f}^{\prime}:=\theta_{Q^{\prime}}+g \kappa^{\prime}+d\left(g R^{\prime}\right)
$$


This form extends over $V$ because $\theta_{f}^{\prime}=\theta^{\prime}$ near $V$ (where $g=1$ ). The 1 -form $\theta_{f}^{\prime}$ makes $\pi^{\prime}$ into a Lefschetz fibration without boundary where each of the fibres have a convex symplectic structure. We define $\theta_{1}^{\prime}:=\theta_{f}^{\prime}$.

Step 2 Let $Q^{\prime \prime}:=\pi^{\prime \prime-1}(0) \subset E^{\prime \prime}$. We also have that

$$
\theta^{\prime \prime}=\theta_{Q^{\prime \prime}}+\kappa^{\prime \prime}+d R^{\prime \prime} .
$$

Here, $\kappa^{\prime \prime}$ is a $1-$ form on $E^{\prime \prime}$ satisfying $i^{*} \kappa^{\prime \prime}=0$ for all maps $i$ where $i$ is the inclusion map of a fibre of $\pi^{\prime \prime}$ into $E^{\prime \prime}$, and $R^{\prime \prime}$ is some function on $E^{\prime \prime} \cap(\mathbb{C} \times W)$. Because $d \theta^{\prime \prime}=d \theta^{\prime}$, we have that $d \kappa^{\prime}=d \kappa^{\prime \prime}$. This means that $\beta:=\kappa^{\prime}-\kappa^{\prime \prime}$ is a closed 1 -form in $E^{\prime \prime}$. We can also show that $\beta$ is exact as follows: Let $l: \mathbb{S}^{1} \rightarrow E^{\prime \prime}$ be a loop. Because we are in dimension 4 or higher, we can perturb the loop so that it doesn't intersect the radial vanishing thimbles described above. We can then deform $l$ using parallel transport to a loop $l^{\prime}$ contained in a smooth fibre $F$. We have $\left.\beta\right|_{F}=0$ which means that $\int_{l} \beta=\int_{l^{\prime}} \beta=0$. Hence $\beta=d L$ for some $L: E^{\prime \prime} \rightarrow \mathbb{R}$. We define

$$
\theta_{f}^{\prime \prime}:=\theta_{Q^{\prime \prime}}+g \kappa^{\prime}+d(g L)+d\left(g R^{\prime}\right) .
$$

We have $d \theta_{f}^{\prime \prime}=d \theta_{f}^{\prime}$, hence this makes $\left(E^{\prime \prime}, \pi^{\prime \prime}\right)$ into a well defined symplectic subfibration of $E^{\prime}$. We define $\theta_{1}^{\prime \prime}:=\theta_{f}^{\prime \prime}$.

Step 3 We can deform $\bar{f}$ through functions which are trivial at infinity to some $\bar{f}^{\prime}$ where $\bar{f}^{\prime}=0$ outside some large compact set, and $\left(\bar{f}^{\prime}\right)^{-1}(1)$ contains an arbitrarily large compact set $K \subset F$. We can construct $f^{\prime}: E^{\prime} \rightarrow \mathbb{R}$ using $\bar{f}^{\prime}$ in the same way that we constructed $f$ from $\bar{f}$ and the deformation from $\bar{f}$ to $\bar{f}^{\prime}$ induces a deformation from $f$ to $f^{\prime}$. We can choose a convex symplectic structure $\theta_{S}$ on the base so that $\left(E^{\prime}, \theta_{f}^{\prime}+\pi^{\prime *} \theta_{S}\right)$ and $\left(E^{\prime}, \theta_{f^{\prime}}^{\prime}+\pi^{\prime *} \theta_{S}\right)$ are convex symplectic manifolds. Hence $\theta_{f}^{\prime}+\pi^{\prime *} \theta_{S}$ is convex deformation equivalent to $\theta_{f^{\prime}}^{\prime}+\pi^{\prime *} \theta_{S}$. If we choose $K$ large enough we get that $\theta_{f^{\prime}}^{\prime}+\pi^{\prime *} \theta_{S}$ is convex deformation equivalent to $\left(E^{\prime}, \theta^{\prime}+\pi^{\prime *} \theta_{S}\right)$ by Lemma 8.2 and Lemma 8.3, and hence is convex deformation equivalent to $\left(E^{\prime}, \theta^{\prime}\right)$.

Because $\theta_{f}^{\prime \prime}$ is described in a very similar way to $\theta_{f}^{\prime}$, we can use the same argument as above to show that $\left(E^{\prime \prime}, \theta^{\prime \prime}\right)$ is convex deformation equivalent to $\left(E^{\prime \prime}, \theta_{f}^{\prime \prime}+\pi^{*} \theta_{S, 1}\right)$. The 1 -form $\theta_{S, 1}$ is a convex symplectic structure on the base making $\theta_{f}^{\prime \prime}+\pi^{*} \theta_{S, 1}$ into a convex symplectic structure.

Let $X, D, M$ be as in Theorem 2.32. This means that $Z$ is an irreducible divisor in $X$ and $q \in(Z \cap M)$ is a point in the smooth part of $Z$. There is a rational function $m$ on $X$ which is holomorphic on $M$ such that $\overline{m^{-1}(0)}$ is reduced and irreducible and $Z=\overline{m^{-1}(0)}$. We have $M^{\prime}:=\operatorname{Kalmod}(M,(Z \cap M),\{q\})$, and $M^{\prime \prime}:=M \backslash Z$. We also have $\operatorname{dim}_{\mathbb{C}} X \geq 3$. 
Lemma 8.7 There exist algebraic Lefschetz fibrations

$$
p^{\prime}: M^{\prime} \rightarrow \mathbb{C}, \quad p^{\prime \prime}: M^{\prime \prime} \rightarrow \mathbb{C}
$$

such that $p^{\prime \prime}$ is a subfibration of $p^{\prime}$ (ie $p^{\prime} \circ$ (inclusion) $\left.=p^{\prime \prime}\right)$. Also, if $F^{\prime}\left(\right.$ resp. $\left.F^{\prime \prime}\right)$ is a page of $p^{\prime}$ (resp. $\left.p^{\prime \prime}\right)$, then $F^{\prime}$ is the proper transform of $F^{\prime \prime}$ in $\mathrm{Bl}_{q} X$. The singularities of $p^{\prime}$ are contained in $M^{\prime \prime}$.

Proof Let $Q$ be an effective ample line bundle on $X$ with support equal to $D$ and such that $Q^{\prime \prime}:=\overline{m^{-1}(0)}+Q$ is ample. Let $s^{\prime \prime}, t^{\prime \prime}$ be sections of $Q^{\prime \prime}$ such that $s^{\prime \prime-1}(0)=\overline{m^{-1}(0)}+Q$. We choose $t^{\prime \prime}$ such that

$$
p^{\prime \prime}=\frac{t^{\prime \prime}}{s^{\prime \prime}}: M^{\prime \prime} \rightarrow \mathbb{C}
$$

is some algebraic Lefschetz fibration on $M^{\prime \prime}$. Let $\bar{F}^{\prime \prime}$ be the closure of one of the smooth fibres of $p^{\prime \prime}$ in $M$. We can move the point $p$ to somewhere in the smooth part of $\bar{F}^{\prime \prime} \cap Z$ as the smooth part of $Z$ is connected (as $Z$ is irreducible); $M^{\prime}$ is unchanged up to Stein deformation. NB here we use $\operatorname{dim}_{\mathbb{C}} X \geq 2$.

Remember $b$ is the blowdown map $b: \mathrm{Bl}_{q} X \rightarrow X$. Let $s^{\prime}:=b^{*} s^{\prime \prime}$ and $t^{\prime}:=b^{*} t^{\prime \prime}$. Let $\Delta$ be the exceptional divisor $b^{-1}(p)$. The divisor $s^{\prime-1}(0)$ is equal to $\Delta+$ other divisors. We can choose an effective divisor $K^{\prime}$ with support equal to the boundary divisor $D^{\prime}$ of $M^{\prime}$ in $\mathrm{Bl}_{q} X$ such that $K^{\prime \prime}:=K^{\prime}-\Delta$ is ample. Hence, we can choose a meromorphic section $h$ of $K^{\prime \prime}$ whose zero set is contained in $D^{\prime}$, and such that $h$ has a pole of order 1 along the exceptional divisor and such that $h$ is holomorphic away from $D^{\prime} \cup \Delta$. Let $L$ be the line bundle associated to $K^{\prime \prime}$. This means that $s^{\prime} \otimes h \in H^{0}\left(\Im\left(L \otimes b^{*} Q^{\prime \prime}\right)\right)$ is nonzero away from $D^{\prime}$. We let $p^{\prime}:=\left(t^{\prime} \otimes h\right) /\left(s^{\prime} \otimes h\right)$. This means that $\left.p^{\prime}\right|_{M^{\prime \prime}}=p^{\prime \prime}$. Because $q$ is in the smooth locus of $\bar{F}^{\prime \prime} \cap Z$ and $\bar{F}^{\prime \prime}$ is transverse to $Z$, we have that the closure of any smooth fibre of $p^{\prime}$ intersects each stratum of $D^{\prime}$ transversally.

We can choose holomorphic coordinates $z_{1} \cdots z_{n}$ on an open set $U$ of $p$ and a holomorphic trivialisation of $Q^{\prime \prime}$ such that $s^{\prime \prime}=z_{1}$ and $t^{\prime \prime}=z_{2}$. We then blow up at the point $p$. Locally around $p$, we have a subvariety of $U \times \mathbb{P}^{n}$ defined by $Z_{i} z_{j}=Z_{j} z_{i}$ where $Z_{1} \cdots Z_{n}$ are projective coordinates for $\mathbb{P}^{n}$. We choose the chart $Z_{1}=1$. This has local holomorphic coordinates $z_{1}, Z_{2}, Z_{3}, \ldots, Z_{n}$. We can choose a trivialisation of $K^{\prime \prime}$ so that the section $h$ is equal to $1 / z_{1}$. This means that locally $b^{*} s^{\prime \prime}=Z_{1}$ and $b^{*} t^{\prime \prime}=Z_{2} z_{1}$. Hence locally, $s^{\prime}=1$ and $t^{\prime}=Z_{2}$ which means that $p^{\prime}=Z_{2}$. This means that $p^{\prime}$ has no singular points near $\Delta$. Hence $p^{\prime}$ is also an algebraic Lefschetz fibration which coincides with $p^{\prime \prime}$ away from $\Delta$ and such all the singular points of $p^{\prime}$ are the same as the singular points of $p^{\prime \prime}$. 
Lemma 8.8 Let $F^{\prime}$ (resp. $\left.F^{\prime \prime}\right)$ be a fibre of $p^{\prime}$ (resp. $\left.p^{\prime \prime}\right)$. Let $K$ be a compact set in $F^{\prime \prime}$. There is a Stein structure $J$ on $F^{\prime}$ (depending on $K$ ) such that any $J-$ holomorphic $u: T \rightarrow F^{\prime}$, where $T$ is a compact Riemann surface with boundary, has the property that $u(T) \subset F^{\prime \prime}$ if $u(\partial T) \subset F^{\prime \prime}$.

Proof Let $G$ be the closure of $F^{\prime \prime}$ in $M$. Then $F^{\prime}$ is biholomorphic to the complex $\operatorname{manifold} \operatorname{Kalmod}(G, G \cap Z,\{q\})$. Let $\phi_{G}$ be a Stein function for $G$. The compact set $K$ is contained in $\phi_{G}^{-1}(C)$ for some large $C$. Let $q^{\prime}$ be a point outside $\phi_{G}^{-1}(C)$ which is contained in the smooth part of $G \cap Z$. Because $\operatorname{dim}_{\mathbb{C}} G \cap Z \geq 2$, we can assume that $q$ and $q^{\prime}$ are in the same irreducible component $U$ of $G \cap Z$. This is where we use the assumption that $\operatorname{dim}_{\mathbb{C}} X \geq 3$. The manifold $G^{\prime}:=\operatorname{Kalmod}\left(G, G \cap Z,\left\{q^{\prime}\right\}\right)$ is naturally a Stein manifold by Example 2.8. By the comment after Lemma 8.1, we have that $G^{\prime}$ is Stein deformation equivalent to $F^{\prime}$ such that it also induces a Stein deformation on $M^{\prime \prime}$. The Stein deformation is induced from moving $q^{\prime}$ smoothly down to $q$ inside the smooth part of $U \subset G \cap Z$ (Note: $U$ is irreducible, hence the smooth part of $U$ is connected). This induces a Stein deformation of $M^{\prime}$ and $M^{\prime \prime}$ which in turn induces a Stein deformation of $F^{\prime}$ and $F^{\prime \prime}$.

From now on we assume that the symplectic structures on $F^{\prime}$ and $G^{\prime}$ are complete by [27, Lemma 6]. We can also ensure that the above Stein deformation between $F^{\prime}$ and $G^{\prime}$ is complete and finite type by the same lemma, hence by [27, Lemma 5] we have a symplectomorphism $h: F^{\prime} \rightarrow G^{\prime}$ induced by this Stein deformation. Let $J_{G^{\prime}}$ be the natural complex structure on $G^{\prime}$. Let $J:=h^{*} J_{G^{\prime}}$. Then if $T$ is a $J$-holomorphic curve in $F^{\prime}$ with boundary inside $K$ then $h(T)$ is a holomorphic curve in $G^{\prime}$ with boundary in $h(K)$. We can blow down this curve to give a holomorphic curve $T^{\prime}$ in $G$ with boundary in $b(h(K))$. We can ensure that $b(h(K))$ is contained in $\phi_{G}^{-1}(C)$. If $T$ passes through the blowup of $q$, then $T^{\prime}$ passes through $q^{\prime}$. This means that $\phi_{G} \circ T^{\prime}$ has an interior maximum outside $\phi_{G}^{-1}(C)$, but this is impossible. Hence the curve $T$ must be contained in $F^{\prime \prime}$.

We can now apply the above lemmas to prove Theorem 2.31. We can apply Lemma 8.6 to $p^{\prime}$ and $p^{\prime \prime}$ to get symplectic fibrations $\left(M^{\prime}, p^{\prime}, \theta_{1}^{\prime}\right)$ and $\left(M^{\prime \prime}, p^{\prime \prime}, \theta_{1}^{\prime \prime}\right)$. These fibrations are Lefschetz without boundary. We can cut down the fibres to Stein domains $\overline{F^{\prime}}$ and $\overline{F^{\prime \prime}}$ where $\overline{F^{\prime \prime}}, \overline{F^{\prime}}$ are large enough so that the support of all the monodromy maps of $\left(M^{\prime}, p^{\prime}, \theta_{1}^{\prime}\right)$ are contained in $\overline{F^{\prime \prime}}$ and $\overline{F^{\prime \prime}} \subset \overline{F^{\prime}}$. We can also remove the cylindrical end from the base. This will make $p^{\prime}$ and $p^{\prime \prime}$ into Lefschetz fibrations $\left(E^{\prime}, \pi^{\prime}\right)$ and $\left(E^{\prime \prime}, \pi^{\prime \prime}\right)$ respectively. Note that if we have a holomorphic curve $T$ in $\overline{F^{\prime}}$ with boundary in $\overline{F^{\prime \prime}}$, Lemma 8.8 implies that it is contained in $F^{\prime \prime} \cap \overline{F^{\prime}}$. The Stein maximum principle [20, Lemma 1.5] ensures that $T$ is contained in $\overline{F^{\prime \prime}}$. Hence we get that Theorem 2.31 is a consequence of Lemma 8.6 and Lemma 8.8. 


\section{Appendix B: Stein structures and cylindrical ends}

The problem with Stein structures is that the complex structure associated with them does not behave well with respect to cylindrical ends. Cylindrical ends here means that near infinity, the convex symplectic manifold is exact symplectomorphic to $(\Delta \times[1, \infty), r \alpha)$ where $r \in[1, \infty)$ and $\alpha$ is a contact form on $\Delta$. The almost complex structure is convex with respect to this cylindrical end if $d r \circ J=-\alpha$. We will deal with this problem in this section.

Let $(M, J, \phi)$ be a complete finite-type Stein manifold with $\theta=-d^{c} \phi$ and $\omega=d \theta$. Let $c \gg 0$ be greater than the highest critical value of $\phi$.

Theorem 9.1 There exists a complete finite type convex symplectic structure $\left(M, \theta_{1}\right)$ with the following properties:

(1) It has a cylindrical end with an almost complex structure $J_{1}$ which is convex at infinity.

(2) $J_{1}=J$ and $\theta_{1}=\theta$ in the region $\{\phi \leq c\}$.

(3) Any $J_{1}$ holomorphic curve with boundary in $\{\phi=c\}$ is contained in $\{\phi \leq c\}$.

(4) It is convex deformation equivalent to $(M, \theta)$ via a convex deformation $\left(M, \theta_{t}\right)$ where $\left.\theta_{t}\right|_{\{\phi \leq c\}}=\left.\theta\right|_{\{\phi \leq c\}}$ for $t \in[0,1]$.

Proof Let $\lambda:=\nabla \phi$ and $\Delta:=\phi^{-1}(c+1)$. We define $G: M \rightarrow \mathbb{R}, G=1 /\|\nabla \phi\|^{2}$ where $\|\cdot\|$ is the norm defined using the metric $\omega(\cdot, J \cdot)$. Let $\lambda^{\prime}:=G \lambda$. Let $F_{t}: M \rightarrow M$ be the flow of $\lambda^{\prime}$. This exists for all time because $\phi$ is unbounded and $\mathcal{L}_{\lambda^{\prime}} \phi=1$ which implies that $\phi$ increases linearly with $t(\mathcal{L}$ here means Lie derivative). We have an embedding $\Phi: \Delta \times[1, \infty) \rightarrow M$ defined by $\Phi(a, r)=F_{\log r}(a)$ where $a \in \Delta \subset M$ and $r \in[1, \infty)$. Also, $\mathcal{L}_{\lambda^{\prime}} \theta=G \theta$. Hence, $\Phi^{*}(\theta)=f \alpha$ where $f: \Delta \times[1, \infty) \rightarrow \mathbb{R}, f(a, r):=1+\int_{0}^{r}(G \circ \Phi)(a, t) d t$ and $\alpha$ is the contact form $\left.\theta\right|_{\Delta}$ on $\Delta$.

We will now deform the 1 -form $f \alpha$ to a 1 -form $f^{\prime} \alpha$ such that $f^{\prime}=f$ near $r=1$ and $f^{\prime}=r$ near infinity. We define $\theta_{1}$ to be equal to $f^{\prime} \alpha$ in this cylindrical end and equal to $\theta$ away from this end. This means that for $r$ large, we have a cylindrical end with 1 -form $f^{\prime} \alpha=r \alpha$. If we have a function $g: \Delta \times[1, \infty) \rightarrow \mathbb{R}$, then $d(g \alpha)$ is nondegenerate if and only if $\partial g / \partial r>0$. Also, the Liouville vector field associated to $g \alpha$ is $(g /(\partial g / \partial r))(\partial / \partial r)$, and hence we have that this Liouville vector field is transverse to every level set $\{r=$ const $\}$ and pointing outwards. If $(g /(\partial g / \partial r))$ is bounded above by any polynomial, then the respective Liouville vector field is complete. We define $f^{\prime}: \Delta \times[1, \infty) \rightarrow \mathbb{R}$ such that $f^{\prime}=f$ near $r=1, f^{\prime}=r$ near infinity 
and $\partial f^{\prime} / \partial r>0$. This gives a complete finite type convex symplectic structure $\theta_{1}$ on $M$ as we can extend $f^{\prime} \alpha$ outside $M$ as $f^{\prime}=f$ near $r=1$. We can join $f$ to $f^{\prime}$ via a smooth family of functions with $f_{t}(t \in[0,1])$ where $\partial f_{t} / \partial r>0$ and such that $f_{t}=f$ near $r=1$. This gives us a convex deformation from $\theta$ to $\theta^{\prime}$.

We now need to construct our almost complex structure $J_{1}$. We have $\mathcal{L}_{\lambda^{\prime}} \phi=$ $G d \phi(\nabla \phi)=1$. This means that $\Phi^{*}(\phi)=\log r$ so the level sets of $\phi$ coincide with the level sets of $\log r$. By abuse of notation we will just write $J$ for the pullback $\Phi^{*} J$ and we will write $\phi$ for $\log r$. We have two orthogonal symplectic vector subbundles of the tangent bundle $\Phi^{*}(T M)=T(\Delta \times[1, \infty))$ whose direct sum is the entire tangent bundle (the symplectic structure we are dealing with here is $\theta_{1}$ ). These are: $V_{1}:=\operatorname{Ker}\left(\theta_{1}\right) \cap \operatorname{Ker}(d r)$ and $V_{2}:=\operatorname{Span}\left(\partial / \partial r, X_{r}\right)$ where $X_{r}$ is the Hamiltonian flow of $r$. The problem is that $J$ is not necessarily compatible with $d \theta_{1}$, so we need to deform it so that it is. However, near $r=1, J$ is in fact compatible with $d \theta_{1}$ because $\theta=\theta_{1}$ in some region $\Xi:=\{r \leq 1+\epsilon\}$. Inside $\Xi$, we have that: $\left.J\right|_{V_{1}}$ and $\left.J\right|_{V_{2}}$ are holomorphic subbundles of $\Phi^{*}(T M)$. There exists a complex structure $J_{V_{1}}$ (resp. $J_{V_{2}}$ ) on the vector bundle $V_{1}$ (resp. $V_{2}$ ) compatible with $\left.d \theta_{1}\right|_{V_{1}}$ (resp. $\left.d \theta_{1}\right|_{V_{2}}$ ) such that, $J_{V_{1}}=\left.J\right|_{V_{1}}$ (resp. $J_{V_{2}}=\left.J\right|_{V_{2}}$ ) when restricted to $\Xi$. Because $V_{1} \oplus V_{2}=\Phi^{*}(T M)$, this gives us an almost complex structure $J_{1}$ on $\Phi^{*}(T M)$ compatible with $d \theta_{1}$ which is equal to $J$ in the region $\Xi$. We can choose $J_{V_{1}}$ and $J_{V_{2}}$ so that $J_{V_{2}}(\partial / \partial r)=-(1 / r) X_{r}$ for $r \gg 0$ and $J_{V_{1}}$ is invariant under the flow of $\partial / \partial r$ for $r \gg 0$. This ensures that $J_{1}$ is convex at infinity. Also, we have that $r$ is plurisubharmonic with respect to $J_{1}$ hence any $J_{1}$ holomorphic curve with boundary in $\{r=1\}$ is contained in $\{r \leq 1\}$. Hence property (3) is satisfied.

\section{Appendix C: Transfer maps and handle attaching}

The purpose of this section is to show that symplectic homology is additive as a ring under end connect sums. This was already done by Cieliebak [7] but without taking into account the ring structure. Throughout this section, let $(M, \theta),\left(M^{\prime}, \theta^{\prime}\right)$ be compact convex symplectic manifolds such that $M^{\prime}$ is an exact submanifold of $M$ of codimension 0 . We let $C:=N \times[1, \infty)$ be a cylindrical end of $M$ where $\theta=r \alpha$, $\alpha$ is a contact form on $N$, and $r$ is the coordinate for $[1, \infty)$. Similarly we have a cylindrical end $C^{\prime}$ of $M^{\prime}$. Let $H: M \rightarrow \mathbb{R}$ be an admissible Hamiltonian with an almost complex structure $J$, convex at infinity. Let $\mathrm{SH}_{*}^{(-\infty, a)}(M, H, J)$ be the group generated by orbits of action $<a$. For $b \geq a$, we define

$$
\mathrm{SH}_{*}^{[a, b)}(M, H, J):=\mathrm{SH}_{*}^{(-\infty, b)}(M, H, J) / \mathrm{SH}_{*}^{(-\infty, a)}(M, H, J) .
$$




\subsection{Weak cofinal families}

Definition 10.1 We say that the pair $(H, J)$ is weakly admissible if there exists an $f: N \rightarrow \mathbb{R}$ and a constant $b$ such that for $r \gg 0, H=r e^{-f}+b$ and $d\left(r e^{-f}\right) \circ J=-\theta$.

Every admissible pair $(H, J)$ is weakly admissible with $f=$ const. Symplectic homology $\mathrm{SH}_{*}(M)$ is defined as a direct limit of $\mathrm{SH}_{*}(M, H, J)$ with respect to admissible pairs ordered by $\leq$. We wish to replace "admissible" with "weakly admissible". The reason why we wish to do this is because in Section 10.3 we carefully construct a cofinal family of weakly admissible pairs to show that symplectic homology behaves well under end connect sums. We construct a partial order $\leq$ on weakly admissible pairs as follows: $\left(H_{0}, J_{0}\right) \leq\left(H_{1}, J_{1}\right)$ if and only if $H_{0} \leq H_{1}$. We will show that

$$
\mathrm{SH}_{*}(M):=\underset{(\overrightarrow{H, J})}{\lim _{\overrightarrow{1}}} \mathrm{SH}_{*}(M, H, J)
$$

where the direct limit is taken over weakly admissible pairs $(H, J)$ ordered by $\leq$. Note that a family of weakly admissible Hamiltonians $\left(H_{S}, J_{S}\right)$ is cofinal with respect to $\leq$ if the corresponding functions $f_{s}: N \rightarrow \mathbb{R}$ tend uniformly to $-\infty$ as $s$ tends to $\infty$. In order to ensure that this direct limit exists, we will show that if $\left(H_{0}, J_{0}\right) \leq\left(H_{1}, J_{1}\right)$, then there is a natural map of rings $\mathrm{SH}_{*}\left(M, H_{0}, J_{0}\right) \rightarrow \mathrm{SH}_{*}\left(M, H_{1}, J_{1}\right)$.

This map will be a continuation map. In order for a continuation map to be well defined, we need a family of Hamiltonians $T_{s}$ joining $H_{0}$ and $H_{1}$ such that solutions of the parameterized Floer equation $\partial_{s} u+J_{t} \partial_{t} u=\nabla^{g_{t}} T_{s}$ joining orbits of $H_{0}$ and $H_{1}$ stay inside some compact set. To ensure this, we flatten $H_{i}$ so that it is constant outside some large compact set, and so that all the additional orbits created have very negative action. We do this as follows:

Let $D$ be a constant such that any orbit of $H_{0}$ or $H_{1}$ has action greater than $D$. Then $\mathrm{SH}_{*}\left(M, H_{i}, J_{i}\right) \cong \mathrm{SH}_{*}^{[D, \infty)}\left(M, H_{i}, J_{i}\right)$. Near infinity, we have that $H_{i}=R_{i}+b_{i}$ where $R_{i}=r e^{-f_{i}}$. We wish to create a new Hamiltonian $K_{i}$ such that $K_{i}=H_{i}$ on $R_{i} \leq B$ where $B \gg 0$, and such that $K_{i}$ is constant in $\left\{R_{i}>B+1\right\}$ where all the additional orbits have action less than $D$. We assume that all the orbits of $H_{0}$ and $H_{1}$ lie in a compact set. We have a cylindrical end $C_{i}:=N_{i} \times[K, \infty)$ where $N_{i}$ is the contact manifold $\left\{r e^{-f_{i}}=1\right\}$ with contact form $\left.\theta\right|_{N_{i}}$ and $R_{i}$ is the coordinate for $[K, \infty)$. So, $H_{i}$ is linear with slope 1 on this cylindrical end. Because all the orbits of $H_{i}$ lie in a compact set, there are no Reeb orbits of length 1 in the contact manifold $N_{i}$. Choose $\epsilon>0$ such that the length of any Reeb orbit of $N_{0}$ or $N_{1}$ is of distance more than $\epsilon$ from 1. We assume that $B$ is large enough so that $H_{i}$ is linear with respect to the cylindrical end $C_{i}$ in $R_{i} \geq B$ and such that $B \epsilon>-b_{i}-D$ for $i=0,1$. Finally we 
let $K_{i}$ be equal to $H_{i}$ in the region $\left\{R_{i} \leq B\right\}$, and $K_{i}=k_{i}\left(R_{i}\right)$ where $k_{i}$ is constant for $R_{i} \geq B+1$, and $k_{i}=R_{i}+b_{i}$ near $B$ and $k_{i}^{\prime} \leq 1$. This means that the orbits of $K_{i}$ in $\left\{R_{i} \leq B\right\}$ are the same as the orbits of $H_{i}$, and the orbits of $K_{i}$ in $\left\{R_{i}>B\right\}$ have action

$$
R_{i} k_{i}^{\prime}-k_{i}<R_{i}(1-\epsilon)-R_{i}-b_{i}<-B \epsilon-b_{i}<D .
$$

Hence all the orbits of $K_{i}$ of action greater than $D$ are the same as the orbits of $H_{i}$.

We now wish to create an almost complex structure $J_{i}^{0}$ as follows: we let $J_{i}^{0}=J_{i}$ for $R_{i} \leq B$ and for $R_{i} \geq B+1$, we let $J_{i}^{0}$ be convex with respect to the cylindrical end $C$ (ie $d r \circ J=-\theta$ ). Let $u$ be a cylinder or pair of pants satisfying Floer's equation [25, Formula 8.1] with respect to $\left(K_{i}, J_{i}^{0}\right)$ such that each cylindrical end of $u$ limits to a periodic orbit (or multiple of a periodic orbit in the pair of pants case) inside $\left\{R_{i} \leq B\right\}$. By [3, Lemma 7.2] we have that $u$ is contained in $\left\{R_{i} \leq B\right\}$. Note that we really perturb these Hamiltonians so that all the orbits are nondegenerate, and lie in a compact set. Lemma 7.2 from [3] still works in this case, as we can ensure the Hamiltonian stays the same in the region $B-1<R_{i}<B$. From now on if we deal with Hamiltonians which are constant at infinity, we are really perturbing them in such a way that this convexity argument from [3] still holds and such that $\mathrm{SH}_{*}$ is well defined for this Hamiltonian. Hence

$$
\mathrm{SH}_{*}\left(M, H_{i}, J_{i}\right) \cong \mathrm{SH}_{*}^{[D, \infty)}\left(M, H_{i}, J_{i}^{0}\right) \cong \mathrm{SH}_{*}^{[D, \infty)}\left(M, K_{i}, J_{i}^{0}\right) .
$$

We wish to create a continuation map

$$
\mathrm{SH}_{*}^{[D, \infty)}\left(M, K_{0}, J_{0}^{0}\right) \rightarrow \mathrm{SH}_{*}^{[D, \infty)}\left(M, K_{1}, J_{1}^{0}\right) .
$$

There exists a family of Hamiltonians $A_{s}$ connecting $K_{0}$ and $K_{1}$, and such that $A_{s}$ is monotonically increasing and $A_{s}$ is constant at infinity. We also join $J_{0}^{0}$ and $J_{1}^{0}$ with a family of almost complex structures which are convex with respect to the cylindrical end $C$ (ie $d r \circ J=-\theta$ ). If $f_{0}$ and $f_{1}$ are constant, then we have a monotone increasing family of admissible Hamiltonians $H_{s}^{1}$ joining $H_{0}$ and $H_{1}$, and almost complex structures $J_{s}$ joining $J_{0}$ and $J_{1}$. The standard continuation map

$$
\mathrm{SH}_{*}\left(M, H_{0}, J_{0}\right) \rightarrow \mathrm{SH}_{*}\left(M, H_{1}, J_{1}\right)
$$

involves counting solutions of a parameterized Floer equation with respect to $\left(H_{S}, J_{S}\right)$. We wish to show that this map is the same as the above continuation map from $K_{0}$ to $K_{1}$. In order to do this we construct an explicit family $\left(A_{S}, J_{S}\right)$ of Hamiltonians and almost complex structures so that the continuation map

$$
\mathrm{SH}_{*}^{[D, \infty)}\left(M, K_{0}, J_{0}^{0}\right) \rightarrow \mathrm{SH}_{*}^{[D, \infty)}\left(M, K_{1}, J_{1}^{0}\right)
$$


coincides with the standard continuation map

$$
\mathrm{SH}_{*}\left(M, H_{0}, J_{0}\right) \rightarrow \mathrm{SH}_{*}\left(M, H_{1}, J_{1}\right)
$$

under the isomorphism $\mathrm{SH}_{*}\left(M, H_{i}, J_{i}\right) \cong \mathrm{SH}_{*}^{[D, \infty)}\left(M, K_{i}, J_{i}^{0}\right)$. The Hamiltonians $H_{s}^{1}$ are of the form $h_{s}(r)$ for $r \geq P$ where $h_{s}^{\prime}$ is constant. We assume that the almost complex structures $J_{S}$ are convex with respect to the cylindrical end $C$ for $r \geq P$. Hence [3, Lemma 7.2] ensures all the Floer trajectories with respect to $\left(H_{S}, J_{S}\right)$ stay inside the compact set $r \leq P$. Also there is a constant $P^{\prime}$ such that $K_{i}$ is a function of $r$ for $r \geq P^{\prime} \geq P$. The definition of $K_{i}$ depends on a parameter $B$ which can be arbitrarily large. We can choose $B$ large enough so that $K_{i}=H_{i}$ in the region $r \leq P^{\prime}$. We choose the functions $A_{s}$ joining $K_{0}$ and $K_{1}$ so that $A_{s}$ is a function of $r$ for $r \geq P^{\prime}$. We can also assume that $J_{s}^{0}=J_{s}$. In order to show that the maps are the same, we need to show that any Floer trajectory associated to $\left(A_{s}, J_{s}^{0}\right)$ connecting orbits inside $\{r \leq P\}$ is contained in $\{r \leq P\}$. This follows from [3, Lemma 7.2].

\subsection{Transfer maps}

In this section we will construct a natural ring homomorphism: $\mathrm{SH}_{*}(M) \rightarrow \mathrm{SH}_{*}\left(M^{\prime}\right)$. We say that $H$ is called transfer admissible if $H \leq 0$ on $M^{\prime}$. We have: $\operatorname{SH}_{*}(M)=$ $\lim _{(H, J)} \mathrm{SH}_{*}(M, H, J)$ where the direct limit is taken over transfer admissible Hamiltonians ordered by $\leq$.

Lemma 10.2 We have an isomorphism of rings,

$$
\underset{(\overrightarrow{H, J})}{\lim } \mathrm{SH}_{*}^{[0, \infty)}(M, H, J) \cong \mathrm{SH}_{*}\left(M^{\prime}\right)
$$

where the direct limit is taken over transfer admissible Hamiltonians.

Proof We construct a particular cofinal family of transfer admissible Hamiltonians $H_{i}$ and show the above isomorphism of rings. We can embed $\widehat{M^{\prime}}$ into $\widehat{M}$ by Lemma 2.5. Our cylindrical end $C^{\prime}$ is then a subset of $M$. We assume that the action spectrum $\mathcal{S}:=\mathcal{S}\left(\partial M^{\prime}\right)$ is discrete and injective. Let $k: \mathbb{N} \rightarrow \mathbb{R} \backslash \mathcal{S}$ be a function such that $k(i)$ tends to infinity as $i$ tends to infinity. Let $\mu: \mathbb{N} \rightarrow \mathbb{R}$ be defined by $\operatorname{dist}(k(i), \mathcal{S})$ (dist $(a, B)$ is the shortest distance between $a$ and $B$ ). From now on we just write $k$ instead of $k(i)$, and similarly for $\mu$.

Define

$$
A=A(i):=6 k / \mu>k>1 .
$$

We can assume that $A>k>1$ because we can choose $k(i)$ to make $\mu(i)$ arbitrarily small whilst $k(i)$ is large. We also let $\epsilon:=\epsilon(i)$ tend to 0 as $i$ tends to infinity. We 
assume that $\left.H_{i}\right|_{M^{\prime}} \leq 0$, and has slope $k(i)$ on $1+\epsilon / k \leq r^{\prime} \leq A-\epsilon / k$. We also assume that on $1 \leq r^{\prime} \leq A, H_{i}=h\left(r^{\prime}\right)$ for some function $h$ where $h$ has nonnegative derivative $\leq k$. For $A \leq r^{\prime} \leq A+1$, we assume that $H_{i}$ is constant. Let $B$ be this constant. $B$ is arbitrarily close to $k(A-1)$. We can assume that $B \notin \mathcal{S}$. We now describe $H_{i}$ on the cylindrical end $C$. We keep $H_{i}$ constant until we reach $r=A+1+P$ where $P$ is some constant large enough so that $\left\{r^{\prime} \leq 1\right\} \subset\{r \leq P\}$. This means that $\left\{r^{\prime} \leq A+1\right\} \subset\{r \leq A+1+P\}$ as long as we embed $C^{\prime}$ in the same way as Lemma 2.5. We then let $H_{i}$ be of the form $f(r)$ for $r \geq A+1+P$ where $f^{\prime}<\frac{1}{2} k$ and has slope $\frac{1}{2} k$ for $r>A+1+P+\epsilon / k$.

Figure 4 shows a picture of what we have.

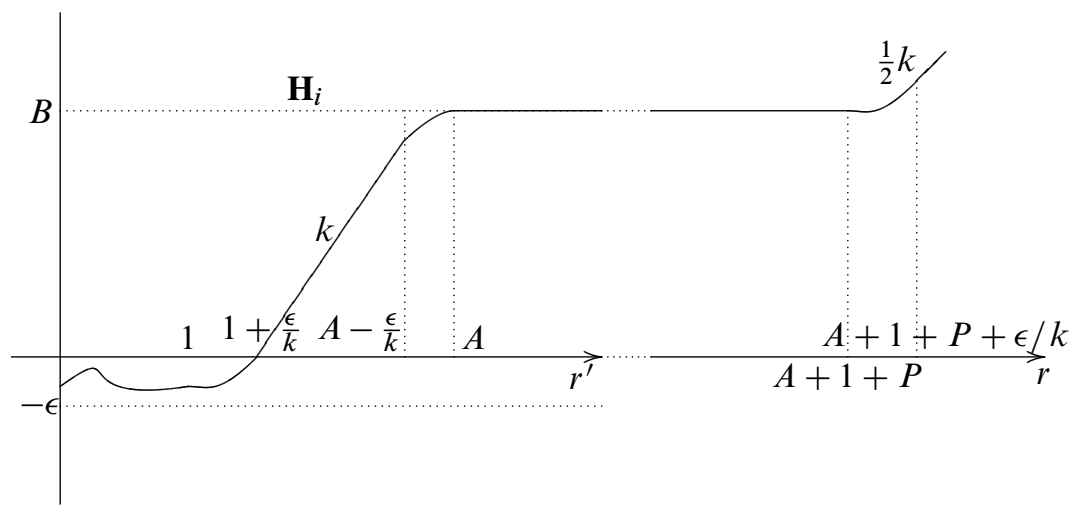

Figure 4

The action of an orbit on a level set $r^{\prime}=a$ is $h^{\prime}(a) a-h(a)$. The orbits near $r^{\prime}=1$ have positive action less than or equal to $k$. Let $p$ be a point on an orbit $o$ lying in the region $A-\epsilon / k \leq r^{\prime} \leq A$. The slope $h^{\prime}\left(r^{\prime}\right)$ of $H_{i}$ at $p$ is $\leq k-\mu$. Hence, the orbits near $r^{\prime}=A$ have action $\leq(k-\mu) A-B=-\mu A+k \rightarrow-\infty$ as $i \rightarrow \infty$. So, we can assume that these orbits have negative action. Also all the orbits in $\left\{r^{\prime} \geq A, r \leq A+1+P\right\}$ are fixed points, so have action $-B<0$. Finally, the orbits in $r>A+1+P$ have action: $\leq \frac{1}{2} k \cdot(A+1+P)-B=-\frac{1}{2} k A+\frac{3}{2} k+\frac{1}{2} P k \rightarrow-\infty$ as $i \rightarrow \infty$. Hence, we can assume that all the orbits of $H_{i}$ of nonnegative action lie in $r^{\prime}<1+\epsilon / k$.

We now need to show that any differential connecting two orbits of nonnegative action is contained entirely in $r^{\prime}<1+\epsilon / k$. By [3, Lemma 7.2], any differential connecting orbits of nonnegative action must be contained in $r^{\prime}<1+\epsilon / k$ as all the orbits of nonnegative action are contained in this region. A similar application of this lemma ensures that a pair of pants satisfying Floer type equations with similar Hamiltonians where the ends 
converge to orbits of nonnegative action must be contained in $r^{\prime}<1+\epsilon / k$. This means that we have maps $\mathrm{SH}_{*}^{[0, \infty)}\left(M, H_{i}, J\right) \cong \mathrm{SH}_{*}\left(M^{\prime}, H_{i}^{\prime}, J^{\prime}\right)$, where $H_{i}^{\prime}: \widehat{M^{\prime}} \rightarrow \mathbb{R}$ has slope $k$. Taking direct limits gives us a ring isomorphism

$$
\underset{(\overrightarrow{H, J})}{\lim } \mathrm{SH}_{*}^{[0, \infty)}(M, H, J) \cong \mathrm{SH}_{*}\left(M^{\prime}\right) \text {. }
$$

This lemma enables us to define a transfer map

$$
\mathrm{SH}_{*}(M) \cong \underset{(\overrightarrow{H, J})}{\lim _{\vec{H}}} \mathrm{SH}_{*}(M, H, J) \rightarrow \underset{(\overrightarrow{H, J})}{\lim _{*}} \mathrm{SH}_{*}^{[0, \infty)}(M, H, J) \cong \mathrm{SH}_{*}\left(M^{\prime}\right) .
$$

A Hamiltonian is called weakly transfer admissible if it is weakly admissible and is negative when restricted to $M^{\prime}$. We can combine the above results with the results of Section 10.1 to construct the above transfer map using a cofinal family of weakly transfer admissible Hamiltonians. We will need to construct a cofinal family of weakly transfer admissible Hamiltonians in Section 10.3 to show that a particular transfer map is an isomorphism of rings.

Here is an application of the transfer map:

Lemma 10.3 If $\mathrm{SH}_{*}(M)=0$, then $\mathrm{SH}_{*}\left(M^{\prime}\right)=0$.

Proof We have a commutative diagram:

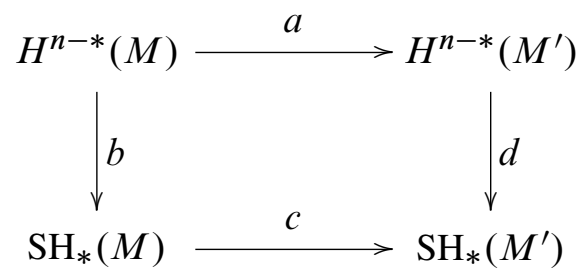

Suppose for a contradiction $\mathrm{SH}_{*}\left(M^{\prime}\right) \neq 0$. Then [25, Section 8] says that the map $d$ is nonzero in degree $n$. Also the map $a$ is an isomorphism in degree $n$. Hence $d \circ a$ is nonzero, and so $c \circ b=d \circ a$ is nonzero. This means that $\mathrm{SH}_{*}(M) \neq 0$ and we get a contradiction.

Corollary 10.4 If $M$ is subcritical and $\mathrm{SH}_{*}\left(M^{\prime}\right) \neq 0$, then $M^{\prime}$ cannot be embedded in $M$ as an exact codimension 0 submanifold. In particular, if $H_{1}\left(M^{\prime}\right)=0$ then $M^{\prime}$ cannot be symplectically embedded into $M$.

Proof By one of the applications of [21], we have that $\mathrm{SH}_{*}(M)=0$ because $M$ is subcritical. The result follows from the above lemma. 


\subsection{Handle attaching}

In this section we will prove Theorem 2.20. Recall its statement:

Theorem 2.20 Let $M, M^{\prime}$ be finite type Stein manifolds of real dimension greater than 2, then $\mathrm{SH}_{*}\left(M \#_{e} M^{\prime}\right) \cong \mathrm{SH}_{*}(M) \times \mathrm{SH}_{*}\left(M^{\prime}\right)$ as rings. Also the transfer map $\mathrm{SH}_{*}\left(M \#_{e} M^{\prime}\right) \rightarrow \mathrm{SH}_{*}(M)$ is just the natural projection

$$
\mathrm{SH}_{*}(M) \times \mathrm{SH}_{*}\left(M^{\prime}\right) \rightarrow \mathrm{SH}_{*}(M) .
$$

To prove this, we will show that attaching a symplectic 1 -handle to a compact convex symplectic manifold of dimension $\geq 2$ does not change symplectic homology. We will describe in more detail what it means to attach a symplectic 1-handle later. In fact we show that if $A$ is a compact convex symplectic manifold, and $A^{\prime}$ is equal to $A$ with a symplectic 1 -handle attached, then the natural transfer map $\mathrm{SH}_{*}\left(A^{\prime}\right) \rightarrow$ $\mathrm{SH}_{*}(A)$ is an isomorphism. This proves Theorem 2.20 for the following reason: The Stein manifold $M$ (resp. $M^{\prime}$ ) is convex deformation equivalent to $\widehat{N}$ (resp. $\widehat{N^{\prime}}$ ) where $N$ (resp. $N^{\prime}$ ) is a compact convex symplectic manifold. The end connect sum $M \#_{e} M^{\prime}$ is convex deformation equivalent to $\widehat{N^{\prime \prime}}$ where $N^{\prime \prime}$ is the disjoint union $N \sqcup N^{\prime}$ with a symplectic 1-handle joining each connected component. The symplectic homology of $N \sqcup N^{\prime}$ is the direct product $\mathrm{SH}_{*}(N) \times \mathrm{SH}_{*}\left(N^{\prime}\right)$. Also, the transfer map $\mathrm{SH}_{*}\left(N \sqcup N^{\prime}\right) \rightarrow \mathrm{SH}_{*}(N)$ is the natural projection $\mathrm{SH}_{*}(N) \times \mathrm{SH}_{*}\left(N^{\prime}\right) \rightarrow \mathrm{SH}_{*}(N)$. Hence assuming that adding a 1 -handle does not change symplectic homology, we have that $\mathrm{SH}_{*}\left(N^{\prime \prime}\right)$ is isomorphic to $\mathrm{SH}_{*}(N) \times \mathrm{SH}_{*}\left(N^{\prime}\right)$, hence $\mathrm{SH}_{*}\left(M \#_{e} M^{\prime}\right)$ is isomorphic to this product. This implies that $\mathrm{SH}_{*}\left(M \#_{e} M^{\prime}\right) \cong \mathrm{SH}_{*}(M) \times \mathrm{SH}_{*}\left(M^{\prime}\right)$ because $M$ (resp. $M^{\prime}$ ) is convex deformation equivalent to $N$ (resp. $N^{\prime}$ ). Also the natural transfer map from $\mathrm{SH}_{*}\left(M \#_{e} M^{\prime}\right)$ to $\mathrm{SH}_{*}(M)$ is the natural projection $\mathrm{SH}_{*}(M) \times \mathrm{SH}_{*}\left(M^{\prime}\right) \rightarrow \mathrm{SH}_{*}(M)$ because this corresponds to the composition of maps

$$
\mathrm{SH}_{*}\left(N^{\prime \prime}\right) \stackrel{\cong}{\rightarrow} \mathrm{SH}_{*}\left(N \sqcup N^{\prime}\right) \stackrel{\cong}{\rightarrow} \mathrm{SH}_{*}(N) \times \mathrm{SH}_{*}\left(N^{\prime}\right) \rightarrow \mathrm{SH}_{*}(N)
$$

We will now describe handle attaching in detail as in [7, Section 2.2]. The paper [12] or [8, Theorem 9.4] ensures that this construction corresponds to attaching a Stein 1-handle. We will define $\phi, p_{i}, q_{i}, X, \omega, \psi(x, y)$ as in [7, Section 2.2]. We will now remind the reader what these variables are: We set $k=1$, so we are describing 1 -handles only. We let $\mathbb{R}^{2 n}$ have coordinates $\left(p_{1}, q_{1}, \ldots, p_{n}, q_{n}\right)$.

$$
\omega:=\sum_{i} d p_{i} \wedge d q_{i}
$$




$$
\begin{aligned}
\phi & :=\frac{1}{4} \sum_{i=1}^{n-1}\left(q_{i}^{2}+p_{i}^{2}\right)+q_{n}^{2}-\frac{1}{2} p_{n}^{2} \\
X & :=\nabla \phi=\frac{1}{2} \sum_{i=1}^{n-1}\left(\frac{\partial}{\partial q_{i}}+\frac{\partial}{\partial p_{i}}\right)+2 \frac{\partial}{\partial q_{n}}-\frac{\partial}{\partial p_{n}}
\end{aligned}
$$

$\psi$ is a function of $x$ and $y$ where

$$
\begin{aligned}
& x:=\sum_{i=1}^{n-1}\left(A_{i} q_{i}^{2}+B_{i} p_{i}^{2}\right), \\
& y:=B_{n} p_{n}^{2},
\end{aligned}
$$

and $A_{i}, B_{i}>0$ are constants. It satisfies $X . \psi>0$ provided that

$$
\frac{\partial \psi}{\partial x} \geq 0, \quad \frac{\partial \psi}{\partial y} \leq 0, \quad \frac{\partial \psi}{\partial x}(x, 0)>0, \quad \frac{\partial \psi}{\partial y}(y, 0)<0,
$$

and the partial derivatives are not simultaneously 0 . We can choose $\psi$ so that the level sets $\{\phi=-1\}$ and $\{\psi=1\}$ agree outside some compact set. This ensures that when we glue the handle onto our convex symplectic manifold, it still has a smooth boundary so we don't have to smooth the handle once we have attached it.

The handle $H=H_{1}^{2 n}:=\{\phi \geq-1\} \cap\{\psi \leq 1\}$. We define $\partial^{-} H$ to be the boundary $\{\phi=-1\}$. We can ensure that the only 1 -periodic orbit of $\psi$ is the critical point at the origin by [7, Section 2.2]. We wish to construct a family of 1-handles (constructed in the same way as $H)\left(H_{l}\right)_{l \in \mathbb{N}}$ with the following properties:

(1) $H_{l+1} \subset H_{l}$.

(2) The attaching region $\partial^{-} H_{l+1}$ is a subset of $\partial^{-} H_{l}$.

(3) As $l$ tends to infinity, $H_{l}$ converges uniformly to the core of the handle.

This can be done by shrinking $\psi$. Let $M$ be a compact convex symplectic manifold. After a deformation, we can assume that the boundary of $M$ has a region $A$ which is contactomorphic to the attaching region $\partial^{-} H_{1}$. We can also ensure that the period spectrum of $\partial M$ is discrete and injective (we might have to deform the region $A$ and $\phi$ slightly and hence all the handles). We can use the region $A$ to attach the handle $H_{l}$ to $M$ to create a new compact convex symplectic manifold $M_{l}:=M \cup_{\partial}-H_{l} H_{l}$. We have that $M_{l+1} \subset M_{l}$ and the boundary of each $M_{l}$ is transverse to the Liouville vector field on $M_{1}$. Let $K$ be an admissible Hamiltonian on $\widehat{M}$. We assume that $K$ has slope $S$ in a neighbourhood of $\partial M$. We choose $l$ large enough so that the attaching region $P:=\partial^{-} H_{l}$ has the property that a Reeb flowline outside $P$ intersecting $P$ twice 


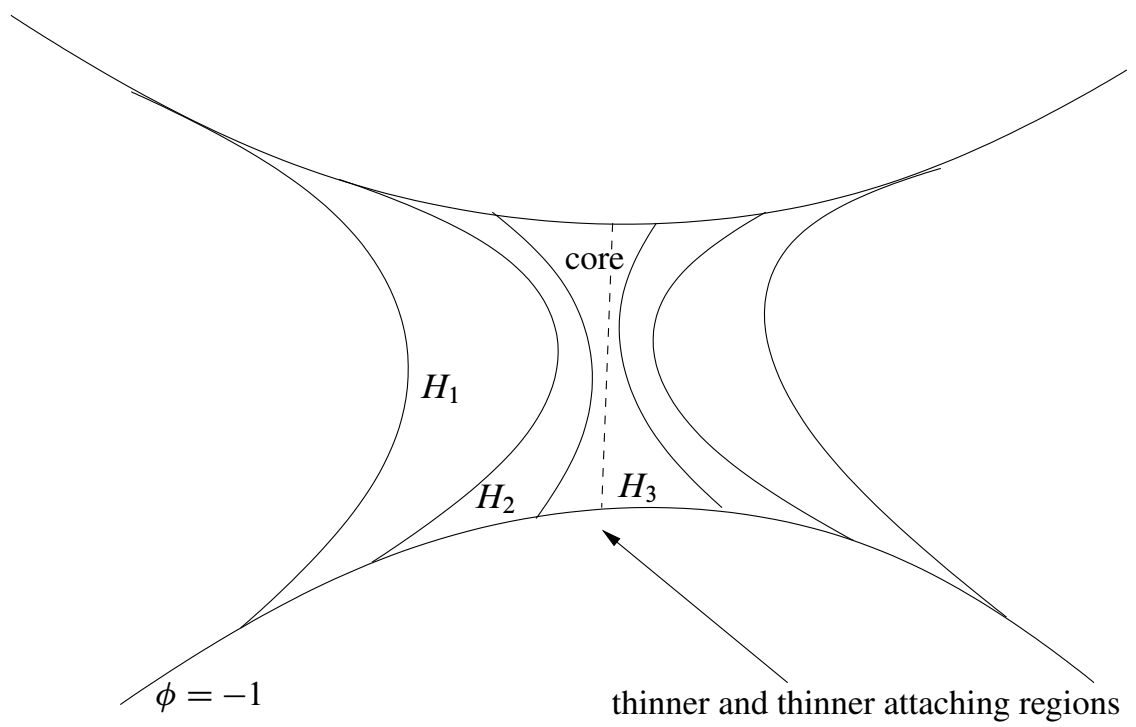

has length greater than $S$. We can now extend the Hamiltonian $K$ to a Hamiltonian $K^{\prime}: M_{l} \rightarrow \mathbb{R}$ using the function $B \psi$ where $B$ is some constant. Hence $\partial M_{l}$ is a level set of $K^{\prime}$ and $K^{\prime}$ is linearly increasing on a neighbourhood of $\partial M_{l}$. Hence we can extend $K^{\prime}$ to an admissible Hamiltonian on $\widehat{M}_{l}$. The periodic orbits of $K^{\prime}$ are the same as the periodic orbits of $K$ with an extra fixed point at the origin of the 1-handle. We can ensure that the index of the extra fixed point at the origin of the 1-handle has index strictly increasing as $S$ increases (see the last part of the proof of Theorem 1.11 in [7, Section 3.4]). Because $\partial M_{l}$ is transverse to the Liouville field of $\partial M_{1}$, we have that $\widehat{M}_{1}=\widehat{M}_{l}$ and $K^{\prime}$ is weakly admissible. Hence we have a cofinal family of weakly transfer admissible Hamiltonians $K^{\prime}$. The only orbit outside $M \subset \widehat{M_{1}}$ has arbitrarily large index, hence these $K^{\prime}$ 's induce a transfer isomorphism of rings $\mathrm{SH}_{*}(M) \rightarrow \mathrm{SH}_{*}\left(M_{1}\right)$. This proves Theorem 2.20.

\section{References}

[1] A Abbondandolo, M Schwarz, Floer homology of cotangent bundles and the loop product arXiv:0810.1995

[2] A Abbondandolo, M Schwarz, Note on Floer homology and loop space homology, from: "Morse theoretic methods in nonlinear analysis and in symplectic topology", (P Biran, O Cornea, F Lalonde, editors), NATO Sci. Ser. II Math. Phys. Chem. 217, Springer, Dordrecht (2006) 75-108 MR2276949

[3] A Abbondandolo, P Seidel, An open string analogue of Viterbo functoriality arXiv: 0712.3177 
[4] F Bourgeois, Y Eliashberg, H Hofer, K Wysocki, E Zehnder, Compactness results in symplectic field theory, Geom. Topol. 7 (2003) 799-888 MR2026549

[5] E Brieskorn, Beispiele zur Differentialtopologie von Singularitäten, Invent. Math. 2 (1966) 1-14 MR0206972

[6] A D R Choudary, A Dimca, Complex hypersurfaces diffeomorphic to affine spaces, Kodai Math. J. 17 (1994) 171-178 MR1282208

[7] K Cieliebak, Handle attaching in symplectic homology and the chord conjecture, J. Eur. Math. Soc. (JEMS) 4 (2002) 115-142 MR1911873

[8] K Cieliebak, Y Eliashberg, Symplectic geometry of Stein manifolds, in preparation

[9] K Cieliebak, A Floer, H Hofer, K Wysocki, Applications of symplectic homology. II. Stability of the action spectrum, Math. Z. 223 (1996) 27-45 MR1408861

[10] T tom Dieck, T Petrie, The Abhyankar-Moh problem in dimension 3, from: "Transformation groups (Osaka, 1987)", (K Kawakubo, editor), Lecture Notes in Math. 1375, Springer, Berlin (1989) 48-59 MR1006682

[11] T tom Dieck, T Petrie, Contractible affine surfaces of Kodaira dimension one, Japan. J. Math. (N.S.) 16 (1990) 147-169 MR1064448

[12] Y Eliashberg, Topological characterization of Stein manifolds of dimension $>2$, Internat. J. Math. 1 (1990) 29-46 MR1044658

[13] Y Eliashberg, Symplectic geometry of plurisubharmonic functions, from: "Gauge theory and symplectic geometry (Montreal, PQ, 1995)", (J Hurtubise, F Lalonde, G Sabidussi, editors), NATO Adv. Sci. Inst. Ser. C Math. Phys. Sci. 488, Kluwer Acad. Publ., Dordrecht (1997) 49-67 MR1461569 With notes by M Abreu

[14] A Floer, H Hofer, D Salamon, Transversality in elliptic Morse theory for the symplectic action, Duke Math. J. 80 (1995) 251-292 MR1360618

[15] K Fukaya, P Seidel, I Smith, Exact Lagrangian submanifolds in simply-connected cotangent bundles arXiv:math.SG/0701783

[16] R E Gompf, Handlebody construction of Stein surfaces, Ann. of Math. (2) 148 (1998) 619-693 MR1668563

[17] D Hermann, Holomorphic curves and Hamiltonian systems in an open set with restricted contact-type boundary, Duke Math. J. 103 (2000) 335-374 MR1760631

[18] S Kaliman, Exotic analytic structures and Eisenman intrinsic measures, Israel J. Math. 88 (1994) 411-423 MR1303505

[19] M McLean, The symplectic topology of Stein manifolds, $\mathrm{PhD}$ thesis, University of Cambridge (2008)

[20] A Oancea, A survey of Floer homology for manifolds with contact type boundary or symplectic homology, from: "Symplectic geometry and Floer homology. A survey of the Floer homology for manifolds with contact type boundary or symplectic homology", Ensaios Mat. 7, Soc. Brasil. Mat., Rio de Janeiro (2004) 51-91 MR2100955 
[21] A Oancea, The Künneth formula in Floer homology for manifolds with restricted contact type boundary, Math. Ann. 334 (2006) 65-89 MR2208949

[22] C P Ramanujam, A topological characterisation of the affine plane as an algebraic variety, Ann. of Math. (2) 94 (1971) 69-88 MR0286801

[23] P Seidel, Symplectic homology as Hochschild homology arXiv:math.SG/0609037

[24] P Seidel, A long exact sequence for symplectic Floer cohomology, Topology 42 (2003) 1003-1063 MR1978046

[25] P Seidel, A biased view of symplectic cohomology, from: "Current Developments in Math. (2006)”, (D Jerison, B Mazur, T Mrowka, W Schmid, R P Stanley, S-T Yau, editors), Intl. Press, Boston (2008) 211-253

[26] P Seidel, Fukaya categories and Picard-Lefschetz theory, Zürich Lect. in Adv. Math., Eur. Math. Soc., Zürich (2008) MR2441780

[27] P Seidel, I Smith, The symplectic topology of Ramanujam's surface, Comment. Math. Helv. 80 (2005) 859-881 MR2182703

[28] I Ustilovsky, Infinitely many contact structures on $S^{4 m+1}$, Internat. Math. Res. Notices (1999) 781-791 MR1704176

[29] C Viterbo, Functors and computations in Floer homology with applications. I, Geom. Funct. Anal. 9 (1999) 985-1033 MR1726235

[30] M Zaĭdenberg, Exotic algebraic structures on affine spaces, Algebra i Analiz 11 (1999) 3-73 MR1734345

Departement Mathematik, ETH Zürich

HG F 28.6, Rämistrasse 101, 8092 Zürich, Switzerland

mark.mclean@fim.math.ethz.ch

Proposed: Yasha Eliashberg

Seconded: Tom Mrowka, Leonid Polterovich
Received: 14 May 2008 Revised: 11 February 2009 